\title{
INFERÊNCIA BAYESIANA NO MODELO NORMAL ASSIMÉTRICO
}

\author{
Cristian Luis Bayes Rodríguez
}

\author{
DISSERTAÇÃO APRESENTADA AO \\ INSTITUTO DE MATEMÁTICA E ESTATÍSTICA \\ DA UNIVERSIDADE DE SÃO PAULO \\ PARA OBTENÇÃO DO TÍTULO DE \\ MESTRE EM ESTATÍSTICA
}

\author{
Área de Concentração: Estatística \\ Orientadora: Profa. Dra. Márcia D'Elia Branco \\ Durante a elaboração deste trabalho o autor \\ recebeu apoio financeiro do $C N P q$
}

São Paulo, abril de 2005 



\title{
INFERÊNCIA BAYESIANA \\ NO MODELO \\ NORMAL ASSIMÉTRICO
}

\author{
Este exemplar corresponde à redação \\ final da dissertação devidamente \\ corrigida e defendida por \\ Cristian Luis Bayes Rodríguez \\ e aprovada pela comissão julgadora.
}

São Paulo, maio de 2005.

Banca examinadora:

- Prof. Dra. Márcia D’Elia Branco (Orientadora) - IME-USP

- Prof. Dr. Heleno Bolfarine - IME-USP

- Prof. Dr. Josemar Rodrigues - UFSCar 
Aos meus pais 



\section{Agradecimentos}

- Aos meus pais, Luis e Bertila, por todo o carinho, motivação e apoio em todos os sentidos que sempre me deram.

- À minha orientadora Prof. Dra. Márcia D'Elia Branco, por sua compreensão, dedicação e orientação, que de uma maneira clara e objetiva sempre me direcionou para um bom desenvolvimento desse trabalho.

- Aos membros da banca examinadora: Prof. Dr. Heleno Bolfarine e Prof. Dr. Josemar Rodrigues, pelos seus valiosos aportes e sugestões.

- Aos companheiros da república: Christian, Jesús e Karina que se tornaram minha família aqui em São Paulo.

- Aos amigos Lourdes, Juan Carlos, Jorge, Jannet, Víctor Hugo, Vladimir, Eduardo, Thiago pelo prazer de suas amizades, conversas e trocas de conhecimentos.

- A Erika pelo apoio, preocupação e carinho nesta fase da minha vida.

- Ao Prof. Dr. Vladimir Belitsky pela sua ajuda e amizade durante o mestrado.

- Ao IME e à USP pela oportunidade de fazer meu Mestrado nesta Instituição, e ao CNPq pelo apoio financeiro.

- Aos amigos que incentivaram minha vinda para o IME-USP, como o Prof. Victor Maehara e o Prof. Jorge Chue do Departamento de Estatística - UNALM, ao Prof. Antonio Bravo e muitos outros. 
Finalmente, agradeço a Deus, por sua proteção, e por ter me dado força e perseverança ao longo de todo este período. 


\section{Resumo}

Esta dissertação compreende um estudo dos aspectos inferênciais da distribuição normal assimétrica, assim como o modelo de regressão considerando erros normais assimétricos. Nossa principal contribuição está na derivação de uma aproximação para a priori de Jeffreys e para informacão de Fisher da distribuição normal assimétrica padrão e uma proposta de uma nova distribuição a priori não subjetiva para o parâmetro de assimetria do modelo. Também propomos uma nova reparametrização, que na abordagem clássica, permite obter formas fechadas na construção do algoritmo EM, e na abordagem bayesiana, formas conhecidas para as distribuições condicionais a posteriori, o que facilita a implementação do algoritmo de Gibbs. Estimadores bayesianos como a média, a mediana e o máximo a posteriori sob as duas prioris mencionadas acima foram comparados com o estimador de máxima verossimilhança mediante um estudo de simulação. Também, foi avaliado no estudo de simulação o comportamento de estimadores intervalares, como o intervalo de confiança assintótico e os intervalos de credibilidade bayesianos. No caso de testes de hipóteses, foram comparados os desempenhos do teste de razão de verossimilhanças e do fator de Bayes. 


\section{Abstract}

This work approaches a study of the inferential aspects of the skew normal distribution, and the regression model under skew normal errors. The main contribution is to obtain a good approximation for the Jeffreys prior and the Fisher information matrix for the standard skew normal distribution, and also to propose a new non subjective prior distribution for the skewness parameter. We have also developed a new reparametrization which allow us to obtain closed forms for the EM algorithm, and from bayesian point of view, closed forms for the conditional posteriors distributions, which makes easier the implementation of the Gibbs sampling algorithm. Bayesian estimators such as a posterior mean, median and mode, under the two prior were compared with the maximum likelihood estimator through a simulation study. Also, on the simulation study the behavior of the interval estimators as the classical interval and the equal tail credibility interval and HPD was evaluated. For the hypothesis tests the behavior of the likelihood ratio test and the Bayes factor was compared. 


\section{Sumário}

1 Introdução 1

1.1 Organização da dissertação . . . . . . . . . . . . . . . . . 2

2 Distribuição Normal Assimétrica 4

2.1 Distribuição Normal Assimétrica Padrão . . . . . . . . . . . . . . . . 4

2.1.1 Funções de densidade e distribuição . . . . . . . . . . . . . . . . 4

2.1 .2 Propriedades . . . . . . . . . . . . . . . . 6

2.1 .3 Caracterizações . . . . . . . . . . . . . . . . . . . . . 12

2.2 Distribuição Normal Assimétrica de três parâmetros . . . . . . . . . . 15

$\begin{array}{llr}3 & \text { Inferência Estatística } & 18\end{array}$

3.1 Introdução . . . . . . . . . . . . . . . . . . . . . . . . 18

3.2 Inferência pelo método dos momentos . . . . . . . . . . . . . . . 19

3.3 Inferência pelo método de máxima verossimilhança . . . . . . . . 20 
3.3.1 Considerações Gerais . . . . . . . . . . . . . . . . 20

3.3 .2 Algoritmo EM . . . . . . . . . . . . . . 27

3.4 Inferência Bayesiana . . . . . . . . . . . . . . . . 30

3.4 .1 Distribuição a priori . . . . . . . . . . . . . . 30

3.4.2 O Modelo Hierárquico e o Algoritmo MCMC . . . . . . . . . . 34

3.5 Teste de Hipóteses $\ldots \ldots \ldots$. . . . . . . . . . . . . . . . . . 37

4 Aplicação 41

4.1 Aplicação aos dados de fronteira . . . . . . . . . . . . . . . . . . . . . 41

4.2 Estudo de Simulação . . . . . . . . . . . . . . . . . . . . . 43

5 Regressão Linear com erros assimétricos 48

5.1 Introdução . . . . . . . . . . . . . . . . . . . . . . . . . . 48

5.2 Inferência Clássica . . . . . . . . . . . . . . . . . . . . . . . . 49

5.3 Inferência Bayesiana . . . . . . . . . . . . . . . . . . 55

5.4 Aplicação . . . . . . . . . . . . . . . . . . . . 56

6 Considerações Finais $\quad 58$

$\begin{array}{lc}\text { A Aproximações } & 60\end{array}$

$\begin{array}{ll}\text { B Figuras } & 70\end{array}$ 
$\begin{array}{ll}\text { C Tabelas } & 72\end{array}$

$\begin{array}{lr}\text { D Programas } & 81\end{array}$

D.1 Programa em WinBUGS para o modelo de três parâmetros . . . . . . 81

D.2 Programas em $\mathrm{R} \ldots \ldots \ldots \ldots \ldots$. . . . . . . . . . 82 


\section{Lista de Figuras}

2.1 Distância $L_{1} \ldots \ldots \ldots \ldots \ldots \ldots \ldots \ldots$

3.1 Gráfico comparativo das duas especificações a priori para $\lambda \ldots$. . . . 33

3.2 Gráfico comparativo das duas especificações a priori para $\delta \ldots 33$

4.1 Histograma dos dados, distribuição estimada via kernel e ditribuições

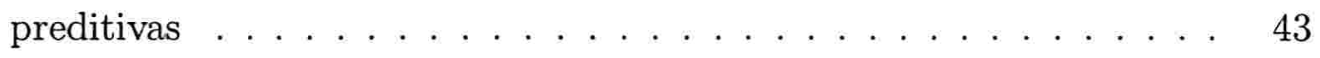

A.1 Matriz de informação de Fisher $I(\lambda)$ e sua aproximação . . . . . . . . 65

A.2 Priori de Jeffreys $f^{J}(\lambda)$ e sua aproximação . . . . . . . . . . . 66

A.3 $a_{0}(\lambda)$ e sua aproximação $\ldots \ldots \ldots \ldots \ldots 6 \ldots \ldots \ldots$

A.4 $a_{1}(\lambda)$ e sua aproximação $\ldots \ldots \ldots \ldots$. . . . . . . . 68

A.5 $M(\lambda)$ e sua aproximação . . . . . . . . . . . . . 69

B.1 Funções de densidade da normal assimétrica com valores positivos de $\lambda \quad 70$

B.2 Funções de densidade da normal assimétrica com valores negativos de $\lambda 71$ 


\section{Lista de Tabelas}

3.1 Probabilidade do e.m.v. ser infinito . . . . . . . . . . . . 21

4.1 Estimativas pontuais dos parâmetros para os dados de fronteira, sob duas diferentes especificações a priori . . . . . . . . . . . . . 42

5.1 Estimativas pontuais dos parâmetros para os dados AIS . . . . . . . . 57

C.1 Viés das estimativas no estudo de simulação . . . . . . . . . . . . 73

C.2 Erro quadrático médio das estimativas no estudo de simulação . . . . 74

C.3 Intervalo de confiança assintótico com confiança de $95 \%$. . . . . . 75

C.4 Intervalo de Credibilidade com probabilidade de $95 \% \ldots 76$

C.5 HPD com probabilidade de $95 \% \ldots \ldots \ldots \ldots$. . . . . . . 77

C.6 Porcentagem de aceitação da hipótese nula com o Teste da Razão de Verossimilhanças . . . . . . . . . . . . . . . . 78

C.7 Porcentagem de aceitação (ou rejeição) da hipótese nula $H_{0}: \lambda=0$, com o Fator de Bayes . . . . . . . . . . . . . . . . . . 79 
C.8 Porcentagem de aceitação (ou rejeição) da hipótese nula $H_{0}: \lambda=\lambda^{*}$, com o Fator de Bayes . . . . . . . . . . . . . . . . . 80 


\section{Capítulo 1}

\section{Introdução}

Em muitas situações práticas, onde a usual suposição de normalidade não é satisfeita devido a falta de simetria dos dados, propõe-se como alternativa a utilização de uma família mais geral de distribuições, de forma que consiga modelar a assimetria dos dados e além disso, incluir a distribuição normal como um caso particular. Esta família de distribuições é denominada normal assimétrica.

A distribuição normal assimétrica univariada surgiu independentemente em vários artigos estatísticos, entre os principais trabalhos pode-se destacar Roberts (1966), O'Hagan \& Leonard (1976) e Aigner, Lovell \& Schmidt (1977). Entretanto, foi Azzalini (1985) que introduziu formalmente esta distribuição, estudou suas propriedades e mostrou que a distribuição tem problemas na estimação do parâmetro que controla a assimetria, pelos métodos de estimação usuais (métodos dos momentos e de máxima verossimilhança). Métodos alternativos tem sido estudados por Sartori (2003) na abordagem clássica, e na abordagem bayesiana, por Liseo \& Loperfido (2004). Estes últimos propõem a utilização da priori de Jeffreys, entretanto esta priori tem uma expressão difícil de trabalhar. 
Generalizações para o caso multivariado dessas idéias tem sido propostas por vários autores, por exemplo, Azzalini \& Dalla Valle (1996), Azzalini \& Capitanio (1999), Sahu, Dey \& Branco (2003) e Genton, He \& Liu (2001). Estes últimos autores obtiveram os momentos de um vetor aleatório com distribuição normal assimétrica multivariada e suas formas quadráticas.

O principal objetivo deste trabalho é discutir os aspectos inferênciais na estimação dos parâmetros da distribuição normal assimétrica, para tal, propomos a abordagem bayesiana como alternativa à clássica. Através de um estudo de simulação avaliamos os desempenhos dos estimadores de máxima verossimilhança e dos estimadores bayesianos, sob duas distribuições a priori para o parâmetro de assimetria. Discutimos também a estimação por intervalos e testes de hipóteses.

Duas aplicações são consideradas. A primeira utilizando um conjunto de dados simulado por Azzalini, que é considerado um desafio em termos de estimação, já que o método de máxima verossimilhança tem um péssimo desempenho neste caso. $\mathrm{O}$ segundo, dentro de um contexto de modelos de regressão.

\subsection{Organização da dissertação}

A presente dissertação de mestrado está dividida em seis capítulos. No segundo capítulo, apresentamos definições e propriedades da distribuição normal assimétrica, onde destacamos dois resultados recentes. O primeiro apresenta uma expressão bastante útil para a função de distribuição acumulada e o segundo uma expressão para a distância $L_{1}$ entre uma distribuição normal assimétrica e uma distribuição normal.

No terceiro capítulo, apresentamos uma discusão sob a estimação dos parâmetros do modelo normal assimétrico. Primeiro, faremos uma revisão dos métodos de in- 
ferência clássica e, além disso, para a estimação por máxima verossimilhança propomos um algoritmo EM baseado em uma nova reparametrização do modelo. Na abordagem bayesiana, apresentamos uma aproximação para a priori de Jeffreys e propomos uma nova distribuição a priori para o parâmetro de assimetria do modelo; conseguimos formas conhecidas para as condicionais completas do modelo baseadas na reparametrização mencionada acima.

No quarto capítulo, apresentamos uma aplicação baseada em um conjunto de dados simulados já estudados anteriormente na literatura. Também, são apresentados os resultados de um estudo de simulação onde comparamos a inferência clássica com inferência bayesiana para o modelo normal assimétrico univariado.

No quinto capítulo, apresentamos o modelo de regressão linear simples com erros normais assimétricos, a função de escore, a matriz de informação de Fisher e a estimação dos parâmetros de regressão nas abordagens clássica e bayesiana.

Finalmente, no sexto capítulo apresentamos conclusões dos resultados obtidos neste trabalho, perspectivas futuras de trabalho. 


\section{Capítulo 2}

\section{Distribuição Normal Assimétrica}

Este capítulo apresenta uma revisão sobre as propriedades da distribuição normal assimétrica univariada. Inicialmente exploraremos o caso uniparamétrico, a qual denominaremos distribuição normal assimétrica padrão. Esta distribuição depende apenas de um parâmetro, o qual caracteriza a assimetria da sua função de densidade. Na seção 2.2.3 apresentaremos diferentes caracterizações desta distribuição. Em seguida, serão considerados também parâmetros de posição e escala.

\subsection{Distribuição Normal Assimétrica Padrão}

\subsubsection{Funções de densidade e distribuição}

Definição 2.1 Uma variável aleatória $Z$ tem distribuição normal assimétrica padrão se sua função de densidade de probabilidade é dada por

$$
f_{Z}(z)=2 \phi(z) \Phi(\lambda z) \quad(-\infty<z<\infty)
$$


onde $\phi($.$) e \Phi($.$) são as funções de densidade de probabilidade e de distribuição de uma$ normal padrão, respectivamente. O parâmetro $\lambda$ caracteriza a forma da distribuição e também é denominado parâmetro de assimetria, pois valores negativos de $\lambda$ indicam assimetria negativa e valores positivos de $\lambda$ assimetria positiva. Se $\lambda=0$ a densidade acima coincide com a densidade da distribuição normal padrão e portanto é simétrica. Utilizaremos a seguinte notação $Z \sim S N(\lambda)$. As figuras (B.1) e (B.2) no apêndice B ilustram o comportamento desta densidade para alguns valores de $\lambda$.

Proposição 2.1 A função de distribuição associada a densidade (2.1) é denotada por $F_{Z}(z ; \lambda)$ e dada por

$$
F_{Z}(z ; \lambda)=2 \Phi_{2}(z, 0 \mid \Omega), \operatorname{com} \Omega=\left[\begin{array}{cc}
1 & -\delta \\
-\delta & 1
\end{array}\right], \quad \delta=\frac{\lambda}{\sqrt{1+\lambda^{2}}} \quad(-\infty<z<\infty)
$$

sendo $\Phi_{2}(. \mid \Omega)$ a função de distribuição de uma normal bivariada com média zero e matriz de variância $\Omega$.

Através da proposição (2.1) vemos que a função de distribuição da normal assimétrica pode ser obtida facilmente se tivermos acesso a um programa que calcule a distribuição acumulada de uma normal bivariada.

Prova :

$$
F_{Z}(z ; \lambda)=2 \int_{-\infty}^{z} \phi(t) \Phi(\lambda t) d t=2 \int_{-\infty}^{z} \int_{-\infty}^{\lambda t} \phi(t) \phi(u) d u d t
$$

Fazendo a mudança de variável $v=\frac{u-\lambda t}{\sqrt{1+\lambda^{2}}}$, temos que

$$
\begin{aligned}
F_{Z}(z ; \lambda) & =2 \sqrt{1+\lambda^{2}} \int_{-\infty}^{z} \int_{-\infty}^{0} \phi(t) \phi\left(v \sqrt{1+\lambda^{2}}+\lambda t\right) d v d t \\
& =2 \int_{-\infty}^{z} \int_{-\infty}^{0} \frac{\sqrt{1+\lambda^{2}}}{2 \pi} \exp \left(-\frac{1}{2}\left[\left(1+\lambda^{2}\right) t^{2}+2 \lambda \sqrt{1+\lambda^{2}} t v+\left(1+\lambda^{2}\right) v^{2}\right]\right) d v d t
\end{aligned}
$$




$$
\begin{aligned}
& =2 \int_{-\infty}^{z} \int_{-\infty}^{0} \frac{\sqrt{1+\lambda^{2}}}{2 \pi} \exp \left(-\frac{1}{2}\left[\left[\begin{array}{l}
t \\
v
\end{array}\right]^{T}\left[\begin{array}{cc}
1+\lambda^{2} & \lambda \sqrt{1+\lambda^{2}} \\
\lambda \sqrt{1+\lambda^{2}} & 1+\lambda^{2}
\end{array}\right]\left[\begin{array}{l}
t \\
v
\end{array}\right]\right) d v d t\right. \\
& =2 \int_{-\infty}^{z} \int_{-\infty}^{0} \frac{\sqrt{1+\lambda^{2}}}{2 \pi} \exp \left(-\frac{1}{2}\left[\left[\begin{array}{l}
t \\
v
\end{array}\right]^{T}\left[\begin{array}{cc}
1 & -\frac{\lambda}{\sqrt{1+\lambda^{2}}} \\
-\frac{\lambda}{\sqrt{1+\lambda^{2}}} & 1
\end{array}\right]^{-1}\left[\begin{array}{l}
t \\
v
\end{array}\right]\right) d v d t\right. \\
& =2 \int_{-\infty}^{z} \int_{-\infty}^{0} \frac{1}{2 \pi \sqrt{1-\delta^{2}}} \exp \left(-\frac{1}{2}\left[\left[\begin{array}{l}
t \\
v
\end{array}\right]^{T}\left[\begin{array}{cc}
1 & -\delta \\
-\delta & 1
\end{array}\right]^{-1}\left[\begin{array}{l}
t \\
v
\end{array}\right]\right)\right] d v d t \\
& =2 \int_{-\infty}^{z} \int_{-\infty}^{0} \phi_{2}(t, v \mid \Omega) d v d t \\
& =2 \Phi_{2}(z, 0 \mid \Omega) .
\end{aligned}
$$

\subsubsection{Propriedades}

A densidade em (2.1) possui algumas propriedades interessantes que serão apenas listadas neste trabalho e cujas provas podem ser obtidas em Azzalini (1985) e Azzalini (2004). Entretanto, optamos por destacar algumas propriedades devido a sua importância ou novidade. Estas serão apresentadas nas proposições (2.2) e (2.3) com as respectivas provas.

Denotamos por $H N(0,1)$ a distribuição half-normal (ou normal truncada a esquerda do zero) que tem densidade dada por $2 \phi(Z) I_{[0, \infty)}(Z)$.

A distância $L_{1}$ entre duas funçòes de densidade $f_{1}$ e $f_{2}$ é definida por

$$
L_{1}\left(f_{1}, f_{2}\right)=\frac{1}{2} \int\left|f_{1}(x)-f_{2}(x)\right| d x
$$

Essa distância assume valores no intervalo $[0,1]$, quando $L_{1}\left(f_{1}, f_{2}\right)=0$ implica que $f_{1}(x)=f_{2}(x), \forall x$ e quando $L_{1}\left(f_{1}, f_{2}\right)=1$ implica a máxima discrepância. 


\section{$\underline{\text { Propriedades }}$}

1. Se $Z \sim S N(\lambda)$, então $|Z| \sim H N(0,1)$

2. Quando $\lambda \rightarrow \infty$, a densidade (2.1) converge a uma $H N(0,1)$

3. Se $Z \sim S N(\lambda)$, então $-Z \sim S N(-\lambda)$

4. A densidade (2.1) é log-concava

5. $1-F_{Z}(-z ; \lambda)=F_{Z}(z ;-\lambda)$

6. $F_{Z}(z ; 1)=\{\Phi(z)\}^{2}$

7. $\sup \left|\Phi(z)-F_{Z}(z)\right|=\pi^{-1} \arctan |\lambda|$

8. Se $Z \sim S N(\lambda)$, então $Z^{2} \sim \chi_{1}^{2}$.

Vidal, Iglesias, Branco \& Arellano-Valle (2004) calcularam a distância $L_{1}$ entre a f.d.p. de uma normal assimétrica e uma normal padrão, a qual é apresentada na seguinte proposição.

Proposição 2.2 A distância $L_{1}$ entre a f.d.p. de uma normal assimétrica dada em (2.1) e uma normal padrão é

$$
L_{1}(\lambda)=\frac{1}{2}-\frac{1}{\pi} \arccos \left(\frac{|\lambda|}{\sqrt{1+\lambda^{2}}}\right) .
$$

Prova : Utilizando a definição da distância $L_{1}$, temos

$$
L_{1}(\lambda)=\int_{-\infty}^{\infty}|\phi(z)-2 \phi(z) \Phi(\lambda z)| d z
$$




$$
\begin{aligned}
& =\int_{-\infty}^{\infty} 2\left|\frac{1}{2}-\Phi(\lambda z)\right| \phi(z) d z \\
& =\int_{-\infty}^{0}\left|\frac{1}{2}-\Phi(\lambda z)\right| 2 \phi(z) d z+\int_{0}^{\infty}\left|\frac{1}{2}-\Phi(\lambda z)\right| 2 \phi(z) d z .
\end{aligned}
$$

Agora, pela simetria da distribuição normal padrão, notamos que se $z>0$ e $\lambda>0$ ou se $z<0$ e $\lambda<0$, temos que

$$
\frac{1}{2}-\Phi(\lambda z)=-\int_{0}^{\lambda z} \phi(u) d u \text { e } \quad\left|\frac{1}{2}-\Phi(\lambda z)\right|=\int_{0}^{\lambda z} \phi(u) d u .
$$

Por outro lado, se $z<0$ e $\lambda>0$ ou se $z>0$ e $\lambda<0$, temos que

$$
\frac{1}{2}-\Phi(\lambda z)=\int_{\lambda z}^{0} \phi(u) d u=\int_{0}^{-\lambda z} \phi(u) d u
$$

Assim, se $\lambda>0$, então

$$
\begin{aligned}
L_{1}(\lambda) & =\int_{-\infty}^{0} \int_{0}^{-\lambda z} \phi(u) d u 2 \phi(z) d z+\int_{0}^{\infty} \int_{0}^{\lambda z} \phi(u) d u 2 \phi(z) d z \\
& =2 \int_{0}^{\infty} \int_{0}^{\lambda z} \phi(u) d u \phi(z) d z .
\end{aligned}
$$

Similarmente para $\lambda<0$, então

$$
L_{1}(\lambda)=2 \int_{0}^{\infty} \int_{0}^{-\lambda z} \phi(u) d u \phi(z) d z
$$

Assim, temos que

$$
L_{1}(\lambda)=2 \int_{0}^{\infty} \int_{0}^{|\lambda| z} \phi(u) d u \phi(z) d z
$$


Após algumas manipulações algébricas temos que

$$
L_{1}(\lambda)=2 \Phi(0, \Omega), \quad \text { sendo } \Omega=\left(\begin{array}{cc}
1 & |\lambda| \\
|\lambda| & 1+\lambda^{2}
\end{array}\right) \text {. }
$$

Logo utilizando um resultado bem conhecido (Fang, Kotz \& Ng 1990)

$$
2 \Phi(0, \Omega)=\frac{1}{4}+\frac{1}{2 \pi} \arcsin \left(\frac{|\lambda|}{\sqrt{1+\lambda^{2}}}\right) .
$$

temos que

$$
L_{1}(\lambda)=\frac{1}{\pi} \arcsin \left(\frac{|\lambda|}{\sqrt{1+\lambda^{2}}}\right)
$$

Desde de que $\arcsin \theta+\arccos \theta=\frac{\pi}{2}$, a distância $L_{1}$ pode ser escrita como

$$
L_{1}(\lambda)=\frac{1}{2}-\frac{1}{\pi} \arccos \left(\frac{|\lambda|}{\sqrt{1+\lambda^{2}}}\right) \text {. }
$$

$L_{1}(\lambda)$ assume como valor máximo 0.5 quando $\lambda \rightarrow \infty$, no gráfico (2.1) podemos observar o comportamento dessa função.

Figura 2.1: Distância $L_{1}$

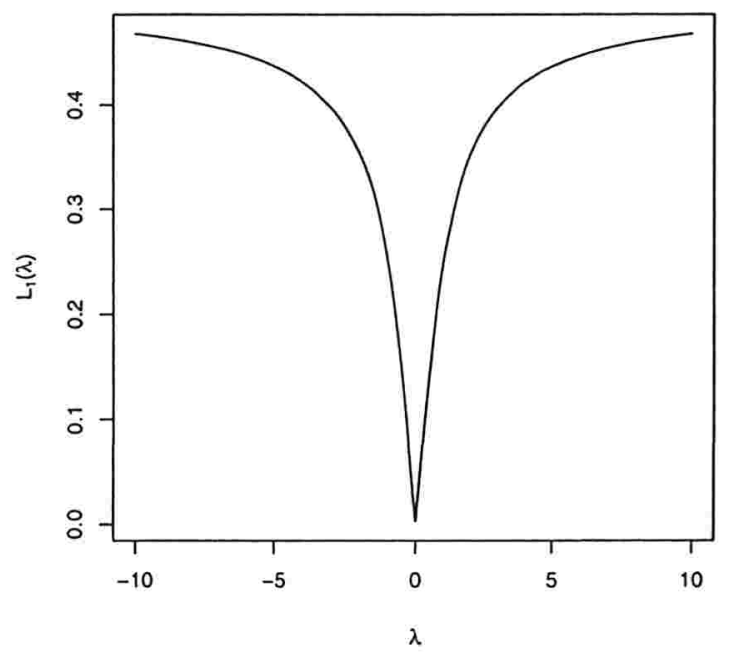


A seguir obteremos a função geradora de momentos (f.g.m) da distribuição normal assimétrica, da qual derivaremos medidas importantes para a caracterização desta distribuição, tais como, média, variância e coeficiente de assimetria. O lema 2.1 (Lachos 2004) será utilizado para a prova da proposição 2.3 .

Lema 2.1 Se $V \sim N_{k}(\eta, \Sigma)$, então $E\left[\Phi_{m}(a+A V \mid \gamma, \Gamma)\right]=\Phi_{m}\left(a \mid \gamma-A \eta, \Gamma+A \Sigma A^{T}\right)$.

Proposição 2.3 A função geradora de momentos da normal assimétrica é dada por $M_{Z}(t)=2 \exp \left(\frac{t^{2}}{2}\right) \Phi(\delta t), \operatorname{com} \delta=\frac{\lambda}{\sqrt{1+\lambda^{2}}}$.

Prova : Utilizando a definição de f.g.m., temos que

$$
\begin{aligned}
M_{Z}(t)=E\left[e^{t Z}\right] & =2 \int_{-\infty}^{\infty} e^{t z} \phi(z) \Phi(\lambda z) d z \\
& =2 \int_{-\infty}^{\infty} \frac{1}{\sqrt{2 \pi}} e^{-\frac{1}{2}\left(z^{2}-2 t z+t^{2}\right)} e^{t^{2} / 2} \Phi(\lambda z) d z \\
& =2 e^{t^{2} / 2} \int_{-\infty}^{\infty} \frac{1}{\sqrt{2 \pi}} e^{-\frac{1}{2}(z-t)^{2}} \Phi(\lambda z) d z .
\end{aligned}
$$

Fazendo a mudança de variável $x=z-t$, então,

$$
M_{Z}(t)=2 e^{t^{2} / 2} \int_{-\infty}^{\infty} \phi(x) \Phi(\lambda x+\lambda t) d x .
$$

Logo, utilizando o lema 2.1 para $k=m=1, \eta=0, \Sigma=1, a=\lambda t$ e $A=\lambda, \gamma=0$ e $\Gamma=1$ temos que

$$
\begin{aligned}
M_{Z}(t) & =2 e^{t^{2} / 2} \Phi\left(\frac{\lambda}{\sqrt{1+\lambda^{2}}} t\right) \\
& =2 e^{t^{2} / 2} \Phi(\delta t) .
\end{aligned}
$$


A partir da proposição 2.3 obtemos os momentos da normal assimétrica. Seja $Z$ uma variável aleatória com distribuição $S N(\lambda)$, temos que

$$
E(Z)=\sqrt{\frac{2}{\pi}} \frac{\lambda}{\sqrt{1+\lambda^{2}}} \text { e } \operatorname{Var}(Z)=1-\frac{2}{\pi} \frac{\lambda^{2}}{1+\lambda^{2}} .
$$

Utilizando a propriedade 8 da página 7 , segue que os momentos de ordem par de $Z$ coincidem com os da distribuição normal padrão, isto é,

$$
E\left[Z^{2 k}\right]=2^{-k} \frac{(2 k) !}{k !}
$$

Os momentos ímpares (ver Henze, 1986) são dados por

$$
E\left[Z^{2 k+1}\right]=\sqrt{\frac{2}{\pi}} \lambda\left(1+\lambda^{2}\right)^{-\left(k+\frac{1}{2}\right)} 2^{-k}[(2 k+1) !] \sum_{j=0}^{k} \frac{j !(2 \lambda)^{2 j}}{(2 j+1) !(k-j) !}
$$

Os coeficientes de assimetria e de excesso de curtose são definidos por

$$
\gamma_{1}=\frac{E\left((z-E(z))^{3}\right)}{E\left((z-E(z))^{2}\right)^{\frac{3}{2}}} \text { e } \gamma_{2}=\frac{E\left((z-E(z))^{4}\right)}{E\left((z-E(z))^{2}\right)^{2}}-3 .
$$

Utilizando (2.2) e (2.3), o coeficiente de assimetria da normal assimétrica é dado por

$$
\gamma_{1}=\sqrt{\frac{2}{\pi}}\left(\frac{4}{\pi}-1\right)\left(\frac{\lambda}{\sqrt{1+\lambda^{2}}}\right)^{3}\left(1-\frac{2}{\pi} \frac{\lambda^{2}}{1+\lambda^{2}}\right)^{-\frac{3}{2}} .
$$

Esta medida caracteriza como e quanto a distribuição se afasta da condição de simetria. $\gamma_{1}$ é uma função crescente em $|\lambda|$, e se $\lambda=0$ então $\gamma_{1}=0$. Da expressão em (2.4) obtemos que $\gamma_{1} \in[-0.99527,0.99527]$. O fato do coeficiente de assimetria ser 
limitado indica que a normal assimétrica não consegue modelar dados com grandes assimetrias.

O coeficiente de excesso de curtose é expresso por

$$
\gamma_{2}=\frac{8}{\pi^{2}}(\pi-3)\left(\frac{\lambda}{\sqrt{1+\lambda^{2}}}\right)^{4}\left(1-\frac{2}{\pi} \frac{\lambda^{2}}{1+\lambda^{2}}\right)^{-2}
$$

e é uma medida que procura caracterizar o formato da distribuição quanto ao seu achatamento, sendo menor que 0 para as platicúrticas, igual a 0 para uma distribuição mesocúrtica e maior do que 0 para as distribuições leptocúrticas. Da expressão (2.5), obtemos que este coeficiente assume valores no intervalo [0, 0.86869]. Assim, para $\lambda \neq 0$ a normal assimétrica é uma distribuição leptocúrtica e quando $|\lambda|$ cresce a curtose da distribuição aumenta e atinge seu valor máximo quando $\lambda=\infty$.

\subsubsection{Caracterizações}

A seguir apresentamos três diferentes maneiras de obter a distribuição normal assimétrica. A primeira é denominada construção por condicionamento e será apresentada na proposição 2.4 .

Proposição 2.4 Seja $Y=\left(Y_{1}, Y_{2}\right)^{T} \sim N_{2}(0, \Omega), \Omega=\left[\begin{array}{cc}1 & -\delta \\ -\delta & 1\end{array}\right]$ com $|\delta|<1 e$ definimos a variável $Z=Y_{2} \mid Y_{1}>0$. Então $Z \sim S N(\lambda)$.

Prova: Utilizando as propriedades da distribuição normal, temos que $Y_{1}, Y_{2}$ tem distribuição marginal $N(0,1)$ e que $Y_{1} \mid Y_{2}=z \sim N\left(\delta z, 1-\delta^{2}\right)$, portanto

$$
P\left(Y_{1}>0\right)=\frac{1}{2} \text { e } P\left(Y_{1}>0 \mid Y_{2}=z\right)=\Phi\left(\frac{\delta z}{\sqrt{1-\delta^{2}}}\right) \text {. }
$$


Então,

$$
\begin{aligned}
f_{Z}(z) & =\frac{P\left(Y_{1}>0 \mid Y_{2}=z\right) f_{Y_{2}}(z)}{P\left(Y_{1}>0\right)} \\
& =2 \phi(z) \Phi\left(\frac{\delta z}{\sqrt{1-\delta^{2}}}\right) .
\end{aligned}
$$

Para obter a densidade em (2.1) basta considerar a reparametrização $\lambda=\frac{\delta}{\sqrt{1-\delta^{2}}}$.

Uma segunda forma de apresentar a distribuição normal assimétrica é através da representação estocástica, à qual foi obtida por Henze (1986) e cujo resultado é dado pela proposição 2.5 definida abaixo. Esta representação será de grande utilidade para a implementação da inferência estatística no capítulo 3 .

Proposição 2.5 Sejam $Y_{1}$ e $Y_{2}$ variáveis aleatórias independentes com distribuição normal padrão, então

$$
Z=\delta\left|Y_{1}\right|+\sqrt{1-\delta^{2}} Y_{2} \sim S N(\lambda) \operatorname{com} \delta=\frac{\lambda}{\sqrt{1+\lambda^{2}}}
$$

Prova :

$$
\begin{aligned}
E\left[e^{t Z}\right] & =E\left[e^{t\left(\delta\left|Y_{1}\right|+\sqrt{1-\delta^{2}} Y_{2}\right)}\right] \\
& =E\left[e^{t \delta\left|Y_{1}\right|}\right] E\left[e^{t \sqrt{1-\delta^{2}} Y_{2}}\right] \\
& =e^{\left(1-\delta^{2}\right) \frac{t^{2}}{2}} \int_{0}^{\infty} \frac{2}{\sqrt{2 \pi}} e^{\delta t y_{1}} e^{-\frac{y_{1}^{2}}{2}} d y_{1} \\
& =2 e^{\frac{t^{2}}{2}} \int_{0}^{\infty} \frac{1}{\sqrt{2 \pi}} e^{-\frac{1}{2}\left[y_{1}^{2}-2 \delta t y_{1}+\delta^{2} t^{2}\right]} d y_{1} \\
& =2 e^{\frac{t^{2}}{2}} \int_{0}^{\infty} \frac{1}{\sqrt{2 \pi}} e^{-\frac{1}{2}\left[\left(y_{1}-\delta t\right)^{2}\right]} d y_{1} .
\end{aligned}
$$


Fazendo a mudança de variável $w=y_{1}-\delta t$, temos que

$$
\begin{aligned}
E\left[e^{t Z}\right] & =2 e^{\frac{t^{2}}{2}} \int_{-\delta t}^{\infty} \frac{1}{\sqrt{2 \pi}} e^{-\frac{w^{2}}{2}} d w \\
& =2 e^{\frac{t^{2}}{2}}(1-\Phi(-\delta t)) \\
& =2 e^{\frac{t^{2}}{2}} \Phi(\delta t) .
\end{aligned}
$$

Uma outra forma de construir a distribuição normal assimétrica é através de estatísticas de ordem, esta construção foi introduzida por Roberts (1966).

Proposição 2.6 Seja $Y=\left(Y_{1}, Y_{2}\right)^{T} \sim N_{2}(0, \Omega), \Omega=\left[\begin{array}{cc}1 & -\delta \\ -\delta & 1\end{array}\right] \operatorname{com}|\delta|<1$ então, $Z=\min \left(Y_{1}, Y_{2}\right)$ tem distribuição $S N(\lambda)$, considerando $\lambda=\frac{1-\delta}{\sqrt{1-\delta^{2}}}$.

Prova:

$$
F_{Z}(z)=F_{Y_{1}, Y_{2}}(z, z)=\int_{-\infty}^{z} \int_{-\infty}^{z} \frac{1}{2 \pi \sqrt{1-\delta^{2}}} \exp \left[-\frac{y_{1}^{2}+y_{2}^{2}-2 \delta y_{1} y_{2}}{2\left(1-\delta^{2}\right)}\right] d y_{1} d y_{2}
$$

considerando $y_{1}^{2}-2 \delta y_{1} y_{2}=\left(y_{1}-\delta y_{2}\right)^{2}-\delta y_{2}^{2}$

$$
\begin{aligned}
F_{Z}(z) & =\int_{-\infty}^{z} \frac{1}{\sqrt{2 \pi}} \exp \left[-\frac{1}{2} y_{2}^{2}\right] \int_{-\infty}^{z} \frac{1}{2 \pi \sqrt{1-\delta^{2}}} \exp \left[-\frac{\left(y_{1}-\delta y_{2}\right)^{2}}{2\left(1-\delta^{2}\right)}\right] d y_{1} d y_{2} \\
& =\int_{-\infty}^{z} \frac{1}{\sqrt{2 \pi}} \exp \left[-\frac{1}{2} y_{2}^{2}\right] \Phi\left(\frac{z-\delta y_{2}}{\sqrt{1-\delta^{2}}}\right) d y_{2} .
\end{aligned}
$$

Derivando com respeito a $z$ em ambos os lados da última expressão temos

$$
f_{Z}(z)=2 \phi(z) \Phi\left(\frac{1-\delta}{\sqrt{1-\delta^{2}}} z\right)=2 \phi(z) \Phi(\lambda z)
$$

$\operatorname{com} \lambda=\frac{1-\delta}{\sqrt{1-\delta^{2}}}$. 


\subsection{Distribuição Normal Assimétrica de três parâ- metros}

Na seguinte definição estendemos o modelo (2.1) introduzindo parâmetros de posição $(\mu \in \Re)$ e escala $(\sigma>0)$. Neste caso, utilizaremos a notação $Y \sim S N\left(\mu, \sigma^{2}, \lambda\right)$.

Definição 2.2 Uma variável aleatória $Y$ tem distribuição normal assimétrica com parâmetros de posição $\mu$ e de escala $\sigma^{2}$ se sua função de densidade de probabilidade é da forma:

$$
f_{Y}(y)=\frac{2}{\sigma} \phi\left(\frac{y-\mu}{\sigma}\right) \Phi\left(\lambda \frac{y-\mu}{\sigma}\right) \quad(-\infty<y<\infty)
$$

É fácil verificar que se $Z \sim S N(\lambda)$ e $Y=\mu+\sigma Z$, então $Y \sim S N\left(\mu, \sigma^{2}, \lambda\right)$.

Proposição 2.7 A função de distribuição de (2.7), é denotada por $F_{Y}\left(y ; \mu, \sigma^{2}, \lambda\right) e$ dada por

$\infty)$

$$
F_{Y}\left(y ; \mu, \sigma^{2}, \lambda\right)=2 \Phi_{2}\left(\frac{y-\mu}{\sigma}, 0 \mid \Omega\right), \operatorname{com} \Omega=\left[\begin{array}{cc}
1 & -\delta \\
-\delta & 1
\end{array}\right], \quad \delta=\frac{\lambda}{\sqrt{1+\lambda^{2}}}(-\infty<z<
$$

sendo $\Phi_{2}(. \mid \Omega)$ a função de distribuição de uma normal bivariada com média zero e matriz de variância $\Omega$.

Prova: $F_{Y}\left(y ; \mu, \sigma^{2}, \lambda\right)=P(Y \leq y)$ consideramos $Y=\mu+\sigma Z$ e $Z \sim S N(\lambda)$ então, $F_{Y}\left(y ; \mu, \sigma^{2}, \lambda\right)=P(\mu+\sigma Z \leq y)=P\left(Z \leq \frac{y-\mu}{\sigma}\right)=F_{Z}\left(\frac{y-\mu}{\sigma} ; \lambda\right)$.

Proposição 2.8 A função geradora de momentos da normal assimétrica é dada por

$$
M_{Y}(t)=2 e^{t \mu+\frac{t^{2} \sigma^{2}}{2}} \Phi(\delta \sigma t), \quad \text { com } \delta=\frac{\lambda}{\sqrt{1+\lambda^{2}}} .
$$


Prova :

$$
E\left(e^{t y}\right)=\int_{-\infty}^{\infty} e^{t y} \frac{2}{\sigma} \phi\left(\frac{y-\mu}{\sigma}\right) \Phi\left(\lambda \frac{y-\mu}{\sigma}\right) d y
$$

Fazendo a mudança de variável $z=\frac{y-\mu}{\sigma}$, obtemos

$$
\begin{aligned}
E\left(e^{t y}\right) & =e^{t \mu} \int_{-\infty}^{\infty} e^{t \sigma z} 2 \phi(z) \Phi(\lambda z) d z \\
& =e^{t \mu} M_{Z}(t \sigma) \\
& =e^{t \mu} 2 e^{\frac{t^{2} \sigma^{2}}{2}} \Phi(\delta \sigma t) \\
& =e^{t \mu+\frac{t^{2} \sigma^{2}}{2}} \Phi(\delta \sigma t) .
\end{aligned}
$$

A média e a variância de uma variável aleatória $Y \sim S N\left(\mu, \sigma^{2}, \lambda\right)$, são expressas por,

$$
E(Y)=\mu+\sigma \sqrt{\frac{2}{\pi}} \frac{\lambda}{\sqrt{1+\lambda^{2}}} \text { e } \operatorname{Var}(Y)=\sigma^{2}\left(1-\frac{2}{\pi} \frac{\lambda^{2}}{1+\lambda^{2}}\right)
$$

Os coeficientes de assimetria e de excesso de curtose são os mesmos do caso padrão.

Uma outra propriedade interessante, apresentada a seguir, é que qualquer combinação linear de uma variável normal assimétrica será também normal assimétrica.

Proposição 2.9 Seja $Y$ e $Y_{2}$ duas variáveis aletórias tais que $Y \sim S N\left(\mu, \sigma^{2}, \lambda\right)$ e $Y_{2}=a+b Y$, então

$$
Y_{2} \sim S N\left(a+b \mu, b^{2} \sigma^{2}, \operatorname{sinal}(b) \lambda\right) .
$$

Prova: Consideremos inicialmente o caso em que $b>0$. Assim temos que

$$
P\left(Y_{2}<r\right)=P(a+b Y<r)=P\left(Y<\frac{r-a}{b}\right)=F_{Y}\left(\frac{r-a-b \mu}{b \sigma}\right) .
$$

Então, $Y_{2} \sim S N\left(a+b \mu, b^{2} \sigma^{2}, \lambda\right)$. 
Para $b<0$,

$$
P\left(Y_{2}<r\right)=P(a+b Y<r)=P(a+b(\mu+\sigma Z)<r)=P\left(Z>\frac{r-(a-b \mu)}{b \sigma}\right)
$$

sendo $Z \sim S N(\lambda)$. Logo,

$$
P\left(Y_{2}<r\right)=1-F_{Z}\left(\frac{(a-b \mu)-r}{b \sigma}, \lambda\right) .
$$

Pela propriedade 5 da normal assimétrica padrão, temos que

$$
P\left(Y_{2}<r\right)=F_{Z}\left(\frac{r-(a-b \mu)}{b \sigma},-\lambda\right) \text {, }
$$

Então, $Y_{2} \sim S N\left(a+b \mu, b^{2} \sigma^{2},-\lambda\right)$. Finalmente, $Y_{2} \sim S N\left(a+b \mu, b^{2} \sigma^{2}, \sin a l(b) \lambda\right)$. 


\section{Capítulo 3}

\section{Inferência Estatística}

\subsection{Introdução}

Nesta seção dicutiremos diferentes métodos de inferência estatística para estimar os parâmetros do modelo normal assimétrico univariado. Métodos clássicos de inferência, como os métodos dos momentos e máxima verossimilhança apresentam alguns problemas, como por exemplo, problemas de existência no caso dos estimadores de momentos e não convergência no caso de máxima verossimilhança. Na seção 3.4 descreveremos o método bayesiano que incorpora uma distribuição a priori ao processo de modelagem, a qual, como veremos, pode ajudar a controlar os problemas com a função de verossimilhança. Na seção 3.5 apresentaremos, no contexto clássico, o teste de razão de verossimilhanças e, no contexto bayesiano, o fator de Bayes e uso do HPD para testar hipóteses sob o parâmetro de assimetria $\lambda$. 


\subsection{Inferência pelo método dos momentos}

Para o caso uniparamétrico, o estimador de momentos de $\lambda$ é a solução da seguinte equação

$$
\sqrt{\frac{2}{\pi}} \frac{\lambda}{\sqrt{1+\lambda^{2}}}=\bar{z}
$$

sendo $\bar{z}=\frac{1}{n} \sum_{i=1}^{n} z_{i}$ (média amostral), e $z=\left(z_{1}, z_{2}, \ldots, z_{n}\right)$ uma amostra aleatória de tamanho $n$ de $Z \sim S N(\lambda)$. Logo, temos que

$$
\widehat{\lambda}=\frac{\sqrt{\frac{\pi}{2}} \bar{z}}{\sqrt{1-\frac{\pi}{2} \bar{z}^{2}}},
$$

esta definido apenas para $\bar{z} \in\left[-\sqrt{\frac{2}{\pi}}, \sqrt{\frac{2}{\pi}}\right]$.

Pewsey (2000) obteve os estimadores de momentos e suas variâncias para a distribuição normal assimétrica de três parâmetros. Neste caso, consideremos $\boldsymbol{y}=$ $\left(y_{1}, y_{2}, \ldots, y_{n}\right)$ uma amostra aleatória de $Y \sim S N(\mu, \sigma, \lambda)$. Igualando os momentos amostrais aos populacionais, após diversas manipulações algébricas obtemos

$$
\begin{aligned}
& \widehat{\mu}=\bar{y}-\left(\frac{2}{4-\pi} m_{3}\right)^{\frac{1}{3}} \\
& \widehat{\sigma^{2}}=s^{2}+\left(\frac{2}{4-\pi} m_{3}\right)^{\frac{2}{3}} \\
& \widehat{\lambda}=\frac{\left(\frac{2}{4-\pi} m_{3}\right)^{\frac{1}{3}} s^{-1}}{\sqrt{\frac{2}{\pi}-\left(1-\frac{2}{\pi}\right)\left(\frac{2}{4-\pi} m_{3}\right)^{\frac{1}{3}} s^{-2}}}
\end{aligned}
$$

sendo $\bar{y}=\frac{1}{n} \sum_{i=1}^{n} y_{i}, m_{2}=s^{2}=\frac{1}{n} \sum_{i=1}^{n}\left(y_{i}-\bar{y}\right)^{2}$ e $m_{3}=\frac{1}{n} \sum_{i=1}^{n}\left(y_{i}-\bar{y}\right)^{3}$, a média amostral, o segundo e terceiro momento amostral em torno a média, respectivamente. O estimador de momentos $\hat{\lambda}$ esta definido apenas para $\frac{\left|m_{3}\right|}{s^{3}}<0.99527$. Para o caso normal, 
isto é, $\lambda=0$, o estimador de momentos de $\widehat{\sigma^{2}}$ irá superestimar o parâmetro $\sigma^{2}$ e $\widehat{\mu}$ irá superestimar o subestimar dependendo do sinal de $m_{3}$. Portanto, esses estimadores não se comportam muito bem no caso normal.

\subsection{Inferência pelo método de máxima verossimi- lhança}

\subsubsection{Considerações Gerais}

Vamos considerar inicialmente o problema de estimação do parâmetro de assimetria na distribuição normal assimétrica padrão.

Seja $\boldsymbol{z}=\left(z_{1}, z_{2}, \ldots, z_{n}\right)$ uma amostra aleatória de tamanho $n$ de $Z \sim S N(\lambda)$. Neste caso a função de verossimilhança é dada por

$$
L(\lambda ; \boldsymbol{z})=\prod_{i=1}^{n} 2 \phi\left(z_{i}\right) \Phi\left(\lambda z_{i}\right) \propto \prod_{i=1}^{n} \Phi\left(\lambda z_{i}\right)
$$

Observe que, considerando $z_{i}>0, \forall i$ a função de verossimilhança dada em (3.1) é uma função monotona crescente e portanto o estimador de máxima verossimilhança (e.m.v.) é infinito. Similarmente, se $z_{i}<0, \forall i$ o e.m.v. será menos infinito. Neste caso Liseo \& Loperfido (2004) mostraram que os casos apontados acima caracterizam totalmente as amostras cujo e.m.v. não é finito, e a probabilidade de obter-se uma amostra com e.m.v. infinito é dada por

$$
P(Z<0)^{n}+P(Z>0)^{n}=\left(\frac{1}{2}-\frac{1}{\pi} \arctan \lambda\right)^{n}+\left(\frac{1}{2}+\frac{1}{\pi} \arctan \lambda\right)^{n} .
$$

Na tabela (3.1) são apresentadas estas probabilidades para alguns valores de $n$ e $\lambda$. 
Tabela 3.1: Probabilidade do e.m.v. ser infinito

\begin{tabular}{|c|c|c|c|c|c|c|}
\hline & $\lambda=1$ & $\lambda=2$ & $\lambda=3$ & $\lambda=5$ & $\lambda=8$ & $\lambda=10$ \\
\hline $\mathrm{n}=5$ & 0.238 & 0.450 & 0.582 & 0.722 & 0.817 & 0.851 \\
$\mathrm{n}=10$ & 0.056 & 0.202 & 0.339 & 0.522 & 0.667 & 0.724 \\
$\mathrm{n}=20$ & 0.003 & 0.041 & 0.115 & 0.273 & 0.445 & 0.524 \\
$\mathrm{n}=50$ & 0.000 & 0.003 & 0.004 & 0.038 & 0.132 & 0.199 \\
$\mathrm{n}=100$ & 0.000 & 0.000 & 0.000 & 0.001 & 0.017 & 0.039 \\
\hline
\end{tabular}

Para amostras onde o estimador de máxima verossimilhança é finito, necessitamos maximizar o logaritmo da função de verossimilhança, dado por

$$
l(\lambda)=\log L(\lambda ; z)=n \log 2+\sum_{i=1}^{n} \log \phi\left(z_{i}\right)+\log \Phi\left(\lambda z_{i}\right) .
$$

Derivando (3.2) obtemos

$$
l^{\prime}(\lambda)=\sum_{i=1}^{n} z_{i} \frac{\phi\left(\lambda z_{i}\right)}{\Phi\left(\lambda z_{i}\right)}
$$

As raizes de $l^{\prime}(\lambda)$ não podem ser obtidas analiticamente, portanto, algoritmos numéricos são necessários. A segunda derivada é dada por

$$
l^{\prime \prime}(\lambda)=\sum_{i=1}^{n} \frac{\lambda z_{i}^{3} \phi\left(\lambda z_{i}\right)}{\Phi\left(\lambda z_{i}\right)}+\sum_{i=1}^{n} \frac{z_{i}^{2} \phi^{2}\left(\lambda z_{i}\right)}{\Phi^{2}\left(\lambda z_{i}\right)} .
$$

Portanto, a informação de Fisher é dada por 


$$
\begin{aligned}
I(\lambda) & =E\left[l^{\prime \prime}(\lambda)\right] \\
& =n E\left[\frac{\lambda z^{3} \phi(\lambda z)}{\Phi(\lambda z)}+\frac{z^{2} \phi^{2}(\lambda z)}{\Phi^{2}(\lambda z)}\right] \\
& =n E\left[\frac{z^{2} \phi^{2}(\lambda z)}{\Phi^{2}(\lambda z)}\right] \\
& =n \int_{-\infty}^{\infty} 2 z^{2} \phi(z) \frac{\phi^{2}(\lambda z)}{\Phi(\lambda z)} d z
\end{aligned}
$$

resultando em

$$
I(\lambda)=n \int_{-\infty}^{\infty} 2 z^{2} \phi(z) \frac{\phi^{2}(\lambda z)}{\Phi(\lambda z)} d z
$$

Recentemente Sartori (2003) propos um estimador alternativo ao e.m.v. de $\lambda$ baseado numa correção de viés dada por Firth (1993). Este estimador sempre é finito e será denotado por $\tilde{\lambda}$. Para obtermos o estimador $\tilde{\lambda}$ devemos solucionar a equação

$$
l^{\prime}(\lambda)+M(\lambda)=0
$$

sendo

$$
M(\lambda)=-\frac{\lambda}{2} \frac{a_{4}(\lambda)}{a_{2}(\lambda)}
$$

com $a_{k}=E_{Z}\left[z^{k}\left(\frac{\phi(\lambda z)}{\Phi(\lambda z)}\right)^{2}\right], k=0,1,2, \ldots$, onde os valores esperados são avaliados na distribuição normal assimétrica padrão e é necessário utilizar métodos numéricos.

No apêndice A apresentaremos uma aproximação numérica para estas esperanças a qual facilitará a obtenção do estimador corrigido de Sartori $\widetilde{\lambda}$.

Vamos discutir agora o caso mais geral da distribuição normal assimétrica com três parâmetros. Seja $\boldsymbol{y}=\left(y_{1}, y_{2}, \ldots, y_{n}\right)$ uma amostra aleatória de tamanho $n$ de 
$Y \sim S N(\mu, \sigma, \lambda)$, a função de verossimilhança é dada por

$$
L(\mu, \sigma, \lambda ; \boldsymbol{y})=\prod_{i=1}^{n} \frac{2}{\sigma} \phi\left(\frac{y_{i}-\mu}{\sigma}\right) \Phi\left(\lambda \frac{y_{i}-\mu}{\sigma}\right) .
$$

sendo $\phi($.$) e \Phi($.$) as funções de densidade de probabilidade e de distribuição de uma$ normal padrão, respectivamente. Aplicando o logaritmo natural em ambos os lados da equação (3.6) temos que

$$
l(\mu, \sigma, \lambda)=\log L(\mu, \sigma, \lambda ; \boldsymbol{y})=n \log 2-n \log \sigma+\frac{1}{2 \sigma^{2}} \sum_{i=1}^{n}\left(y_{i}-\mu\right)^{2}+\sum_{i=1}^{n} \log \Phi\left(\lambda \frac{y_{i}-\mu}{\sigma}\right) .
$$

As equações de verossimilhança são obtidas derivando-se $l(\mu, \sigma, \lambda)$ em relação a cada parâmetro e igualando o resultado a zero. Assim temos as seguintes equacões

$$
\begin{aligned}
& \frac{\partial l}{\partial \mu}=\frac{1}{\sigma^{2}} \sum_{i=1}^{n}\left(y_{i}-\mu\right)-\frac{\lambda}{\sigma} \sum_{i=1}^{n} \frac{\phi\left(\lambda \frac{y_{i}-\mu}{\sigma}\right)}{\Phi\left(\lambda \frac{y_{i}-\mu}{\sigma}\right)}=0 \\
& \frac{\partial l}{\partial \sigma}=-\frac{n}{\sigma}+\frac{1}{\sigma^{3}} \sum_{i=1}^{n}\left(y_{i}-\mu\right)^{2}-\frac{\lambda}{\sigma^{2}} \sum_{i=1}^{n}\left(y_{i}-\mu\right) \frac{\phi\left(\lambda \frac{y_{i}-\mu}{\sigma}\right)}{\Phi\left(\lambda \frac{y_{i}-\mu}{\sigma}\right)}=0 \\
& \frac{\partial l}{\partial \lambda}=\frac{1}{\sigma} \sum_{i=1}^{n}\left(y_{i}-\mu\right) \frac{\phi\left(\lambda \frac{y_{i}-\mu}{\sigma}\right)}{\Phi\left(\lambda \frac{y_{i}-\mu}{\sigma}\right)}=0
\end{aligned}
$$

As segundas derivadas são dadas a seguir,

$$
\begin{aligned}
\frac{\partial^{2} l}{\partial \mu^{2}} & =-\frac{n}{\sigma^{2}}-\frac{\lambda^{2}}{\sigma^{2}} \sum_{i=1}^{n} \lambda \frac{y_{i}-\mu}{\sigma} \frac{\phi\left(\lambda \frac{y_{i}-\mu}{\sigma}\right)}{\Phi\left(\lambda \frac{y_{i}-\mu}{\sigma}\right)}+\sum_{i=1}^{n} \lambda \frac{y_{i}-\mu}{\sigma} \phi^{2}\left(\lambda \frac{y_{i}-\mu}{\sigma}\right) \Phi^{2}\left(\lambda \frac{y_{i}-\mu}{\sigma}\right) \\
\frac{\partial^{2} l}{\partial \sigma^{2}} & =\frac{n}{\sigma^{2}}-\frac{3}{\sigma} \sum_{i=1}^{n}\left(\frac{y_{i}-\mu}{\sigma}\right)^{2}-\frac{2 \lambda}{\sigma^{2}} \sum_{i=1}^{n}\left(\frac{y_{i}-\mu}{\sigma}\right) \frac{\phi\left(\lambda \frac{y_{i}-\mu}{\sigma}\right)}{\Phi\left(\lambda \frac{y_{i}-\mu}{\sigma}\right)} \\
& +\sum_{i=1}^{n} \frac{\lambda^{3}}{\sigma}\left(\frac{y_{i}-\mu}{\sigma}\right)^{3} \frac{\phi\left(\lambda \frac{y_{i}-\mu}{\sigma}\right)}{\Phi\left(\lambda \frac{y_{i}-\mu}{\sigma}\right)}+\sum_{i=1}^{n} \frac{\lambda^{2}}{\sigma^{2}}\left(\frac{y_{i}-\mu}{\sigma}\right)^{2} \frac{\phi^{2}\left(\lambda \frac{y_{i}-\mu}{\sigma}\right)}{\Phi^{2}\left(\lambda \frac{y_{i}-\mu}{\sigma}\right)} .
\end{aligned}
$$




$$
\begin{aligned}
\frac{\partial^{2} l}{\partial \lambda^{2}} & =-\sum_{i=1}^{n} \lambda\left(\frac{y_{i}-\mu}{\sigma}\right)^{3} \frac{\phi\left(\lambda \frac{y_{i}-\mu}{\sigma}\right)}{\Phi\left(\lambda \frac{y_{i}-\mu}{\sigma}\right)}+\sum_{i=1}^{n}\left(\frac{y_{i}-\mu}{\sigma}\right)^{2} \frac{\phi^{2}\left(\lambda \frac{y_{i}-\mu}{\sigma}\right)}{\Phi^{2}\left(\lambda \frac{y_{i}-\mu}{\sigma}\right)} \\
\frac{\partial^{2} l}{\partial \mu \partial \sigma} & =-\frac{2}{\sigma^{3}} \sum_{i=1}^{n}\left(y_{i}-\mu\right)-\frac{\lambda^{3}}{\sigma^{2}} \sum_{i=1}^{n}\left(\frac{y_{i}-\mu}{\sigma}\right)^{2} \frac{\phi\left(\lambda \frac{y_{i}-\mu}{\sigma}\right)}{\Phi\left(\lambda \frac{y_{i}-\mu}{\sigma}\right)} \\
& +\frac{\lambda^{2}}{\sigma^{2}} \sum_{i=1}^{n} \frac{y_{i}-\mu}{\sigma} \frac{\phi^{2}\left(\lambda \frac{y_{i}-\mu}{\sigma}\right)}{\Phi^{2}\left(\lambda \frac{y_{i}-\mu}{\sigma}\right)}+\frac{\lambda}{\sigma^{2}} \sum_{i=1}^{n} \frac{\phi\left(\lambda \frac{y_{i}-\mu}{\sigma}\right)}{\Phi\left(\lambda \frac{y_{i}-\mu}{\sigma}\right)} \\
\frac{\partial^{2} l}{\partial \mu \partial \lambda} & =-\frac{\lambda^{2}}{\sigma} \sum_{i=1}^{n}\left(\frac{y_{i}-\mu}{\sigma}\right)^{2} \frac{\phi\left(\lambda \frac{y_{i}-\mu}{\sigma}\right)}{\Phi\left(\lambda \frac{y_{i}-\mu}{\sigma}\right)}-\frac{\lambda}{\sigma} \sum_{i=1}^{n} \frac{y_{i}-\mu}{\sigma} \frac{\phi^{2}\left(\lambda \frac{y_{i}-\mu}{\sigma}\right)}{\Phi^{2}\left(\lambda \frac{y_{i}-\mu}{\sigma}\right)} \\
& -\frac{1}{\sigma} \sum_{i=1}^{n} \frac{\phi\left(\lambda \frac{y_{i}-\mu}{\sigma}\right)}{\Phi\left(\lambda \frac{y_{i}-\mu}{\sigma}\right)} \\
\frac{\partial^{2} l}{\partial \sigma \partial \lambda} & =-\frac{1}{\sigma} \sum_{i=1}^{n}\left(\frac{y_{i}-\mu}{\sigma}\right) \frac{\phi\left(\lambda \frac{y_{i}-\mu}{\sigma}\right)}{\Phi\left(\lambda \frac{y_{i}-\mu}{\sigma}\right)}+\lambda^{2} \sum_{i=1}^{n}\left(\frac{y_{i}-\mu}{\sigma}\right)^{3} \frac{\phi\left(\lambda \frac{y_{i}-\mu}{\sigma}\right)}{\Phi\left(\lambda \frac{y_{i}-\mu}{\sigma}\right)} \\
& +\frac{\lambda}{\sigma} \sum_{i=1}^{n}\left(\frac{y_{i}-\mu}{\sigma}\right)^{2} \frac{\phi^{2}\left(\lambda \frac{y_{i}-\mu}{\sigma}\right)}{\Phi^{2}\left(\lambda \frac{y_{i}-\mu}{\sigma}\right)} .
\end{aligned}
$$

Soluções analíticas ou explícitas das equações de verossimilhança não podem ser obtidas para os parâmetros da distribuição normal assimétrica. Observamos que o ponto $(\mu, \sigma, \lambda)=\left(\bar{y}, \sqrt{\frac{1}{n} \sum_{i=1}^{n}\left(y_{i}-\bar{y}\right)^{2}}, 0\right)$ é uma raiz das equações de verossimilhança. Mas, se avaliamos esse ponto na matriz das $2^{\underline{a}}$ derivadas o resultado é 


$$
\left[\begin{array}{ccc}
-\frac{n}{\frac{1}{n} \sum_{i=1}^{n}\left(y_{i}-\bar{y}\right)^{2}} & 0 & -\frac{\sqrt{\frac{2}{\pi}} n}{\sqrt{\frac{1}{n} \sum_{i=1}^{n}\left(y_{i}-\bar{y}\right)^{2}}} \\
0 & -\frac{2 n}{\frac{1}{n} \sum_{i=1}^{n}\left(y_{i}-\bar{y}\right)^{2}} & 0 \\
-\frac{\sqrt{\frac{2}{\pi} n}}{\sqrt{\frac{1}{n} \sum_{i=1}^{n}\left(y_{i}-\bar{y}\right)^{2}}} & 0 & \frac{2}{\pi} n
\end{array}\right]
$$

que é uma matriz indefinida, então $\left(\bar{y}, \sqrt{\frac{1}{n} \sum_{i=1}^{n}\left(y_{i}-\bar{y}\right)^{2}}, 0\right)$ é um ponto de sela da função de verossimilhança.

A matriz de informação de Fisher é representada por

$$
\begin{aligned}
& I_{F}(\mu, \sigma, \lambda)=\left[\begin{array}{ccc}
\frac{1+\lambda^{2} a_{0}}{\sigma^{2}} & \frac{\sqrt{2 / \pi} \lambda\left(1+2 \lambda^{2}\right)\left(1+\lambda^{2}\right)^{-\frac{3}{2}}+\lambda^{2} a_{1}}{\sigma^{2}} & \frac{\sqrt{2 / \pi}\left(1+\lambda^{2}\right)^{-\frac{3}{2}}-\lambda a_{1}}{\sigma} \\
\frac{\sqrt{2 / \pi} \lambda\left(1+2 \lambda^{2}\right)\left(1+\lambda^{2}\right)^{-\frac{3}{2}}+\lambda^{2} a_{1}}{\sigma^{2}} & \frac{2+\lambda^{2} a_{2}}{\sigma^{2}} & -\frac{\lambda a_{2}}{\sigma} \\
\frac{\sqrt{2 / \pi}\left(1+\lambda^{2}\right)^{-\frac{3}{2}}-\lambda a_{1}}{\sigma} & -\frac{\lambda a_{2}}{\sigma} & a_{2}
\end{array}\right] \\
& \operatorname{com} a_{k}=E_{Z}\left[Z^{k}\left(\frac{\phi(\lambda Z)}{\Phi(\lambda Z)}\right)^{2}\right], \quad k=0,1,2 .
\end{aligned}
$$

Se avaliamos a matriz de informação de Fisher em $\lambda=0$, obtemos

$$
I_{F}(\mu, \sigma, \lambda=0)=\left[\begin{array}{ccc}
\frac{1}{\sigma^{2}} & 0 & \sqrt{\frac{2}{\pi}} \frac{1}{\sigma} \\
0 & \frac{2}{\sigma^{2}} & 0 \\
\sqrt{\frac{2}{\pi}} \frac{1}{\sigma} & 0 & \frac{2}{\pi}
\end{array}\right]
$$


cujo determinante é zero, então, a matriz de informação de Fisher é singular para $\lambda=0$.

Assim, ao considerarmos o modelo de três parâmetros, temos os seguintes problemas:

1. o e.m.v. para $\lambda$ pode ser infinito,

2. a informação de Fisher é singular quando $\lambda=0$,

3. temos um ponto de sela em $(\mu, \sigma, \lambda)=\left(\bar{y}, \sqrt{\frac{1}{n} \sum_{i=1}^{n}\left(y_{i}-\bar{y}\right)^{2}}, 0\right)$

O problema de singularidade da matriz de informação de Fisher acontece em geral quando a média do modelo está superparametrizada, como é o caso da normal assimétrica onde $E(Y)=\mu+\sigma \frac{2}{\pi} \frac{\lambda}{\sqrt{1+\lambda^{2}}}$ depende dos três parâmetros. Para resolver este problema, Azzalini (1985) sugere a seguinte reparametrização do modelo,

$$
\begin{aligned}
& \varsigma=\mu+\sigma \frac{2}{\pi} \frac{\lambda}{\sqrt{1+\lambda^{2}}} \\
& \omega=\sigma \frac{2}{\pi} \frac{\lambda^{2}}{1+\lambda^{2}}
\end{aligned}
$$

e $\gamma_{1}$ o coeficiente de assimetria do modelo dado em (2.4), para uma maior discussão ver Pewsey (2000) e Azzalini (2004).

Para o caso do modelo de três parâmetros, Sartori (2003) sugere utilizar um método de estimação em dois passos. No primeiro passo, calculamos os e.m.v. de $\mu \mathrm{e}$ $\sigma$. Então, consideramos $\mu$ e $\sigma$ fixos e iguais aos e.m.v., o método de Firth é aplicado somente ao parâmetro de forma $\lambda$. 


\subsubsection{Algoritmo EM}

A maximização direta da verossimilhança em (3.6) pode apresentar problemas computacionais, além disso, este método parece não ser robusto com respeito aos valores iniciais, isto é, se não são utilizados valores iniciais adequados, o método de aproximação direta pode não convergir. A seguir apresentamos o algoritmo EM para calcular o e.m.v. para a verossimilhança do modelo de três parâmetros.

Utilizaremos, uma reparametrização do modelo que nos permite obter formas fechadas na maximização do logaritmo da função de verossimilhança aumentada (Passo M), o que difere da rotina sn.em do pacote sn do $\mathrm{R}$ dada por Azzalini, que necessita de uma maximização numérica.

Utilizando a representação estocástica de Henze (1986) dada por (2.6), podemos reescrever o modelo de três parâmetros, como

$$
Y_{i}=\mu+\sigma\left(\frac{\lambda}{\sqrt{1+\lambda^{2}}} U_{i}+\frac{1}{\sqrt{1+\lambda^{2}}} V_{i}\right)
$$

com $U_{i} \sim H N(0,1)$ e $V_{i} \sim N(0,1)$. Considerando a seguinte reparametrização

$$
\tau=\sigma \frac{\lambda}{\sqrt{1+\lambda^{2}}} \text { e } \eta=\sigma \frac{1}{\sqrt{1+\lambda^{2}}}
$$

temos que,

$$
Y_{i}=\mu+\eta U_{i}+\tau V_{i}
$$

e condicionando em $U_{i}$, obtemos a seguinte forma hieráquica para o modelo normal assimétrico

$$
\begin{aligned}
Y_{i} \mid U_{i} & \sim N\left(\mu+\eta U_{i}, \tau^{2}\right) \\
U_{i} & \sim H N(0,1) .
\end{aligned}
$$


Consideramos as variáveis aleatórias $U_{i}, \quad i=1, \ldots, n$ como variáveis latentes (não observáveis). $\boldsymbol{y}=\left(y_{1}, y_{2}, \ldots, y_{n}\right)$ denota os dados observados e $\theta=(\mu, \tau, \eta)$ o novo vetor de parâmetros, temos que o logaritmo da função de verossimilhança aumentada associada a $\left(y_{i}, U_{i}\right), \quad i=1, \ldots, n$, pode ser escrito como

$$
l_{c}(\theta)=n \log a-\frac{1}{2 \tau^{2}} \sum_{i=1}^{n}\left(y_{i}-\mu-\eta U_{i}\right)^{2}-\frac{1}{2} \sum_{i=1}^{n} U_{i}^{2} .
$$

A seguir calculamos a distribuição condicional de $U \mid y, \theta$, (para simplificar a notação omite-se o índice $i$ )

$$
\begin{aligned}
f(U \mid y, \theta) & \propto \frac{1}{\tau} \exp \left[-\frac{1}{2 \tau^{2}}(y-\mu-\eta U)^{2}\right] \exp \left[-\frac{1}{2} U^{2}\right] I_{[0, \infty)}(U) \\
& \propto \exp \left[-\frac{1}{2 \frac{\tau^{2}}{\tau^{2}+\eta^{2}}}\left(U-(y-\mu) \frac{\eta}{\tau^{2}+\eta^{2}}\right)^{2}\right] I_{[0, \infty)}(U)
\end{aligned}
$$

Obtemos que $U \mid y, \theta$ segue uma distribuição normal truncada especificada a seguir

$$
U \mid y, \theta \sim N\left((y-\mu) \frac{\eta}{\tau^{2}+\eta^{2}}, \frac{\tau^{2}}{\tau^{2}+\eta^{2}}\right) I_{[0, \infty)}(U) .
$$

Das propriedades da distribuição normal truncada Johnson, Kotz \& Balakrishnan (1994), temos que

$$
\begin{gathered}
E(U \mid \theta, y)=(y-\mu) \frac{\eta}{\tau^{2}+\eta^{2}}+\frac{\tau}{\sqrt{\tau^{2}+\eta^{2}}} \frac{\phi\left(\frac{\eta}{\tau} \frac{y-\mu}{\sqrt{\tau^{2}+\eta^{2}}}\right)}{\Phi\left(\frac{\eta}{\tau} \frac{y-\mu}{\sqrt{\tau^{2}+\eta^{2}}}\right)} \\
E\left(U^{2} \mid \theta, y\right)=(y-\mu)^{2} \frac{\eta^{2}}{\left(\tau^{2}+\eta^{2}\right)^{2}}+\frac{\tau^{2}}{\tau^{2}+\eta^{2}}(y-\mu) \frac{\tau \eta}{\left(\tau^{2}+\eta^{2}\right)^{\frac{3}{2}}} \frac{\phi\left(\frac{\eta}{\tau} \frac{y-\mu}{\sqrt{\tau^{2}+\eta^{2}}}\right)}{\Phi\left(\frac{\eta}{\tau} \frac{y-\mu}{\sqrt{\tau^{2}+\eta^{2}}}\right)}
\end{gathered}
$$

Denotando por $\widehat{U}=E(U \mid \theta=\widehat{\theta}, Y=y)$ e $\widehat{U^{2}}=E\left(U^{2} \mid \theta=\widehat{\theta}, Y=y\right)$, 
a esperança do logaritmo da verossimilhanca aumentada é expressa por

$$
\begin{aligned}
Q(\theta, \widehat{\theta})=E\left(l_{c}(\theta) \mid y, \widehat{\theta}\right)= & n \log \tau-\frac{1}{2 \tau^{2}}\left[\sum_{i=1}^{n}\left(y_{i}-\mu\right)^{2}-2 \eta \sum_{i=1}^{n}\left(y_{i}-\mu\right) \widehat{U}_{i}+\eta^{2} \sum_{i=1}^{n} \widehat{U_{i}^{2}}\right] \\
& -\frac{1}{2} \sum_{i=1}^{n} \widehat{U_{i}^{2}} .
\end{aligned}
$$

As primeiras derivadas com respeito aos parâmetros, são

$$
\begin{aligned}
& \frac{\partial Q}{\partial \mu}=\frac{1}{2 \tau^{2}}\left[2 \sum_{i=1}^{n}\left(y_{i}-\mu\right)-2 \eta \sum_{i=1}^{n} \widehat{U}_{i}\right] \\
& \frac{\partial Q}{\partial \eta}=\frac{1}{2 \tau^{2}}\left[2 \sum_{i=1}^{n}\left(y_{i}-\mu\right) \widehat{U}_{i}-2 \eta \sum_{i=1}^{n} \widehat{U_{i}^{2}}\right] \\
& \frac{\partial Q}{\partial \tau}=-\frac{n}{\tau}+\frac{1}{\tau^{3}}\left[\sum_{i=1}^{n}\left(y_{i}-\mu\right)^{2}-2 \eta \sum_{i=1}^{n}\left(y_{i}-\mu\right) \widehat{U}_{i}+\eta^{2} \sum_{i=1}^{n} \widehat{U_{i}^{2}}\right] .
\end{aligned}
$$

Igualando a zero as expressões acima, o ponto que maximiza $Q(\theta, \widehat{\theta})$ é dado por

$$
\begin{aligned}
& \widehat{\eta}=\frac{\sum_{i=1}^{n} y_{i} \widehat{U}_{i}-n \bar{y} \bar{U}}{\sum_{i=1}^{n} \widehat{U_{i}^{2}}-n \bar{U}} \\
& \widehat{\mu}=\bar{y}-\widehat{\eta} \bar{U} \\
& \widehat{\tau}=\left(\frac{1}{n}\left[\sum_{i=1}^{n}\left(y_{i}-\widehat{\mu}\right)^{2}-2 \widehat{\eta} \sum_{i=1}^{n}\left(y_{i}-\widehat{\mu}\right) \widehat{U}_{i}+\widehat{\eta}^{2} \sum_{i=1}^{n} \widehat{U_{i}^{2}}\right]\right)^{\frac{1}{2}}
\end{aligned}
$$

$\operatorname{com} \bar{U}=\frac{\sum_{i=1}^{n} \widehat{U}_{i}}{n}$. Assim obtemos o seguinte algoritmo EM:

Passo E: Dado $\theta=\widehat{\theta}$, calcular $\widehat{U}_{i}$ e $\widehat{U_{i}^{2}}, i=1, \ldots, n$, utilizando (3.7),

Passo M: Atualizar $\widehat{\theta}$, maximizando (3.8) $\operatorname{sob} \theta$, utilizando (3.9). 


\subsection{Inferência Bayesiana}

Sob a abordagem bayesiana, além de definirmos um modelo probabilístico para os dados condicional a um vetor de parâmetros, devemos também definir um modelo probabilístico para os parâmetros. Assim, a modelagem completa deverá considerar dois modelos probabilísticos, neste caso, $Y \mid \theta \sim S N(\mu, \sigma, \lambda)$ e $f(\theta)$ alguma densidade de probabilidade em $\Re \times \Re^{+} \times \Re$, denominada distribuição a priori, onde $\theta=(\mu, \sigma, \lambda)$.

Seja $\boldsymbol{y}=\left(y_{1}, \ldots, y_{n}\right)$ uma amostra aleatória simples de $Y \mid \theta$, a inferência a respeito de $\theta$ será baseada na distribuição a posteriori, obtida via fórmula de Bayes por

$$
f(\theta \mid \boldsymbol{y})=\frac{f(\theta) L(\theta ; \boldsymbol{y})}{\int f(\theta) L(\theta ; \boldsymbol{y}) d \theta}
$$

\subsubsection{Distribuição a priori}

Uma questão importante é a escolha da distribuição a priori. Em princípio, toda inferência bayesiana basea-se no conceito subjetivo de probabilidade, ver Paulino, Turkman \& Murteira (2003). Uma priori subjetiva para $\lambda$ deverá descrever a nossa incerteza a respeito deste parâmetro, baseando-se no nosso conhecimento prévio a respeito do problema. Por exemplo, uma densidade a priori com massa concentrada em valores positivos de $\lambda$ indicaria que acreditamos que a assimetria dos dados deverá ser positiva. Assim, o estabelecimento de uma priori subjetiva depende do particular problema que estamos tratando e deverá ser discutida caso a caso.

No entanto, se quizermos nos furtar da responsabilidade da escolha de uma distribuição a priori subjetiva, poderiamos, por exemplo, considerar uma priori vaga, isto é, uma densidade com grande dispersão que no caso limite resultaria em $f(\lambda) \propto$ Constante. Tal priori é denominada priori imprópria pois não integra um e portanto não é uma densidade de probabilidade. O uso de prioris impróprias deve ser feito 
com muito cuidado para garantir que não irão gerar posterioris impróprias, o que seria incoerente do ponto de vista da inferência bayesiana.

Inicialmente iremos discutir a escolha da distribuição a priori para o parâmetro de assimetria $\lambda$ no caso uniparamétrico. Uma possibilidade é utilizar a priori de Jeffreys para $\lambda$, a qual é dada por

$$
\begin{aligned}
f^{J}(\lambda) & \propto \sqrt{I(\lambda)} \\
& \propto \sqrt{\int_{-\infty}^{\infty} 2 z^{2} \phi(z) \frac{\phi^{2}(\lambda z)}{\Phi(\lambda z)} d z .}
\end{aligned}
$$

Liseo \& Loperfido (2004) obtiveram as seguintes propriedades:

- $f^{J}(\lambda)$ é simétrica em torno de $\lambda=0$ e decrescente em $|\lambda|$,

- a cauda de $f^{J}(\lambda)$ é da ordem $O\left(\lambda^{-\frac{3}{2}}\right)$.

Estas propriedades garantem que a densidade a priori de Jeffreys é própria. Podemos observar que é difícil trabalhar com a expressão (3.11), razão pela qual, estamos propondo neste trabalho a seguinte aproximação para a priori de Jeffreys (3.11)

$$
f^{J}(\lambda) \approx t\left(0, \frac{\pi^{2}}{4}, \frac{1}{2}\right)
$$

É importante notar que a cauda desta aproximação tem a mesma ordem da priori de Jeffreys, isto é, $O\left(\lambda^{-\frac{3}{2}}\right)$.

Para obter o resultado em (3.12), utilizamos a seguinte aproximação de funções obtida por Chaibub Neto \& Branco (2003),

$$
\frac{1}{\pi} \frac{\phi(z)}{\sqrt{\Phi(z)[1-\Phi(z)]}} \approx \frac{1}{\sqrt{2 \pi}(\pi / 2)} \exp \left(-\frac{z^{2}}{2\left(\pi^{2} / 4\right)}\right) .
$$


Além disso, observamos que

$$
\begin{aligned}
I(\lambda) & =\int_{-\infty}^{\infty} 2 z^{2} \phi(z) \frac{\phi^{2}(\lambda z)}{\Phi(\lambda z)} d z \\
& =\int_{0}^{\infty} 2 z^{2} \phi(z) \frac{\phi^{2}(\lambda z)}{\Phi(\lambda z)} d z+\int_{-\infty}^{0} 2 z^{2} \phi(z) \frac{\phi^{2}(\lambda z)}{\Phi(\lambda z)} d z \\
& =\int_{0}^{\infty} 2 z^{2} \phi(z) \frac{\phi^{2}(\lambda z)}{\Phi(\lambda z)} d z+\int_{0}^{\infty} 2 z^{2} \phi(z) \frac{\phi^{2}(\lambda z)}{1-\Phi(\lambda z)} d z \\
& =\int_{0}^{\infty} 2 z^{2} \phi(z) \frac{\phi^{2}(\lambda z)}{\Phi(\lambda z)[1-\Phi(\lambda z)]} d z .
\end{aligned}
$$

Logo, substituindo (3.13) na expresão acima, temos que

$$
\begin{aligned}
I(\lambda) & \approx \int_{0}^{\infty} 2 z^{2} \phi(z) \frac{\pi}{2} \frac{1}{\left(\pi^{2} / 4\right)} \exp \left(-\frac{\lambda^{2} z^{2}}{\left(\pi^{2} / 4\right)}\right) d z \\
& =\frac{\pi}{\left(\pi^{2} / 4\right)}\left(1+\frac{2 \lambda^{2}}{\left(\pi^{2} / 4\right)}\right)^{-1 / 2} \int_{0}^{\infty} z^{2} \phi\left(z \mid 0,\left(1+\frac{2 \lambda^{2}}{\left(\pi^{2} / 4\right)}\right)^{-1}\right) d z \\
& =\frac{2}{\pi}\left(1+\frac{2 \lambda^{2}}{\left(\pi^{2} / 4\right)}\right)^{-\frac{3}{2}}
\end{aligned}
$$

Então,

$$
f^{J}(\lambda) \approx \sqrt{\frac{2}{\pi}}\left(1+\frac{2 \lambda^{2}}{\left(\pi^{2} / 4\right)}\right)^{-\frac{3}{4}}
$$

Uma outra possibilidade de estabelecermos uma priori objetiva e própria é considerarmos a parametrização $\delta=\frac{\lambda}{\sqrt{1+\lambda^{2}}}$, o qual, é limitado no intervalo $[-1,1]$. Neste caso a escolha natural seria $\delta \sim U(-1,1)$, uma uniforme no intervalo $[-1,1]$, a qual induz no espaço paramétrico de $\lambda$ uma distribuição t-Student com os parâmetros especificados a seguir

$$
\lambda \sim t\left(0, \frac{1}{2}, 2\right) .
$$


Figura 3.1: Gráfico comparativo das duas especificações a priori para $\lambda$

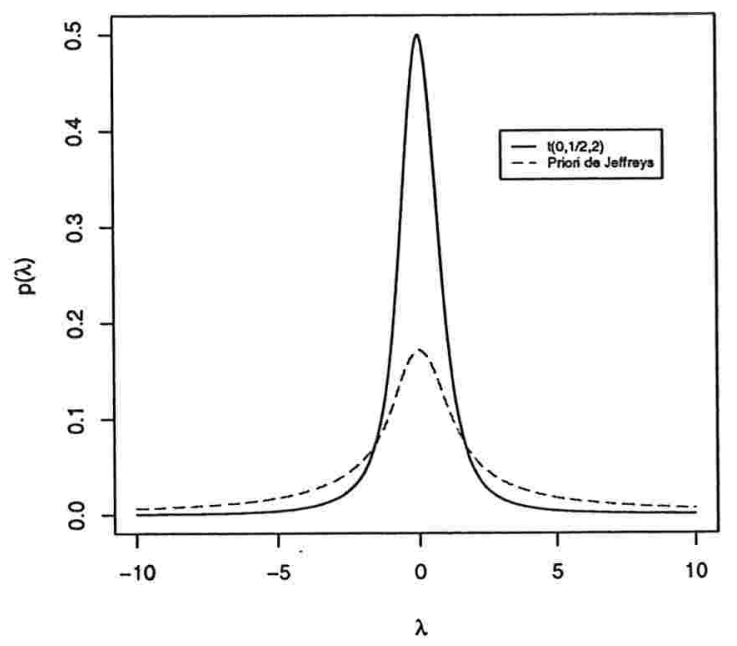

Na figura 3.1 podemos observar que a priori de Jeffreys é menos informativa do que a $t$-Student induzida pela uniforme. Se consideramos o parâmetro $\delta$ a priori de Jeffreys terá um formato de U, o qual é mostrado na figura 3.2.

Figura 3.2: Gráfico comparativo das duas especificações a priori para $\delta$

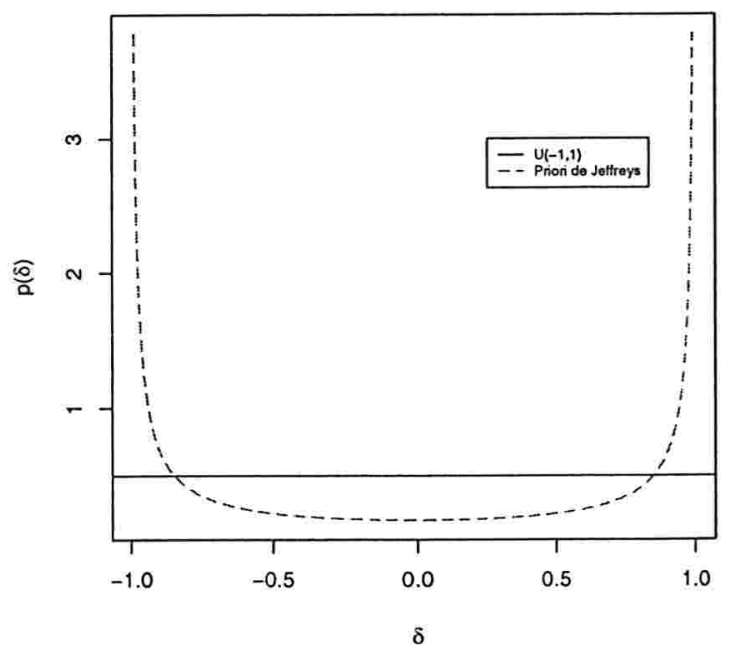


Se considerarmos o caso de três parâmetros, Liseo \& Loperfido (2004) utilizando o método de Berger \& Bernardo (1992), obtiveram a seguinte distribuição a priori de referência

$$
f(\lambda, \mu, \sigma) \propto \frac{1}{\sigma} g(\lambda)
$$

Onde $g(\lambda)$ é uma função complicada de $\lambda$ mas é integrável e tem caudas da ordem $O\left(\lambda^{-\frac{3}{2}}\right)$, razão pela qual, os autores sugerem utilizarmos por simplicidade, a priori de Jeffreys obtida para o caso uniparamétrico no lugar de $g(\lambda)$.

Lembramos que a priori de Jeffreys para os parâmetros de posição e escala é dada por $\frac{1}{\sigma}$. Em nossa análise, vamos utilizar a seguinte especificação a priori

$$
p(\lambda, \mu, \sigma) \propto \frac{1}{\sigma} p(\lambda)
$$

onde $\lambda \sim t\left(0, \sigma_{t}^{2}, k\right)$. Quando $k=\frac{1}{2}$ e $\sigma_{t}^{2}=\frac{\pi^{2}}{4}$ obtemos a aproximação da priori de Jeffreys e para $k=2$ e $\sigma_{t}^{2}=\frac{1}{2}$ obtevemos a priori induzida pela uniforme, $\delta \sim$ $U(-1,1)$. Então,

$$
p(\lambda, \mu, \sigma) \propto \frac{1}{\sigma}\left(1+\frac{\lambda^{2}}{k \sigma_{t}^{2}}\right)^{-\frac{k+1}{2}} .
$$

\subsubsection{O Modelo Hierárquico e o Algoritmo MCMC}

Consideremos $z=\left(z_{1}, z_{2}, \ldots, z_{n}\right)$ uma amostra aleatória de tamanho $n$ de $Z \sim S N(\lambda)$ e a seguinte especificação a priori, $\lambda \sim t\left(0, \sigma_{t}^{2}, k\right)$. Quando $k=\frac{1}{2}$ e $\sigma_{t}^{2}=\frac{\pi^{2}}{4}$ obtemos a aproximação da priori de Jeffreys e para $k=2$ e $\sigma_{t}^{2}=\frac{1}{2}$ obtemos a priori induzida pela uniforme, $\delta \sim U(-1,1)$. Então, utilizando a fórmula de Bayes dada em (3.10) a distribuição a posteriori é dada por 


$$
f(\lambda \mid z) \propto\left(1+\frac{\lambda^{2}}{k \sigma_{t}^{2}}\right)^{-\frac{k+1}{2}} \prod_{i=1}^{n} \Phi\left(\lambda z_{i}\right) .
$$

Utilizando a expressão dada em (3.19) podemos calcular, por métodos numéricos, quantidades de interesse como a média, a mediana e o máximo a posteriori, assim como intervalos de credibilidade com caudas iguais e HPD para $\lambda$.

Uma forma hierárquica de reescrever este modelo é utilizando a representação estocástica de Henze (1986), desta forma temos que

$$
Z_{i}=\frac{\lambda}{\sqrt{1+\lambda^{2}}} U_{i}+\frac{1}{\sqrt{1+\lambda^{2}}} V_{i}
$$

onde $U_{i} \sim H N(0,1)$ e $V_{i} \sim N(0,1)$ são variáveis aleatórias independentes. Então condicionando em $U_{i}$ temos que

$$
\begin{aligned}
Z_{i} \mid U_{i} & \sim N\left(\frac{\lambda}{\sqrt{1+\lambda^{2}}} U_{i}, \frac{1}{1+\lambda^{2}}\right), \\
U_{i} & \sim H N(0,1) .
\end{aligned}
$$

A distribuição a priori $\lambda \sim t\left(0, \sigma_{t}^{2}, k\right)$, também pode ser reescrita em forma hierárquica da seguinte maneira

$$
\begin{aligned}
\lambda \mid w & \sim N\left(0, \frac{\sigma_{t}^{2}}{w}\right) \\
w & \sim \text { Gamma }\left(\frac{k}{2}, \frac{k}{2}\right) .
\end{aligned}
$$

A representação dada em (3.21) e a especificação a priori dada em (3.22) são convenientes para implementar o amostrador de Gibbs no programa WinBUGS.

Ao considerarmos o caso de três parâmetros, como no caso uniparamétrico utilizaremos a representação estocástica para reescrever o modelo. Neste caso,

$$
y_{i}=\mu+\sigma\left(\frac{\lambda}{\sqrt{1+\lambda^{2}}} U_{i}+\frac{1}{\sqrt{1+\lambda^{2}}} V_{i}\right)
$$


onde $U_{i} \sim H N(0,1)$ e $V_{i} \sim N(0,1)$ são variáveis aleatórias independentes. Logo, condicionando en $U_{i}$ obtemos

$$
\begin{aligned}
Y_{i} \mid U_{i} & \sim N\left(\mu+\sigma \delta U_{i}, \sigma^{2}\left(1-\delta^{2}\right)\right) \\
U_{i} & \sim H N(0,1) .
\end{aligned}
$$

Para facilitar o trabalho computacional consideraremos a seguinte reparametrização $\eta=\sigma \delta$ e $\tau=\sigma \sqrt{1-\delta^{2}}$, então

$$
\begin{aligned}
Y_{i} \mid U_{i} & \sim N\left(\mu+\eta U_{i}, \tau^{2}\right) \\
U_{i} & \sim H N(0,1) .
\end{aligned}
$$

A função de verossimilhança aumentada na nova parametrização é dada por

$$
L(\mu, \tau, \eta)=\frac{1}{\tau^{n}} \exp \left(-\frac{1}{2 \tau^{2}} \sum_{i=1}^{n}\left(y_{i}-\mu-\eta u_{i}\right)^{2}\right) \exp \left(-\frac{1}{2} \sum_{i=1}^{n} u_{i}^{2}\right) \prod_{i=1}^{n} I_{[0, \infty)}\left(u_{i}\right) .
$$

Utilizando-se (3.22), a priori proposta em (3.18) pode ser reescrita como

$$
f(\lambda, \mu, \sigma, w) \propto \frac{1}{\sigma^{2}} \exp \left(-\frac{1}{2} \frac{\lambda^{2} w}{\sigma_{t}^{2}}\right) w^{\frac{k+1}{2}-1} \exp \left(-\frac{k}{2} w\right)
$$

a qual induz a seguinte priori na nova reparametrização,

$$
f(\mu, \tau, \eta, w) \propto \frac{1}{\tau^{2}} \exp \left(-\frac{1}{2} \frac{\eta^{2} w}{\tau^{2} \sigma_{t}^{2}}\right) w^{\frac{k+1}{2}-1} \exp \left(-\frac{k}{2} w\right) .
$$

Finalmente, a distribuição a posteriori aumentada é dada por

$$
f(\mu, \tau, \eta, w \mid D) \propto f(\mu, \tau, \eta, w) L(\mu, \tau, \eta) .
$$

Resultando em formas conhecidas para todas as distribuições condicionais a posteri- 
ori. As condicionais completas são representadas pelas seguintes expressões:

$$
\begin{aligned}
& w \mid \mu, \tau, \eta, U, Y \sim \operatorname{Gamma}\left(\frac{k+1}{2}, \frac{1}{2}\left(\frac{\eta^{2}}{\sigma_{t}^{2} \tau^{2}}+k\right)\right) \\
& u_{i} \mid \mu, \tau, \eta, w, Y \sim N\left(\frac{\left(y_{i}-\mu\right) \eta}{\tau^{2}+\eta^{2}}, \frac{\tau^{2}}{\tau^{2}+\eta^{2}}\right) I_{[0, \infty)}\left(u_{i}\right) \forall i, i=1, \ldots, n \\
& \mu \mid \tau, \eta, w, U, Y \sim N\left(\frac{\sum_{i=1}^{n}\left(y_{i}-\eta u_{i}\right)}{n}, \frac{\tau^{2}}{n}\right) \\
& \eta \mid \mu, \tau, w, U, Y \sim N\left(\frac{\sum_{i=1}^{n}\left(y_{i}-\mu\right) u_{i}}{\frac{w}{\sigma_{t}^{2}}+\sum_{i=1}^{n} u_{i}^{2}}, \frac{\tau^{2}}{\frac{w}{\sigma_{t}^{2}}+\sum_{i=1}^{n} u_{i}^{2}}\right) \\
& \frac{1}{\tau^{2}} \mid \mu, \eta, w, U, Y \sim G a m m a\left(\frac{n+1}{2}, \frac{1}{2}\left(\frac{w \eta^{2}}{\sigma_{t}^{2}}+\sum_{i=1}^{n}\left(y_{i}-\mu-\eta u_{i}\right)^{2}\right)\right)
\end{aligned}
$$

Considerando (3.30) podemos utilizar o amostrador de Gibbs para a inferência bayesiana sob os parâmetros do modelo e implementar nosso próprio programa, como foi feito neste trabalho e é apresentado no apêndice D. No caso de preferirmos utilizar o WinBUGS, podemos utilizar a representação hierárquica do modelo dada em (3.24) e a especificação a priori expressa em (3.27).

\subsection{Teste de Hipóteses}

Vamos a considerar aqui apenas teste de hipóteses bilaterais. Sob o ponto de vista clássico, vamos considerar o teste de razão de verossimilhanças (RV), e sob a abor- 
dagem bayesiana o uso do fator de Bayes e o uso do HPD.

Se desejamos verificar se um conjunto de dados $\boldsymbol{z}=\left(z_{1}, z_{2}, \ldots, z_{n}\right)$ apresentam evidências significativas para o uso da normal assimétrica, no lugar da usual suposição de normalidade, podemos testar as hipóteses $H_{0}: \lambda=0$ vs $H_{1}: \lambda \neq 0$. Denotando por $\widehat{\lambda}$ o e.m.v. de $\lambda$ e após algumas manipulações algébricas, a estatística do teste de RV é expressa por

$$
\xi_{R V}=2\left[\sum_{i=1}^{n} \log \Phi\left(\widehat{\lambda} z_{i}\right)+n \log 2\right] .
$$

No caso de uma hipótese mais geral como $H_{0}: \lambda=\lambda^{*}$ vs $H_{1}: \lambda \neq \lambda^{*}$, para $\lambda^{*}$ fixado, temos que a forma desta estatística é dada por

$$
\xi_{R V}=2\left[\sum_{i=1}^{n} \log \Phi\left(\widehat{\lambda} z_{i}\right)-\sum_{i=1}^{n} \log \Phi\left(\lambda^{*} z_{i}\right)\right] .
$$

Assintoticamente e sob a hipótese nula, tem-se que $\xi_{R V} \sim \chi_{1}^{2}$. Também devemos ter em conta que este teste pode ser aplicado apenas nos casos em que o e.m.v. seja finito.

No caso bayesiano, se consideramos uma distribuição a priori própria podemos utilizar o fator de Bayes para comparar a hipótese $H_{0}: \lambda=\lambda^{*}$ vs $H_{1}: \lambda \neq \lambda^{*}$. Liseo \& Loperfido (2004) utilizaram esta metodologia assumindo a priori de Jeffreys dada em (3.11) e Vidal et al. (2004) assumiram como priori para $\lambda^{2}$ uma distribuição gama. Nós utilizaremos o fator de Bayes considerando como priori $\lambda \sim t\left(0, \sigma_{t}^{2}, k\right)$ que para $k=1 / 2$ e $\sigma_{t}^{2}=\frac{\pi^{2}}{4}$ é uma aproximação para a priori de Jeffreys.

Utilizaremos a seguinte notação $\operatorname{Gamma}(. \mid a, b)$ para representar uma distribuição gama com parâmetro de forma $a$ e de escala $b$.

Considerando $\lambda \sim t\left(0, \sigma_{t}^{2}, k\right)$ como distribuição a priori, a expressão para o fator 
de Bayes em favor da hipótese nula é dada por

$$
B F=\frac{\prod_{i=1}^{n} \Phi\left(\lambda^{*} z_{i}\right)}{\int_{-\infty}^{\infty} t\left(\lambda \mid 0, \sigma_{t}^{2}, k\right) \prod_{i=1}^{n} \Phi\left(\lambda z_{i}\right) d \lambda}
$$

Logo, utilizando a representação hierárquica da distribuição t-Student dada em (3.22), podemos reescrever a expressão acima como

$$
B F=\frac{\prod_{i=1}^{n} \Phi\left(\lambda^{*} z_{i}\right)}{\int_{-\infty}^{\infty} \int_{0}^{\infty} \phi\left(\lambda \mid 0, \frac{\sigma_{t}^{2}}{w}\right) \prod_{i=1}^{n} \Phi\left(\lambda z_{i}\right) \operatorname{Gamma}\left(w \mid \frac{k}{2}, \frac{k}{2}\right) d w d \lambda}
$$

Mudando a ordem de integração, temos que

$$
B F=\frac{\prod_{i=1}^{n} \Phi\left(\lambda^{*} z_{i}\right)}{\int_{0}^{\infty} E_{S}\left[\Phi_{n}(\lambda z)\right] \operatorname{Gamma}\left(w \mid \frac{k}{2}, \frac{k}{2}\right) d w}
$$

onde $S \sim \operatorname{Normal}\left(0, \frac{\sigma_{t}^{2}}{w}\right)$ e $\Phi_{n}($.$) é a funcão de distribuição de uma normal multivaria-$ da com média zero e matriz de variância-covariância $I_{n}$. Finalmente, utilizando o lema 2.1 temos que

$$
B F=\frac{\prod_{i=1}^{n} \Phi\left(\lambda^{*} z_{i}\right)}{\int_{0}^{\infty} \Phi_{n}\left(0 \mid I_{n}+\frac{\sigma_{t}^{2}}{w} z z^{T}\right) \operatorname{Gamma}\left(w \mid \frac{k}{2}, \frac{k}{2}\right) d w} .
$$

No caso particular em que $H_{0}: \lambda=0$ vs $H_{1}: \lambda \neq 0$ o fator de Bayes em favor da hipótese de normalidade é dado por

$$
B F_{0}=\frac{1}{2^{n} \int_{0}^{\infty} \Phi_{n}\left(0 \mid I_{n}+\frac{\sigma_{t}^{2}}{w} z z^{T}\right) \operatorname{Gamma}\left(w \mid \frac{k}{2}, \frac{k}{2}\right) d w}
$$


Uma outra possibilidade para testar as hipóteses $H_{0}: \lambda=\lambda^{*}$ vs $H_{1}: \lambda \neq \lambda^{*}$ é calcular o HPD de tamanho $1-\alpha$, e verificar se $\lambda^{*}$ está em essa região ou não. Para calcular o HPD utilizamos métodos numéricos, minimizando a seguinte função (Chen, Shao \& Ibrahim 2001):

$$
\left|f\left(\lambda_{s} \mid z\right)-f\left(\lambda_{i} \mid z\right)\right|+\left|F\left(\lambda_{s} \mid z\right)-F\left(\lambda_{i} \mid z\right)-(1-\alpha)\right|
$$

onde $\lambda_{s}>\lambda_{i}$. 


\section{Capítulo 4}

\section{Aplicação}

\subsection{Aplicação aos dados de fronteira}

Nesta seção utilizaremos os dados de fronteira apresentados por Azzalini em sua página web http : //azzalini.stat.unipd.it/SN/, que consistem de uma amostra de 50 observações de uma $S N(0,1,5)$. Estes dados são interessantes pois o e.m.v. para o parâmetro de assimetria $\lambda$ é infinito, embora pelo histograma (ver figura 4.1) dos dados não pareça que a distribuição half normal $(\lambda=\infty)$ seja a mais adequada para ajustá-los. Sartori (2003) obteve um valor de estimativa mais adequado, $\tilde{\lambda}=9.14$.

Na Tabela 4.1 apresentamos as estimativas de máxima verossimilhança (e.m.v.), a média, a mediana e o máximo a posteriori, considerando as duas prioris para $\lambda$ especificadas na seção 3.4.1., para os parâmetros do modelo.

Verifica-se que as estimativas de $\mu$ e $\sigma$ não apresentam muita diferença entre si e em relação aos verdadeiros valores dos parâmetros. No entanto, para $\lambda$ essas estimativas diferem muito. Para este parâmetro, a mediana a posteriori sob a priori 
II apresentou o melhor resultado. Sob a priori I o melhor resultado foi obtido pelo máximo a posteriori. Também podemos observar a assimetria positiva da distribuição a posteriori, e no caso da priori de Jeffreys uma cauda pesada a direita.

Tabela 4.1: Estimativas pontuais dos parâmetros para os dados de fronteira, sob duas diferentes especificações a priori

\begin{tabular}{|c|c|c|c|c|c|c|c|}
\hline & & \multicolumn{3}{|c|}{ Priori I: Jeffreys } & \multicolumn{3}{c|}{ Priori II: Uniforme } \\
\hline Param & e.m.v. & Máximo & Média & Mediana & Máximo & Média & Mediana \\
\hline$\lambda$ & $\infty$ & 6.53 & $\infty$ & 31.27 & 3.82 & 7.61 & 5.26 \\
$\mu$ & -0.11 & -0.03 & -0.10 & -0.11 & 0.03 & 0.06 & -0.02 \\
$\sigma^{2}$ & 1.51 & 1.31 & 1.61 & 1.58 & 1.18 & 1.30 & 1.27 \\
\hline
\end{tabular}

Para o cálculo do máximo a posteriori foram utilizados algoritmos de maximização numérica fornecidos pela rotina optim do programa $\mathrm{R}$, os valores da média e da mediana a posteriori foram obtidos através do amostrador de Gibbs. Para todas as simulações foram geradas 1000000 amostras com burn-in de 100000 para garantir a convergência. No entanto, devido a alta autocorrelação entre as cadeias, tomamos valores de 200 em 200 para obtermos uma amostra de Monte Carlo de tamanho 5000 aproximadamente independente.

A Figura 4.1 confirma os resultados apresentados na tabela 4.1, pode-se observar que a distribuição preditiva sob priori uniforme ajusta melhor aos dados. 
Figura 4.1: Histograma dos dados, distribuição estimada via kernel e ditribuições preditivas

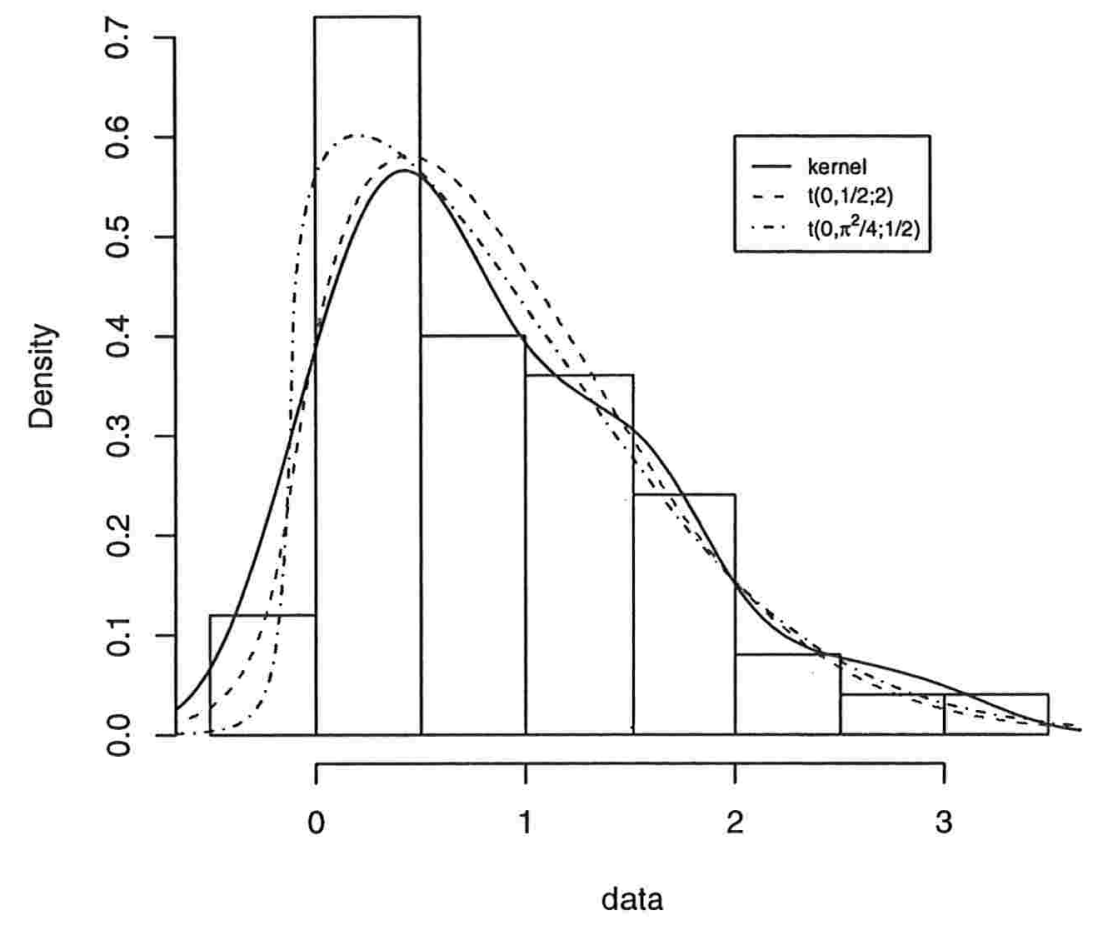

\subsection{Estudo de Simulação}

Para o nosso estudo de simulação consideramos o caso uniparamétrico, sendo que para cada valor de $\lambda=(0,0.1,0.25,0.3,0.5,0.75,1,1.5,2,3,5,8,10)$ foram geradas 10000 amostras de tamanho $n$ de uma $S N(\lambda)$, com $n=30,50,100,200$. Para cada conjunto de dados calculamos o e.m.v., a média, a mediana e o máximo a posteriori sob as duas distribuições a priori apresentadas anteriormente, assim como intervalos de credibilidade com caudas iguais e HPD de probabilidade 95\%. Para estimar a média a posteriori, utilizamos um método de integração numérica por quadratura 
adaptativa fornecido pela rotina integrate do $\mathrm{R}$, para o caso da mediana posteriori foi implementado um método de Newton-Raphson expressa da seguinte forma

$$
M e^{i+1}=M e^{i}+\frac{F(\lambda \mid D)-0.5}{f(\lambda \mid D)}
$$

sendo $f(\lambda \mid D)$ e $F(\lambda \mid D)$ as funções de densidade de probabilidade a posteriori e de distribuição acumulada a posteriori, respectivamente, as quais foram avaliadas numericamente. Para o cálculo do máximo a posteriori utilizou-se a rotina "optim"do R com o método "L-BFGS-B"(Quase-Newton). No cálculo dos intervalos de credibilidade simples também utilizamos o método de Newton-Raphson de forma similar ao utilizado no cálculo da mediana. Para o cálculo do HPD minimizamos a função dada em (3.31) através da rotina optim do R.

No presente estudo de simulação avaliamos o viés e o erro quadrático médio empíricos para o parâmetro $\lambda$. O viés empírico é definido como

$$
\operatorname{Vies}(\lambda)=\frac{\sum_{i=1}^{n}(\lambda-\tilde{\lambda})}{n},
$$

onde $\widetilde{\lambda}$ é uma estimativa de $\lambda$. Analogamente o erro quadrático médio empírico é definido por

$$
\operatorname{Eqm}(\lambda)=\frac{\sum_{i=1}^{n}(\lambda-\tilde{\lambda})^{2}}{n}
$$

Nas tabelas (C.1) e (C.2) apresentamos o viés e os erros quadráticos médios das estimativas, respectivamente. Como discutido anteriormente, o e.m.v. e a média a posteriori sob priori de Jeffreys podem ser infinitos, estes casos foram desconsiderados no cálculo do viés e o Eqm. As últimas colunas das tabelas (C.1) e (C.2) apresentam a porcentagem de vezes que nos obtivemos valores finitos para estas estimativas, isto é, a porcentagem de amostras consideradas para os cálculos das colunas 3 e 5 das tabelas (C.1) e (C.2). 
Em relação ao viés, podemos observar que, na maioria dos casos, a média a posteriori sob priori uniforme e o máximo a posteriori sob priori de Jeffreys apresentaram melhores resultados que os outros estimadores. O mesmo ocorre quando observamos o erro quadrático médio. Notou-se também que a utilização da priori uniforme pode nos levar a sub-estimar o verdadeiro valor do parâmetro para tamanhos de amostra pequenos $(n<30)$. Nestes casos todas as estimativas não apresentaram um bom comportamento, o que nos levou a não apresentá-las aqui. Essa observação sugere que estimar a assimetria para amostras muito pequenas pode não ser adequado.

Nas tabelas (C.3), (C.4) e (C.5) para cada combinação de $\lambda$ e $n$, sob as duas especificações a priori, apresentamos os seguintes resultados

- média dos limites inferiores dos intervalos (LI),

- média dos limites superiores dos intervalos (LS),

- a porcentagem de vezes que os intervalos contém o valor zero $(0 \in I)$,

- a porcentagem de vezes que os intervalos contém o verdadeiro valor do parâmetro $(\lambda \in I)$,

Em (C.3) consideramos o intervalo de confiança assintótico, em (C.4) e (C.5) os intervalos de credibilidade de caudas iguais e o HPD, respectivamente.

Na estimação intervalar, observamos que uma porcentagem alta dos intervalos de confiança assintóticos para grandes valores $\lambda$ e tamanhos de amostra pequenos (por exemplo, ver tabela C.3 para $\lambda>3$ e $n=30$ ) contém o valor zero. Esse problema não ocorre com o uso dos intervalos de credibilidade, como mostram as tabelas (C.4) e (C.5).

Em geral a medida que o valor de $\lambda$ aumenta os intervalos de credibilidade sob a 
priori de Jeffreys tem uma amplitude muito grande. A priori uniforme reduz substancialmente a amplitude dos intervalos a posteriori, sem comprometer a sua confiança. Além disso, para estes intervalos na grande maioria dos casos o verdadeiro valor do parâmetro está contido no intervalo pelo menos $90 \%$ das vezes.

Os resultados da simulação para o teste da razão de verossimilhanças encontramse na tabela (C.6), para cada combinação de $n$ e $\lambda$ temos a probabilidade empírica de aceitar a hipótese nula para cada uma das hipóteses propostas. Observa-se que o teste de razão de verossimilhanças não consegue detectar a assimetria dos dados para tamanhos de amostra e valores de $\lambda$ pequenos (por exemplo, ver a tabela (C.6) para $n=30$ e $\lambda<0.3)$. No entanto, obtém melhores resultados para testar a hipótese $H_{0}: \lambda=\lambda^{*}$ vs $H_{1}: \lambda \neq \lambda^{*}$.

Para o caso do fator de Bayes, consideramos como forte evidência a favor da hipótese nula se $B F>2$, e a favor da hipótese alternativa se $B F<0.5$. Na tabela (C.7) para a hipótese $H_{0}: \lambda=0$ vs $H_{1}: \lambda \neq 0$, são apresentados os seguintes resultados relativos ao fator de Bayes, o qual denotaremos por $B F_{0}$,

- a porcentagem de vezes que $B F_{0}>2$ sob a priori de Jeffreys,

- a porcentagem de vezes que $B F_{0}<0.5$ sob a priori de Jeffreys,

- a porcentagem de vezes que $B F_{0}>2$ sob a priori uniforme,

- a porcentagem de vezes que $B F_{0}<0.5$ sob a priori uniforme.

Na tabela (C.8) são apresentados os resultados da simulação relativos ao fator de Bayes para a hipótese $H_{0}: \lambda=\lambda^{*}$ vs $H_{1}: \lambda \neq \lambda^{*}$, de forma similar ao descrito acima.

Pela tabela (C.7) notamos que em geral o fator de Bayes baseado na distribuição 
a priori uniforme tende a detectar melhor a assimetria dos dados. Para valores de $\lambda$ maiores do que 1 , a porcentagem de vezes que $B F_{0}>2$ é praticamente igual a zero.

Na tabela (C.8), observamos que o fator de Bayes da priori de Jeffreys para a hipótese $H_{0}: \lambda=\lambda^{*}$ vs $H_{1}: \lambda \neq \lambda^{*}$, obtém melhores resultados que o da priori uniforme.

Finalmente, considerando que:

- Em relação a estimação pontual do parâmetro $\lambda$, em geral a média a posteriori sob priori uniforme apresenta os melhores resultados em relação ao viés e o erro quadrático médio.

- Na estimação intervalar, observamos que os intervalos de credibilidade sob a priori uniforme apresentam melhores resultados que os outros intervalos apresentados.

- Em geral o fator de Bayes sob a priori uniforme consegue detectar melhor a assimetria dos dados.

Concluimos que, em temos gerais a inferência bayesiana sob a priori uniforme tem um melhor desempenho que a baseada na priori de Jeffreys ou a inferência clássica. 


\section{Capítulo 5}

\section{Regressão Linear com erros assimétricos}

\subsection{Introdução}

Neste capítulo apresentaremos o modelo de regressão linear simples com erros normais assimétricos. Em seu desenvolvimento, na Seção 5.2 apresentaremos a função escore, matriz de informação de Fisher e a estimação dos parâmetros de regressão via algoritmo EM. Na Seção 5.3 trataremos este modelo sob o enfoque bayesiano, apresentando a sua forma hierárquica, a distribuição a posteriori aumentada e as distribuições condicionais completas, as quais, podem ser utilizadas para implementar o amostrador de Gibbs e obter a inferência a posteriori.

Além disso, apresentaremos uma aplicação a um conjunto de dados bastante conhecido na literatura, com informações provinientes de um grupo de atletas australianos. Todos os cálculos serão baseados numa amostra de Monte Carlo obtida via amostrador de Gibbs, o qual foi implementada no pacote $R$ e cujo programa 
encontra-se no apêndice D.

\subsection{Inferência Clássica}

O modelo de regressão linear simples é representado por

$$
y_{i}=x_{i}^{T} \beta+\epsilon_{i}
$$

em que $y_{i}$ é a variável reposta para observação $i, \operatorname{com} i=1,2, \ldots, n, x_{i}^{T}=\left(1, x_{i 1}, \ldots, x_{i k}\right)$ representa os valores de $k+1(k+1<n)$ variáveis explicativas que são assumidas fixas e conhecidas, $\beta=\left(\beta_{0}, \beta_{1}, \ldots \beta_{k}\right)^{T}$ é um vetor de parâmetros desconhecidos a serem estimados $\left(\beta \in \Re^{k+1}\right)$. Neste trabalho, vamos substituir a usual suposição de normalidade para os erros, pela seguinte suposição mais geral

$$
\epsilon_{i} \sim S N\left(0, \sigma^{2}, \lambda\right)
$$

Observe que ao fazermos essa suposição não estamos considerando média zero para os erros, pois, $E\left(\epsilon_{i}\right) \neq 0$. Portanto, $E\left(y_{i}\right) \neq x_{i}^{T} \beta$, para obtermos a reta de regressão como a esperança da variável resposta devemos fazer a seguinte correção ao intercepto,

$$
\beta_{0}^{*}=\beta_{0}+\sqrt{\frac{2}{\pi}} \frac{\lambda}{\sqrt{1+\lambda^{2}}} \sigma
$$

assim obtemos

$$
E\left(y_{i}\right)=x_{i}^{T} \beta^{*} \text { sendo } \beta^{*}=\left(\beta_{0}^{*}, \beta_{1}, \ldots \beta_{k}\right)^{T}
$$

Agrupando os $y_{i}$ no vetor $y$ de dimensão $(n \times 1)$, e os $x_{i}^{T}$ na matriz $X$ de dimensão $(n \times p)$, assim temos que a função de verossimilhança baseada numa amostra de $n$ observações independentes pode ser representada por

$$
L(\beta, \sigma, \lambda \mid X, y)=\frac{1}{\sigma^{n}} \exp \left(-\frac{1}{2 \sigma^{2}}(y-X \beta)^{T}(y-X \beta)\right) \prod_{i=1}^{n} \Phi\left(\lambda \frac{y_{i}-x_{i}^{T} \beta}{\sigma}\right),
$$


e o logaritmo natural da função de verossimilhança como

$$
l(\beta, \sigma, \lambda \mid X, y)=-n \log \sigma-\frac{1}{2 \sigma^{2}}(y-X \beta)^{T}(y-X \beta)+\sum_{i=1}^{n} \log \Phi\left(\lambda \frac{y_{i}-x_{i}^{T} \beta}{\sigma}\right)
$$

A função score é obtida derivando-se o logaritmo da função de verossimilhança com respeito a cada um dos parâmetros desconhecidos. De (5.4) segue que

$$
\begin{aligned}
& \frac{\partial l}{\partial \beta}=\frac{1}{\sigma^{2}} X^{T}(y-X \beta)-\frac{\lambda}{\sigma} X^{T} \Lambda_{1} . \\
& \frac{\partial l}{\partial \sigma}=-\frac{n}{\sigma}+\frac{1}{\sigma^{3}}(y-X \beta)^{T}(y-X \beta)-\frac{\lambda}{\sigma} \sum_{i=1}^{n}\left(\frac{y_{i}-x_{i}^{T} \beta}{\sigma}\right) \frac{\phi\left(\lambda \frac{y_{i}-x_{i}^{T} \beta}{\sigma}\right)}{\Phi\left(\lambda \frac{y_{i}-x_{i}^{T} \beta}{\sigma}\right)} \\
& \frac{\partial l}{\partial \lambda}=\sum_{i=1}^{n}\left(\frac{y_{i}-x_{i}^{T} \beta}{\sigma}\right) \frac{\phi\left(\lambda \frac{y_{i}-x_{i}^{T} \beta}{\sigma}\right)}{\Phi\left(\lambda \frac{y_{i}-x_{i}^{T} \beta}{\sigma}\right)}
\end{aligned}
$$

sendo

$$
\Lambda_{1}=\left(\frac{\phi\left(\lambda \frac{y_{1}-x_{1}^{T} \beta}{\sigma}\right)}{\Phi\left(\lambda \frac{y_{1}-x_{1}^{T} \beta}{\sigma}\right)}, \ldots, \frac{\phi\left(\lambda \frac{y_{n}-x_{n}^{T} \beta}{\sigma}\right)}{\Phi\left(\lambda \frac{y_{n}-x_{n}^{T} \beta}{\sigma}\right)}\right)
$$

O nosso próximo passo é obter a matriz de informação de Fisher para $(\beta, \sigma, \lambda)$. De (5.5a) a derivada de $2^{a}$ ordem em relação a $\beta$ e $\beta^{T}$ é

$$
\frac{\partial^{2} l}{\partial \beta \partial \beta^{T}}=-\frac{1}{\sigma^{2}} X^{T} X-\frac{\lambda^{2}}{\sigma^{2}} X^{T} \Lambda_{2} X
$$

sendo

$$
\Lambda_{2}=\operatorname{diag}\left\{\lambda \frac{y_{i}-x_{i}^{T} \beta}{\sigma} \frac{\phi\left(\lambda \frac{y_{i}-x_{i}^{T} \beta}{\sigma}\right)}{\Phi\left(\lambda \frac{y_{i}-x_{i}^{T} \beta}{\sigma}\right)}+\frac{\phi^{2}\left(\lambda \frac{y_{i}-x_{i}^{T} \beta}{\sigma}\right)}{\Phi^{2}\left(\lambda \frac{y_{i}-x_{i}^{T} \beta}{\sigma}\right)}\right\}_{i=1, \ldots, n}
$$


Diferenciando a expressão em (5.5b) com respeito a $\sigma$, temos que

$$
\begin{aligned}
\frac{\partial^{2} l}{\partial \sigma^{2}} & =\frac{n}{\sigma^{2}}-\frac{3}{\sigma} \sum_{i=1}^{n}\left(\frac{y_{i}-x_{i}^{T} \beta}{\sigma}\right)^{2}+\frac{1}{\sigma^{2}} \sum_{i=1}^{n}-2 \lambda\left(\frac{y_{i}-x_{i}^{T} \beta}{\sigma}\right) \frac{\phi\left(\lambda \frac{y_{i}-x_{i}^{T} \beta}{\sigma}\right)}{\Phi\left(\lambda \frac{y_{i}-x_{i}^{T} \beta}{\sigma}\right)} \\
& +\frac{\lambda^{3}}{\sigma}\left(\frac{y_{i}-x_{i}^{T} \beta}{\sigma}\right)^{3} \frac{\phi\left(\lambda \frac{y_{i}-x_{i}^{T} \beta}{\sigma}\right)}{\Phi\left(\lambda \frac{y_{i}-x_{i}^{T} \beta}{\sigma}\right)}+\frac{\lambda^{2}}{\sigma^{2}}\left(\frac{y_{i}-x_{i}^{T} \beta}{\sigma}\right)^{2} \frac{\phi^{2}\left(\lambda \frac{y_{i}-x_{i}^{T} \beta}{\sigma}\right)}{\Phi^{2}\left(\lambda \frac{y_{i}-x_{i}^{T} \beta}{\sigma}\right)} .
\end{aligned}
$$

Finalmente, de (5.5c) a $2^{a}$ derivada com respeito a $\lambda$ pode ser escrita como

$$
\frac{\partial^{2} l}{\partial \lambda^{2}}=-\sum_{i=1}^{n} \lambda\left(\frac{y_{i}-x_{i}^{T} \beta}{\sigma}\right)^{3} \frac{\phi\left(\lambda \frac{y_{i}-x_{i}^{T} \beta}{\sigma}\right)}{\Phi\left(\lambda \frac{y_{i}-x_{i}^{T} \beta}{\sigma}\right)}+\left(\frac{y_{i}-x_{i}^{T} \beta}{\sigma}\right)^{2} \frac{\phi^{2}\left(\lambda \frac{y_{i}-x_{i}^{T} \beta}{\sigma}\right)}{\Phi^{2}\left(\lambda \frac{y_{i}-x_{i}^{T} \beta}{\sigma}\right)} .
$$

De (5.5a), a derivada de $2^{a}$ ordem de $l(\beta, \sigma, \lambda)$ com respeito a $\beta$ e $\sigma$ é dada por

$$
\frac{\partial^{2} l}{\partial \beta \partial \sigma}=-\frac{2}{\sigma^{3}} X^{T}(y-X \beta)-\frac{\lambda}{\sigma^{2}} X^{T} \Lambda_{3}
$$

Diferenciando (5.5a) em relação a $\lambda$, obtemos

$$
\frac{\partial^{2} l}{\partial \beta \partial \lambda}=-\frac{1}{\sigma} X^{T} \Lambda_{3}
$$

com

$$
\Lambda_{3}=\left\{\lambda^{2}\left(\frac{y_{i}-x_{i}^{T} \beta}{\sigma}\right)^{2} \frac{\phi\left(\lambda \frac{y_{i}-x_{i}^{T} \beta}{\sigma}\right)}{\Phi\left(\lambda \frac{y_{i}-x_{i}^{T} \beta}{\sigma}\right)}+\lambda \frac{y_{i}-x_{i}^{T} \beta}{\sigma} \frac{\phi^{2}\left(\lambda \frac{y_{i}-x_{i}^{T} \beta}{\sigma}\right)}{\Phi^{2}\left(\lambda \frac{y_{i}-x_{i}^{T} \beta}{\sigma}\right)}+\frac{\phi\left(\lambda \frac{y_{i}-x_{i}^{T} \beta}{\sigma}\right)}{\Phi\left(\lambda \frac{y_{i}-x_{i}^{T} \beta}{\sigma}\right)}\right\}_{i=1, \ldots, n}
$$

De (5.5b) a derivadada de $2^{a}$ ordem com respeito a $\sigma$ e $\lambda$ é dada por

$$
\begin{aligned}
\frac{\partial^{2} l}{\partial \sigma \partial \lambda} & =-\sum_{i=1}^{n} \frac{1}{\sigma}\left(\frac{y_{i}-x_{i}^{T} \beta}{\sigma}\right) \frac{\phi\left(\lambda \frac{y_{i}-x_{i}^{T} \beta}{\sigma}\right)}{\Phi\left(\lambda \frac{y_{i}-x_{i}^{T} \beta}{\sigma}\right)}+\lambda^{2}\left(\frac{y_{i}-x_{i}^{T} \beta}{\sigma}\right)^{3} \frac{\phi\left(\lambda \frac{y_{i}-x_{i}^{T} \beta}{\sigma}\right)}{\Phi\left(\lambda \frac{y_{i}-x_{i}^{T} \beta}{\sigma}\right)} \\
& +\frac{\lambda}{\sigma}\left(\frac{y_{i}-x_{i}^{T} \beta}{\sigma}\right)^{2} \frac{\phi^{2}\left(\lambda \frac{y_{i}-x_{i}^{T} \beta}{\sigma}\right)}{\Phi^{2}\left(\lambda \frac{y_{i}-x_{i}^{T} \beta}{\sigma}\right)} .
\end{aligned}
$$


Finalmente, a matriz de informação de Fisher para os parâmetros $\left(\beta, \sigma^{2}, \lambda\right)$ pode ser escrita como

$\left[\begin{array}{ccc}\frac{1+\lambda^{2} a_{0}}{\sigma^{2}} X^{T} X & \frac{\sqrt{2 / \pi} \lambda\left(1+2 \lambda^{2}\right)\left(1+\lambda^{2}\right)^{-\frac{3}{2}}+\lambda^{2} a_{1}}{\sigma^{2}} X^{T} 1 & \frac{\sqrt{2 / \pi}\left(1+\lambda^{2}\right)^{-\frac{3}{2}}-\lambda a_{1}}{\sigma^{2}} X^{T} 1 \\ \frac{\sqrt{2 / \pi} \lambda\left(1+2 \lambda^{2}\right)\left(1+\lambda^{2}\right)^{-\frac{3}{2}}+\lambda^{2} a_{1}}{\sigma^{2}} 1^{T} X & n \frac{2+\lambda^{2} a_{2}}{\sigma^{2}} & -n \frac{\lambda a_{2}}{\sigma} \\ \frac{\sqrt{2 / \pi}\left(1+\lambda^{2}\right)^{-\frac{3}{2}}-\lambda a_{1}}{\sigma^{2}} 1^{T} X & -n \frac{\lambda a_{2}}{\sigma} & n a_{2}\end{array}\right]$

sendo 1 um vetor coluna de uns de tamanho $n$.

Notamos que não existem soluções explicitas para o problema de maximização da função de verossimilhança dada em (5.3). Neste caso podemos maximizar numericamente usando softwares existentes como, por exemplo, o Matlab, R e S-plus. Estes softwares contém rotinas prontas para tratar problemas de maximização (minimização) de qualquer função.

Entretanto, similarmente ao que foi feito no capítulo 3, propomos aqui um algoritmo EM para o cálculo dos e.m.v. nos valendo da representação de Henze (1986) e introduzindo uma reparametrização de modo a obtermos formas fechadas para o algoritmo EM no passo M.

Para obter soluções fechadas do máximo da esperança do logaritmo da função de verossimilhança (Passo $\mathrm{M}$ ), utilizamos a mesma reparametrização considerada anteriormente $\eta=\sigma \delta$ e $\tau=\sigma \sqrt{1-\delta^{2}}$ e a forma hierárquica do modelo

$$
\begin{aligned}
y_{i} \mid U_{i} & \sim N\left(x_{i}^{T} \beta+\eta U_{i}, \tau^{2}\right) \\
U_{i} & \sim H N(0,1) .
\end{aligned}
$$

O logaritmo natural da função de verossimilhança aumentada baseada numa 
amostra de $n$ observações independentes, na nova reparametrização é dada por

$$
l_{c}(\theta)=-n \log \tau-\frac{1}{2 \tau^{2}}\left[(y-X \beta-\eta U)^{T}(y-X \beta-\eta U)\right]-\frac{1}{2} U^{T} U
$$

Após algumas manipulações algébricas obtemos,

$$
U_{i} \mid D, \theta \sim N\left(\left(y_{i}-x_{i}^{T} \beta\right) \frac{\eta}{\tau^{2}+\eta^{2}}, \frac{\tau^{2}}{\tau^{2}+\eta^{2}}\right) I_{[0, \infty)}
$$

com

$$
\begin{aligned}
E\left(U_{i} \mid D, \theta\right) & =\left(y_{i}-\beta x_{i}\right) \frac{\eta}{\tau^{2}+\eta^{2}}+\frac{\tau}{\sqrt{\tau^{2}+\eta^{2}}} \frac{\phi\left(\frac{\eta}{\tau} \frac{\left(y_{i}-\beta x_{i}\right)}{\sqrt{\tau^{2}+\eta^{2}}}\right)}{\Phi\left(\frac{\eta}{\tau} \frac{\left(y_{i}-\beta x_{i}\right)}{\sqrt{\tau^{2}+\eta^{2}}}\right)} \mathrm{e} \\
E\left(U_{i}^{2} \mid D, \theta\right) & =\left(y_{i}-\beta x_{i}\right)^{2} \frac{\eta^{2}}{\left(\tau^{2}+\eta^{2}\right)^{2}}+\frac{\tau^{2}}{\tau^{2}+\eta^{2}} \\
& +\left(y_{i}-\beta x_{i}\right) \frac{\eta}{\tau^{2}+\eta^{2}} \frac{\tau}{\sqrt{\tau^{2}+\eta^{2}}} \frac{\phi\left(\frac{\eta}{\tau} \frac{\left(y_{i}-\beta x_{i}\right)}{\sqrt{\tau^{2}+\eta^{2}}}\right)}{\Phi\left(\frac{\eta}{\tau} \frac{\left(y_{i}-\beta x_{i}\right)}{\sqrt{\tau^{2}+\eta^{2}}}\right)} .
\end{aligned}
$$

Consideramos a seguinte notação para $\widehat{U_{1}}$ e $\widehat{U_{2}}$

$$
\begin{aligned}
& \widehat{U_{1}}=\left(E\left(U_{1} \mid D, \widehat{\theta}\right), E\left(U_{2} \mid D, \widehat{\theta}\right), \ldots, E\left(U_{n} \mid D, \widehat{\theta}\right)\right) \\
& \widehat{U_{2}}=\left(E\left(U_{1}^{2} \mid D, \widehat{\theta}\right), E\left(U_{2}^{2} \mid D, \widehat{\theta}\right), \ldots, E\left(U_{n}^{2} \mid D, \widehat{\theta}\right)\right)
\end{aligned}
$$

Temos que a esperança do logaritmo da função de verossimilhança aumentada denotada por $Q(\theta, \widehat{\theta})$ é dada por

$$
Q(\theta, \widehat{\theta})=E\left(l_{c}(\theta \mid y, X, \widehat{\theta})\right)-n \log \tau-\frac{1}{2 \tau^{2}}\left[(Y-X \beta)^{T}(Y-X \beta)-2 \eta(Y-X \beta)^{T} \widehat{U_{1}}+\eta^{2} \widehat{U_{2}}\right] .
$$


A seguir calculamos a primeira derivada de $Q(\theta, \widehat{\theta})$,

$$
\begin{aligned}
& \frac{\partial Q}{\partial \beta}=-\frac{1}{2 \tau^{2}}\left(-2 X^{T} y+2 X^{T} X \beta+2 \eta X^{T} \widehat{U}_{1}\right) \\
& \frac{\partial Q}{\partial \eta}=-\frac{1}{2 \tau^{2}}\left(-2(y-X \beta)^{T} \widehat{U}_{1}+2 \eta 1^{T} \widehat{U}_{2}\right) \\
& \frac{\partial Q}{\partial \tau}=-\frac{n}{\tau}+\frac{1}{\tau^{3}}\left[(y-X \beta)^{T}(y-X \beta)-2 \eta(y-X \beta)^{T} \widehat{U}_{1}+\eta^{2} \widehat{U}_{2}\right] .
\end{aligned}
$$

Logo, o ponto que maximiza $Q(\theta, \widehat{\theta})$ é $(\widehat{\beta}, \widehat{\tau}, \widehat{\eta})$, cujas expressões estão dadas a seguir

$$
\begin{aligned}
& \widehat{\eta}=\frac{y^{T}\left(I-X\left(X^{T} X\right)^{-1} X^{T}\right) \widehat{U}_{1}}{1^{T} \widehat{U}_{2}-\widehat{U}_{1}^{T} X\left(X^{T} X\right)^{-1} X^{T} \widehat{U}_{1}} \\
& \widehat{\beta}=\left(X^{T} X\right)^{-1} X^{T}\left(y-\widehat{\eta} \widehat{U}_{1}\right) \\
& \widehat{\tau}=\left\{\frac{1}{n}\left[(y-X \widehat{\beta})^{T}(y-X \widehat{\beta})-2 \widehat{\eta}(y-X \widehat{\beta})^{T} \widehat{U}_{1}+\widehat{\eta}^{2} 1^{T} \widehat{U_{2}}\right]\right\}^{\frac{1}{2}}
\end{aligned}
$$

A seguir apresentamos os passos para a implementação do algoritmo EM

\section{Passo E}

Dado $\theta=\widehat{\theta}$, calcular $\widehat{U_{1}}$ e $\widehat{U_{1}}$, com as expressões dadas em (5.9),

\section{Passo M}

Atualizar $\widehat{\theta}$, maximizando (5.10) sob $\theta$, utilizando (5.11). 


\subsection{Inferência Bayesiana}

Como foi discutido na seção anterior o modelo de regressão definido em (5.1) e (5.2) pode ser escrito na forma hirárquica, apresentada em (5.6)

Neste caso, a função de verossimilhança aumentada é expressa por

$$
L(\beta, \tau, \eta \mid y, X, U)=-\frac{1}{\tau^{n}} \exp \left[-\frac{1}{2 \tau^{2}}(y-X \beta-\eta U)^{T}(y-X \beta-\eta U)\right] \exp \left(-\frac{1}{2} U^{T} U\right)
$$

A distribuição a priori a ser utilizada neste modelo é similar a proposta para o modelo de três parâmetros, isto é,

$$
p(\lambda, \beta, \sigma) \propto \frac{1}{\sigma} p(\lambda)
$$

onde $\lambda \sim t\left(0, \sigma_{t}^{2}, k\right)$. Lembremos que, quando $k=\frac{1}{2}$ e $\sigma_{t}^{2}=\frac{\pi^{2}}{4}$, obtemos a aproximação da priori de Jeffreys e quando $k=2$ e $\sigma_{t}^{2}=\frac{1}{2}$, a priori induzida pela uniforme $\delta \sim U(-1,1)$. Considerando a representação hierárquica da distribuição $t$-Student e mudando a parametrização como foi determinado em (3.28), obtemos

$$
f(\beta, \tau, \eta, w) \propto \frac{1}{\tau^{2}} \exp \left(-\frac{1}{2} \frac{\eta^{2} w}{\tau^{2} \sigma_{t}^{2}}\right) w^{\frac{k+1}{2}-1} \exp \left(-\frac{k}{2} w\right) .
$$

Assim, temos que a função de distribuição a posteriori aumentada é expressa por

$$
f(\beta, \tau, \eta, w, U \mid y, X) \propto f(\beta, \tau, \eta, w) L(\beta, \tau, \eta \mid y, X, U) .
$$

Finalmente, de (5.15) obtivemos as distribuições condicionais completas dadas pelas seguintes expressões 


$$
\begin{aligned}
& w \mid \beta, \tau, \eta, U, y \sim \operatorname{Gamma}\left(\frac{k+1}{2}, \frac{1}{2}\left(\frac{\eta^{2}}{\sigma_{t}^{2} \tau^{2}}+k\right)\right) \\
& u_{i} \mid \beta, \tau, \eta, w, y \sim N\left(\frac{\left(y_{i}-x_{i}^{T} \beta\right) \eta}{\tau^{2}+\eta^{2}}, \frac{\tau^{2}}{\tau^{2}+\eta^{2}}\right) I_{[0, \infty)}\left(u_{i}\right) \forall i, i=1, \ldots, n \\
& \beta \mid \tau, \eta, w, U, y \sim N_{k+1}\left(\left(X^{T} X\right)^{-1} X^{T}(y-\eta U), \tau^{2}\left(X^{T} X\right)^{-1}\right) \\
& \eta \mid \beta, \tau, w, U, y \sim N\left(\frac{(y-X B)^{T} U}{\frac{w}{\sigma_{t}^{2}}+\sum_{i=1}^{n} u_{i}^{2}}, \frac{\frac{w}{\sigma_{t}^{2}}+\sum_{i=1}^{n} u_{i}^{2}}{2}\right) \\
& \frac{1}{\tau^{2}} \mid \beta, \eta, w, U, y \sim \operatorname{Gamma}\left(\frac{n+1}{2}, \frac{1}{2}\left((Y-\beta X-\eta U)^{T}(Y-\beta X-\eta U)+\frac{w \eta^{2}}{\sigma_{t}^{2}}\right)\right)
\end{aligned}
$$

\subsection{Aplicação}

Considerando os dados dos atletas australianos, incluido no pacote $s n$ do programa $R$, disponível em http : //azzalini.stat.unipd.it/SN/. Definimos o seguinte modelo

$$
B f a t_{i}=\beta_{0}+\beta_{1} W t_{i}+\epsilon_{i}, \quad i=1, \ldots, 202
$$

sendo $B f a t_{i}$, a porcentagem de gordura corporal de o i-ésimo sujeito e $W t_{i}$ o peso do o i-ésimo sujeito. Assumimos também que os $\epsilon_{i} \sim S N\left(0, \sigma^{2}, \lambda\right)$. Calculamos o e.m.v., o máximo, a média e a mediana a posteriori para cada um dos parâmetros do modelo. Os resultados são apresentados na tabela a seguir 
Tabela 5.1: Estimativas pontuais dos parâmetros para os dados AIS

\begin{tabular}{|c|c|c|c|c|c|c|c|}
\hline & & \multicolumn{3}{|c|}{ Priori I : Jeffreys } & \multicolumn{3}{c|}{ Priori II : Uniforme } \\
\hline Param & e.m.v. & Máximo & Média & Mediana & Máximo & Média & Mediana \\
\hline$\lambda$ & $\infty$ & 51.043 & $\infty$ & 87.34 & 35.753 & 52.500 & 40.260 \\
$\beta_{0}$ & 3.589 & 3.488 & 4.340 & 4.204 & 4.318 & 4.251 & 4.187 \\
$\beta_{1}$ & 0.029 & 0.020 & 0.018 & 0.021 & 0.020 & 0.020 & 0.021 \\
$\sigma$ & 9.953 & 9.783 & 9.964 & 9.978 & 9.754 & 10.000 & 9.972 \\
$\beta_{0}^{*}$ & 11.533 & 12.051 & 12.254 & 12.214 & 11.953 & 12.144 & 12.131 \\
\hline
\end{tabular}

Verifica-se que as estimativas de $\beta$ e $\sigma$ são pouco sensíveis em relação a escolha da priori e diferem pouco das estimativas de máxima verossimilhança. No entanto, para $\lambda$ observamos que o e.m.v. e a média posteriori sob a priori de Jeffreys são infinitos. Os valores do máximo a posteriori sob a priori de Jeffreys (52.51) e a média a posteriori sob a priori uniforme (52.50) são bastante similares. Baseados nas conclusões do estudo de simulação desenvolvido no capítulo 4, consideramos estas estimativas as mais confiáveis. Logo, os dados são bastante assimétricos.

Para testar a hipótese de nomalidade, $H_{0}: \lambda=0$ vs $H_{1}: \lambda \neq 0$, utilizamos o teste de razão de verossimilhanças, o qual fornece um valor da estatística de 163.071 com um nível descritivo de 0.0000 . Portanto, rejeita-se a hipótese nula. 


\section{Capítulo 6}

\section{Considerações Finais}

Neste trabalho consideramos uma nova reparametrização do modelo normal assimétrico que, na aborbagem clássica, nos permitiu obter formas fechadas no passo M do algoritmo EM e, na abordagem bayesiana, formas conhecidas para as distribuições condicionais a posteriori. Apresentamos também, boas aproximações para a priori de Jeffreys e a matriz de informação de Fisher.

Os resultados do estudo de simulação mostraram que na maioria dos casos a média a posteriori sob a priori uniforme e o máximo a posteriori sob a priori de Jeffreys tem um melhor desempenho que os outros estimadores, em relação ao viés e o erro quadrático médio. Em relação a estimação intervalar, os intervalos de credibilidades sob a priori uniforme mostraram-se superiores aos outros intervalos. No contexto de teste de hipóteses, o fator de Bayes sob a priori uniforme conseguiu detectar melhor a assimetria dos dados do que o teste de razão de verossimilhanças e o fator de Bayes sob a priori de Jeffreys. Assim, concluimos que o uso da priori uniforme é uma boa alternativa para se fazer inferência sob o parâmetro de assimetria do modelo.

Este estudo da distribuição normal assimétrica nos oferece várias possibilidades 
para futuras pesquisas, entre estas podemos destacar as seguintes:

- Melhorar a convergência do amostrador de Gibbs utilizando a metodologia descrita em Liu \& Wu (1999), Liu (2003a) e Liu (2003b).

- Implementar o algoritmo PX-EM Liu, Rubin \& Wu (1998) e Liu (2003b), para acelerar a convergência do algoritmo EM.

- Extender o estudo da distribuição normal assimétrica para uma classe mais ampla de distribuições assimétricas, como por exemplo, a distribuição $t$-Student assimétrica. Considerando a abordagem bayesiana, temos como desafio a obtenção de distribuições a priori de Jeffreys e de referência (Berger \& Bernardo, 1992).

- Estudar o modelo de regressão com erros nas variáveis utilizando a nova reparametrização descrita neste trabalho. Acreditamos ser fácil neste caso, obter formas fechadas no passo $\mathrm{M}$ do algoritmo EM e, na implementação do algorimo de Gibbs, formas conhecidas para as distribuições condicionais completas. 


\section{Apêndice A}

\section{Aproximações}

Proposição A.1 A matriz de informação de Fisher $I(\lambda)$ da distribuição normal assimétrica padrão pode ser aproximada pela seguinte expressão

$$
I(\lambda) \approx \frac{2}{\pi}\left(1+\frac{2 \lambda^{2}}{\left(\pi^{2} / 4\right)}\right)^{-\frac{3}{2}}
$$

Prova:

Para obter o resultado dado na proposição A.1, utilizamos a seguinte aproximação de funções obtida por Chaibub Neto \& Branco (2003),

$$
\frac{1}{\pi} \frac{\phi(z)}{\sqrt{\Phi(z)[1-\Phi(z)]}} \approx \frac{1}{\sqrt{2 \pi}(\pi / 2)} \exp \left(-\frac{z^{2}}{2\left(\pi^{2} / 4\right)}\right) .
$$


Observamos que

$$
\begin{aligned}
I(\lambda) & =\int_{-\infty}^{\infty} 2 z^{2} \phi(z) \frac{\phi^{2}(\lambda z)}{\Phi(\lambda z)} d z \\
& =\int_{0}^{\infty} 2 z^{2} \phi(z) \frac{\phi^{2}(\lambda z)}{\Phi(\lambda z)} d z+\int_{-\infty}^{0} 2 z^{2} \phi(z) \frac{\phi^{2}(\lambda z)}{\Phi(\lambda z)} d z \\
& =\int_{0}^{\infty} 2 z^{2} \phi(z) \frac{\phi^{2}(\lambda z)}{\Phi(\lambda z)} d z+\int_{0}^{\infty} 2 z^{2} \phi(z) \frac{\phi^{2}(\lambda z)}{1-\Phi(\lambda z)} d z \\
& =\int_{0}^{\infty} 2 z^{2} \phi(z) \frac{\phi^{2}(\lambda z)}{\Phi(\lambda z)[1-\Phi(\lambda z)]} d z .
\end{aligned}
$$

Logo, substituindo (A.2) na expresão acima e considerando a seguinte notação $\phi(. \mid$ $a, b)$ para representar uma distribuição normal com média $a$ e variância $b$, temos que

$$
\begin{aligned}
I(\lambda) & \approx \int_{0}^{\infty} 2 z^{2} \phi(z) \frac{\pi}{2} \frac{1}{\left(\pi^{2} / 4\right)} \exp \left(-\frac{\lambda^{2} z^{2}}{\left(\pi^{2} / 4\right)}\right) d z \\
& =\frac{\pi}{\left(\pi^{2} / 4\right)}\left(1+\frac{2 \lambda^{2}}{\left(\pi^{2} / 4\right)}\right)^{-1 / 2} \int_{0}^{\infty} z^{2} \phi\left(z \mid 0,\left(1+\frac{2 \lambda^{2}}{\left(\pi^{2} / 4\right)}\right)^{-1}\right) d z \\
& =\frac{2}{\pi}\left(1+\frac{2 \lambda^{2}}{\left(\pi^{2} / 4\right)}\right)^{-\frac{3}{2}} .
\end{aligned}
$$

Corolário A.1 A priori de Jeffreys $f^{J}(\lambda)$ associada ao modelo normal assimétrico padrão é aproximada por

$$
f^{J}(\lambda) \approx t\left(0, \frac{\pi^{2}}{4}, \frac{1}{2}\right)
$$

Prova : Lembremos que $f^{J}(\lambda)=I^{\frac{1}{2}}(\lambda)$ então

$$
f^{J}(\lambda) \approx \sqrt{\frac{2}{\pi}}\left(1+\frac{2 \lambda^{2}}{\left(\pi^{2} / 4\right)}\right)^{-\frac{3}{4}} .
$$

que é o kernel de uma distribuição t-student especificada em (A.3). 
Proposição A.2 Seja $a_{k}=E\left[z^{k}\left(\frac{\phi(\lambda z)}{\Phi(\lambda z)}\right)^{2}\right], k=0,1,2, \ldots$, onde os valores esperados são avaliados na distribuição normal assimétrica padrão. Uma boa aproximação para $a_{k}$ quando $k$ é par, é dada pela seguinte expressão

$$
a_{k} \approx \frac{1}{2^{\frac{k}{2}-1} \pi} \frac{k !}{(k / 2) !}\left(1+\frac{2 \lambda^{2}}{\left(\pi^{2} / 4\right)}\right)^{-\frac{k+1}{2}} .
$$

Prova: Observamos que

$$
\begin{aligned}
a_{k} & =\int_{-\infty}^{\infty} 2 z^{k} \phi(z) \frac{\phi^{2}(\lambda z)}{\Phi(\lambda z)} d z \\
& =\int_{0}^{\infty} 2 z^{k} \phi(z) \frac{\phi^{2}(\lambda z)}{\Phi(\lambda z)} d z+\int_{-\infty}^{0} 2 z^{k} \phi(z) \frac{\phi^{2}(\lambda z)}{\Phi(\lambda z)} d z \\
& =\int_{0}^{\infty} 2 z^{k} \phi(z) \frac{\phi^{2}(\lambda z)}{\Phi(\lambda z)} d z+\int_{0}^{\infty} 2 z^{k} \phi(z) \frac{\phi^{2}(\lambda z)}{1-\Phi(\lambda z)} d z \\
& =\int_{0}^{\infty} 2 z^{k} \phi(z) \frac{\phi^{2}(\lambda z)}{\Phi(\lambda z)[1-\Phi(\lambda z)]} d z .
\end{aligned}
$$

Logo, substituindo (A.2) na expresão acima, temos que

$$
\begin{aligned}
a_{k} & \approx \int_{0}^{\infty} 2 z^{k} \phi(z) \frac{\pi}{2} \frac{1}{\left(\pi^{2} / 4\right)} \exp \left(-\frac{\lambda^{2} z^{2}}{\left(\pi^{2} / 4\right)}\right) d z \\
& =\frac{\pi}{\left(\pi^{2} / 4\right)}\left(1+\frac{2 \lambda^{2}}{\left(\pi^{2} / 4\right)}\right)^{-1 / 2} \int_{0}^{\infty} z^{k} \phi\left(z \mid 0,\left(1+\frac{2 \lambda^{2}}{\left(\pi^{2} / 4\right)}\right)^{-1}\right) d z \\
& =\frac{1}{2^{\frac{k}{2}-1} \pi} \frac{k !}{(k / 2) !}\left(1+\frac{2 \lambda^{2}}{\left(\pi^{2} / 4\right)}\right)^{-\frac{k+1}{2}} .
\end{aligned}
$$


Para o caso em que $k$ é impar, uma aproximação é dada na seguinte proposição

Proposição A.3 Seja $a_{k}=E\left[z^{k}\left(\frac{\phi(\lambda z)}{\Phi(\lambda z)}\right)^{2}\right]$, onde a esperança é avaliada na distribuição normal assimétrica padrão. Uma boa aproximação para $a_{k}$ quando $k$ é impar, é dada por

$$
\begin{aligned}
& a_{k} \approx-\frac{2^{\frac{k+2}{2}}}{\pi^{\frac{3}{2}}}\left(\frac{k-1}{2}\right) ! \lambda\left(1+\frac{2 \lambda^{2}}{\left(\pi^{2} / 4\right)}\right)^{-\frac{k+2}{2}}\left(1+\lambda^{2}\left(1+\frac{2 \lambda^{2}}{\left(\pi^{2} / 4\right)}\right)^{-1}\right)^{-\frac{1}{2}} \\
&\left\{\sum_{j=0}^{\frac{k-1}{2}} c_{j} \lambda^{j}\left(1+\frac{2 \lambda^{2}}{\left(\pi^{2} / 4\right)}\right)^{-\frac{j}{2}}\right\}
\end{aligned}
$$

sendo $c_{0}=1, c_{j}=\frac{2 j-1}{2 j} c_{j-1}$

Prova :

$$
\begin{aligned}
& a_{k}=\int_{-\infty}^{\infty} 2 z^{k} \phi(z) \frac{\phi^{2}(\lambda z)}{\Phi(\lambda z)} d z \\
& =\int_{0}^{\infty} 2 z^{k} \phi(z) \frac{\phi^{2}(\lambda z)}{\Phi(\lambda z)} d z+\int_{-\infty}^{0} 2 z^{k} \phi(z) \frac{\phi^{2}(\lambda z)}{\Phi(\lambda z)} d z \\
& =\int_{0}^{\infty} 2 z^{k} \phi(z) \frac{\phi^{2}(\lambda z)}{\Phi(\lambda z)} d z-\int_{0}^{\infty} 2 z^{k} \phi(z) \frac{\phi^{2}(\lambda z)}{1-\Phi(\lambda z)} d z \\
& =\int_{0}^{\infty} 2 z^{k} \phi(z) \phi^{2}(\lambda z)\left(\frac{1}{\Phi(\lambda z)}-\frac{1}{1-\Phi(\lambda z)}\right) d z \\
& =\int_{0}^{\infty} 2 z^{k} \phi(z) \frac{\phi^{2}(\lambda z)}{\Phi(\lambda z)[1-\Phi(\lambda z)]} d z-\int_{0}^{\infty} 4 z^{k} \phi(z) \frac{\phi^{2}(\lambda z)}{\Phi(\lambda z)[1-\Phi(\lambda z)]} \Phi(\lambda z) d z .
\end{aligned}
$$

Logo, substituindo (A.2) na expresão acima, temos que

$$
\begin{aligned}
a_{k} & \approx \int_{0}^{\infty} 2 z^{k} \phi(z) \frac{\pi}{2} \frac{1}{\left(\pi^{2} / 4\right)} \exp \left(-\frac{\lambda^{2} z^{2}}{\left(\pi^{2} / 4\right)}\right) d z-\int_{0}^{\infty} 4 z^{k} \phi(z) \frac{\pi}{2} \frac{1}{\left(\pi^{2} / 4\right)} \exp \left(-\frac{\lambda^{2} z^{2}}{\left(\pi^{2} / 4\right)}\right) \Phi(\lambda z) d z \\
& =\frac{4}{\pi} \int_{0}^{\infty} z^{k} \phi\left[\left(1+\frac{2 \lambda^{2}}{\left(\pi^{2} / 4\right)}\right)^{1 / 2} z\right] d z-\frac{8}{\pi} \int_{0}^{\infty} z^{k} \phi\left[\left(1+\frac{2 \lambda^{2}}{\left(\pi^{2} / 4\right)}\right)^{1 / 2} z\right] \Phi(\lambda z) d z .
\end{aligned}
$$


Fazendo a mudança de variável $w=\left(1+\frac{2 \lambda^{2}}{\left(\pi^{2} / 4\right)}\right)^{\frac{1}{2}} z$ obtemos

$$
\begin{aligned}
a_{k} & \approx \frac{4}{\pi}\left(\frac{2}{\pi}\right)^{\frac{3}{2}}\left(1+\frac{2 \lambda^{2}}{\left(\pi^{2} / 4\right)}\right)^{-\frac{k+1}{2}} \int_{0}^{\infty} w^{k} \phi(w) d w \\
& -\frac{8}{\pi}\left(\frac{2}{\pi}\right)^{\frac{3}{2}}\left(1+\frac{2 \lambda^{2}}{\left(\pi^{2} / 4\right)}\right)^{-\frac{k+1}{2}} \int_{0}^{\infty} w \phi(w) \Phi\left(\lambda\left(1+\frac{2 \lambda^{2}}{\left(\pi^{2} / 4\right)}\right)^{-\frac{1}{2}} w\right) d w .
\end{aligned}
$$

Finalmente, utilizando os resultados dados em Owen (1980) nas páginas 394 e 399, obtemos

$$
\begin{aligned}
a_{k} & \approx \frac{2^{\frac{k+2}{2}}}{\pi^{\frac{3}{2}}}\left(\frac{k-1}{2}\right) !\left(1+\frac{2 \lambda^{2}}{\left(\pi^{2} / 4\right)}\right)^{-\frac{k+1}{2}}-\frac{2^{\frac{k+2}{2}}}{\pi^{\frac{3}{2}}}\left(\frac{k-1}{2}\right) !\left(1+\frac{2 \lambda^{2}}{\left(\pi^{2} / 4\right)}\right)^{-\frac{k+1}{2}} \\
& \times\left[1+\lambda\left(1+\frac{2 \lambda^{2}}{\left(\pi^{2} / 4\right)}\right)^{-\frac{1}{2}}\left(1+\lambda^{2}\left(1+\frac{2 \lambda^{2}}{\left(\pi^{2} / 4\right)}\right)^{-1}\right)^{-\frac{1}{2}}\left\{\sum_{j=0}^{\frac{k-1}{2}} c_{j} \lambda^{j}\left(1+\frac{2 \lambda^{2}}{\left(\pi^{2} / 4\right)}\right)^{-\frac{j}{2}}\right\}\right] \\
& =-\frac{2^{\frac{k+2}{2}}}{\pi^{\frac{3}{2}}}\left(\frac{k-1}{2}\right) ! \lambda\left(1+\frac{2 \lambda^{2}}{\left(\pi^{2} / 4\right)}\right)^{-\frac{k+2}{2}}\left(1+\lambda^{2}\left(1+\frac{2 \lambda^{2}}{\left(\pi^{2} / 4\right)}\right)^{-1}\right)^{-\frac{1}{2}} \\
& \left\{\sum_{j=0}^{\frac{k-1}{2}} c_{j} \lambda^{j}\left(1+\frac{2 \lambda^{2}}{\left(\pi^{2} / 4\right)}\right)^{-\frac{j}{2}}\right\}
\end{aligned}
$$

Pela proposição A.3 temos que $a_{1}$ é aproximado por

$$
a_{1} \approx-\left(\frac{2}{\pi}\right)^{\frac{3}{2}} \lambda\left(1+\frac{2 \lambda^{2}}{\left(\pi^{2} / 4\right)}\right)^{-\frac{3}{2}}\left(1+\lambda^{2}\left(1+\frac{2 \lambda^{2}}{\left(\pi^{2} / 4\right)}\right)^{-1}\right)^{-\frac{1}{2}}
$$

Utilizando a proposição A.2 podemos calcular uma aproximação para $M(\lambda)$ dado em (3.5)

$$
M(\lambda)=-\frac{\lambda}{2} \frac{a_{4}(\lambda)}{a_{2}(\lambda)} \approx-\frac{3}{2} \lambda\left(1+\frac{2 \lambda^{2}}{\left(\pi^{2} / 4\right)}\right)^{-1} .
$$

As figuras (A.1), (A.2), (A.3), (A.4) e (A.5) ilustram o desempenho das aproximações da matriz de informação de Fisher, a priori de Jeffreys, $a_{0}(\lambda), a_{1}(\lambda)$ e $M(\lambda)$, respectivamente. 
Figura A.1: Matriz de informação de Fisher $I(\lambda)$ e sua aproximação

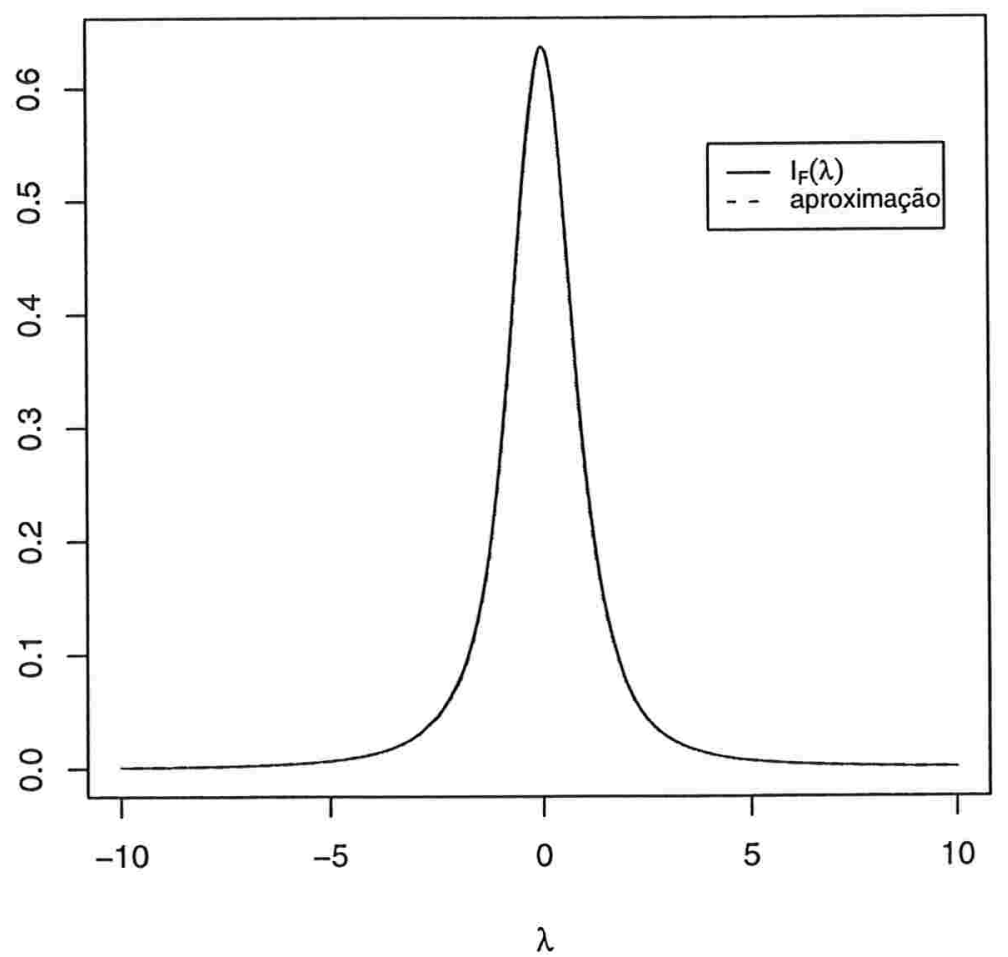


Figura A.2: Priori de Jeffreys $f^{J}(\lambda)$ e sua aproximação

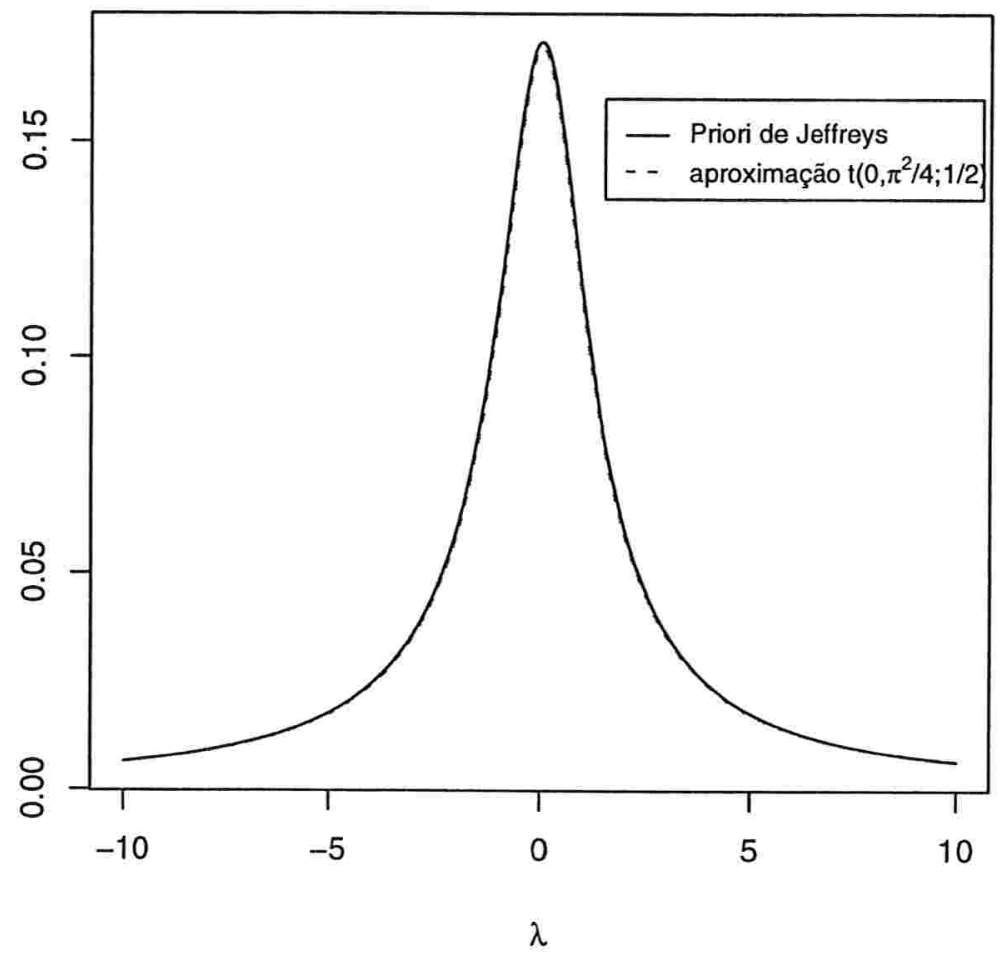


Figura A.3: $a_{0}(\lambda)$ e sua aproximação

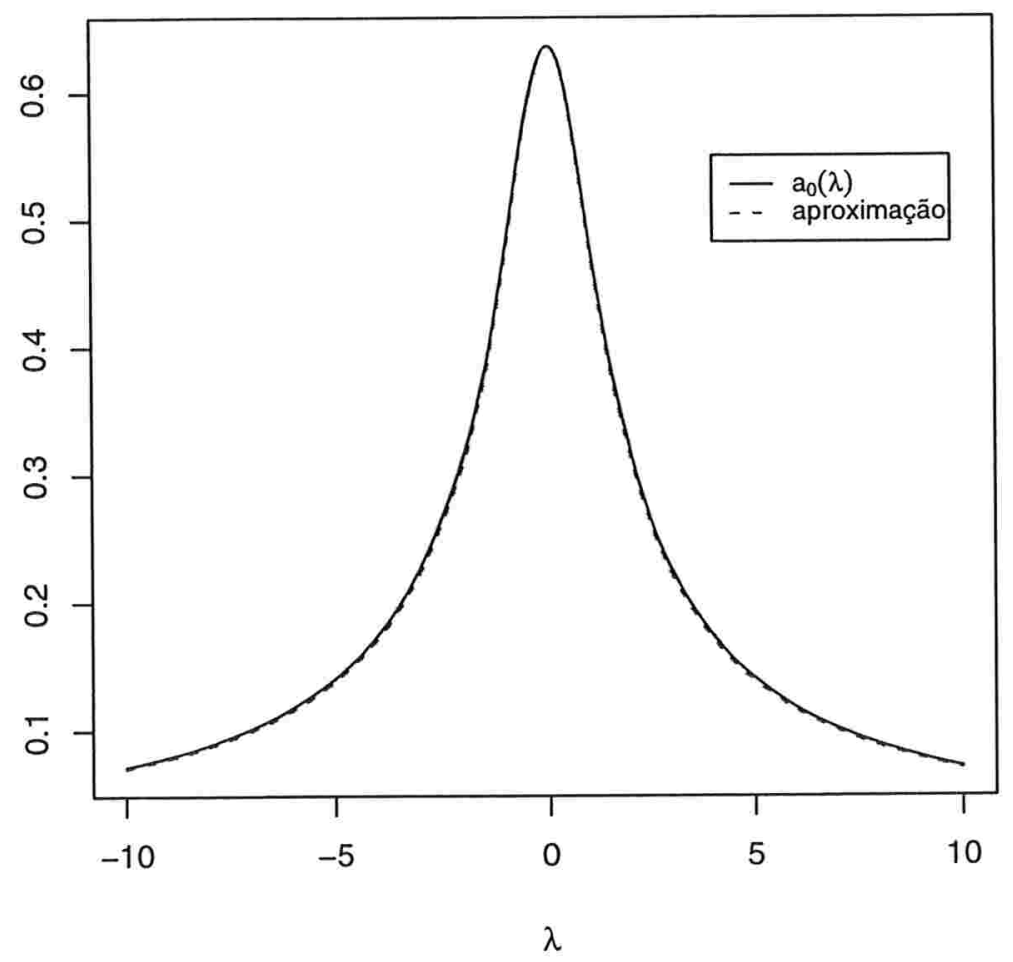


Figura A.4: $a_{1}(\lambda)$ e sua aproximação

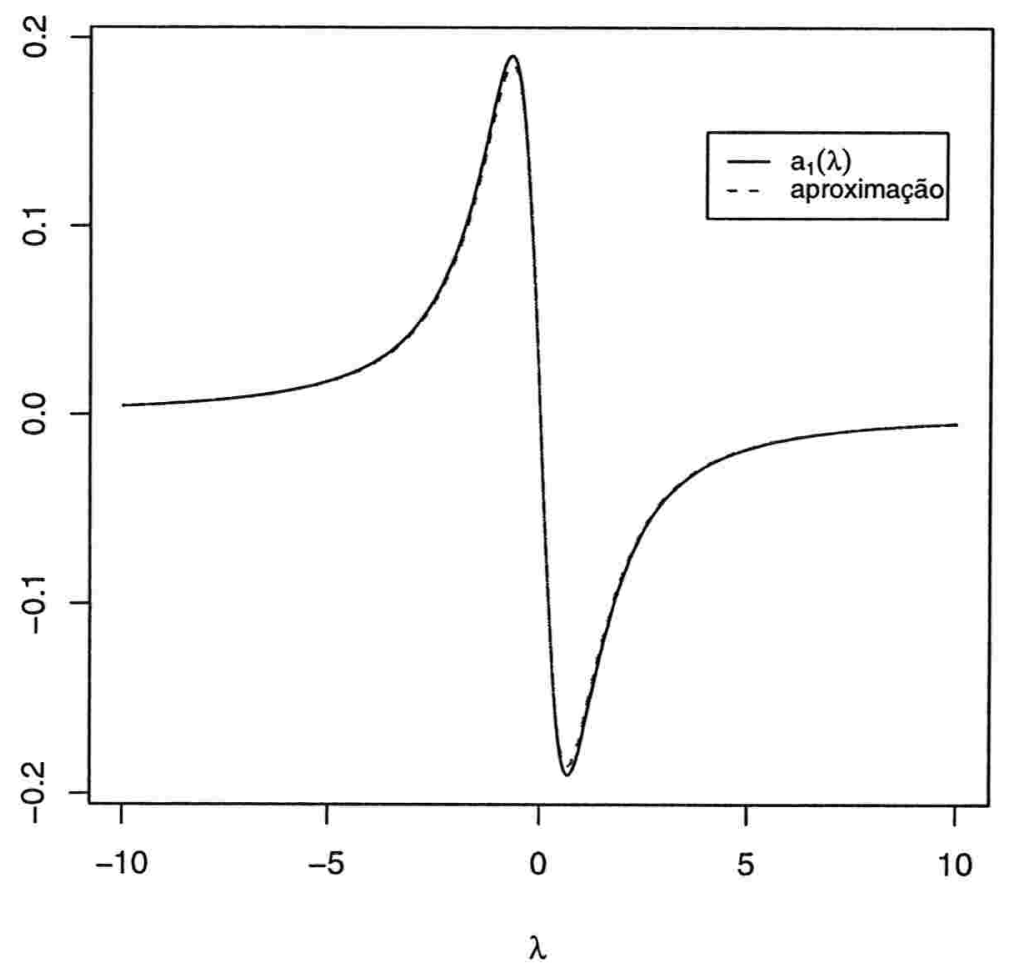


Figura A.5: $M(\lambda)$ e sua aproximação

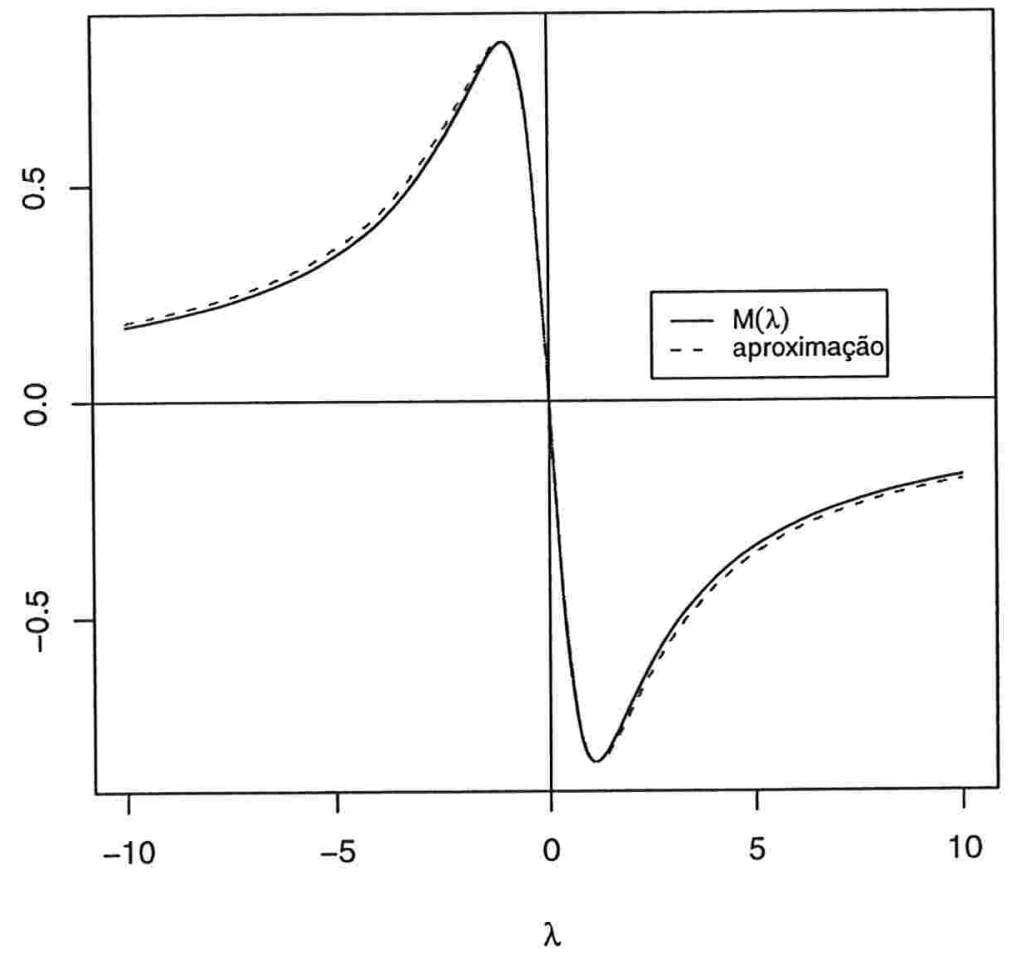




\section{Apêndice B}

\section{Figuras}

Figura B.1: Funções de densidade da normal assimétrica com valores positivos de $\lambda$

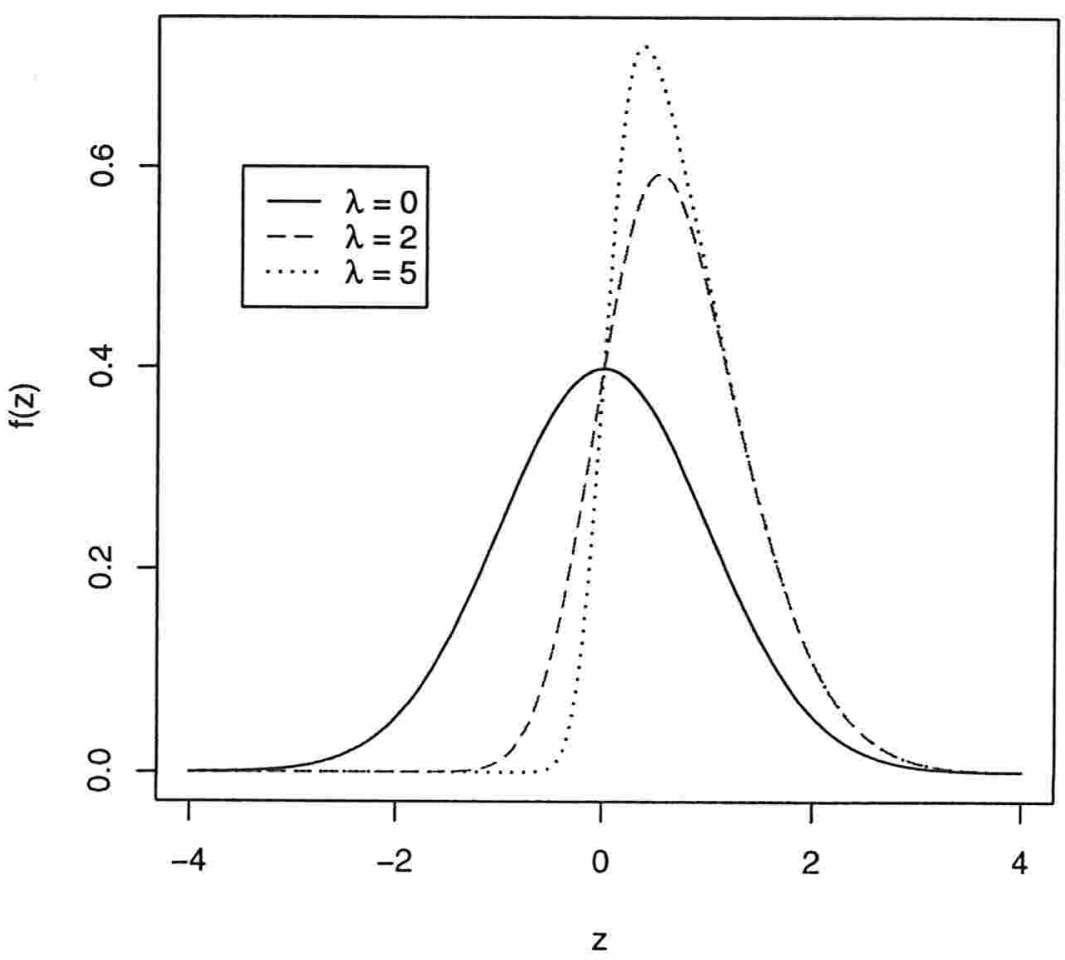


Figura B.2: Funções de densidade da normal assimétrica com valores negativos de $\lambda$

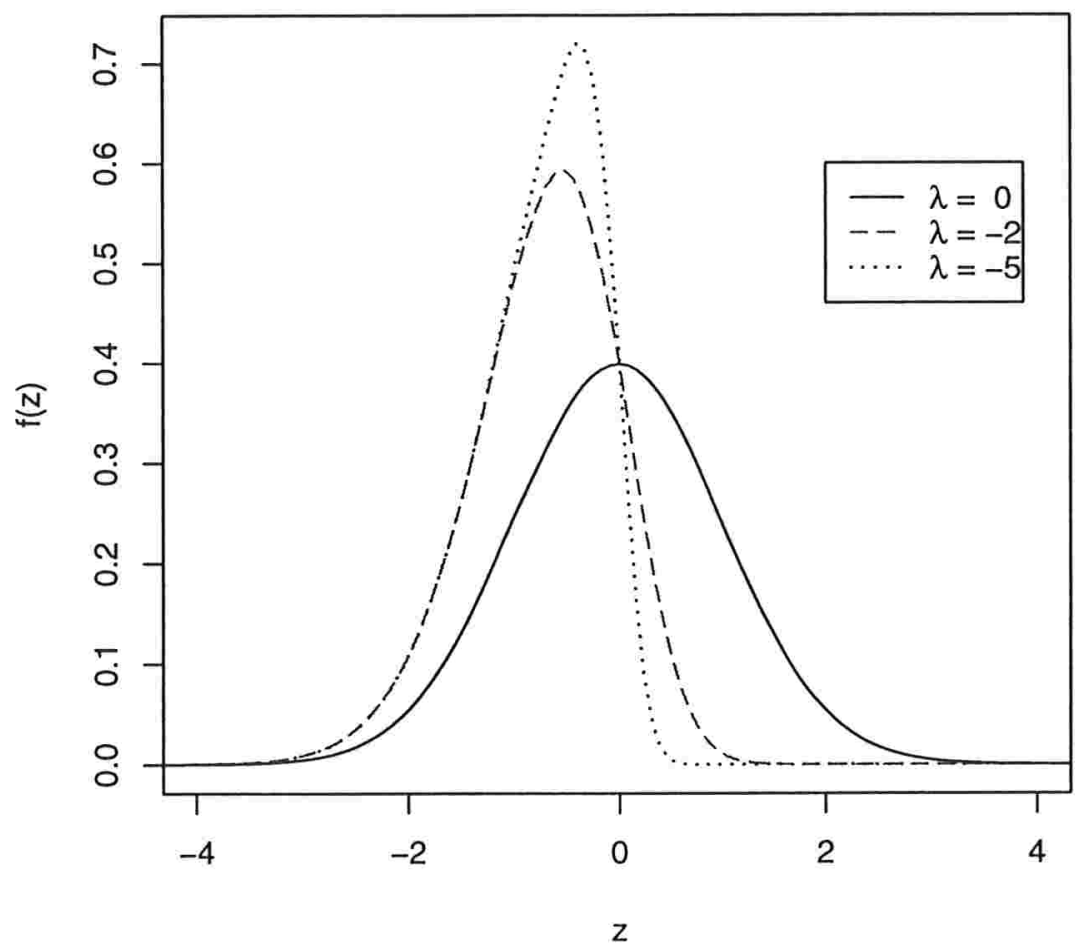


Apêndice $C$

Tabelas 
Tabela C.1: Viés das estimativas no estudo de simulação

\begin{tabular}{|c|c|c|c|c|c|c|c|c|c|}
\hline \multirow[b]{2}{*}{$\mathrm{n}$} & \multirow[b]{2}{*}{$\lambda$} & \multirow[b]{2}{*}{ e.m.v. } & \multicolumn{3}{|c|}{ Priori I : Jeffreys } & \multicolumn{3}{|c|}{ Priori II : Uniforme } & \multirow[b]{2}{*}{$\%$} \\
\hline & & & Máximo & Média & Mediana & Máximo & Média & Mediana & \\
\hline \multirow{7}{*}{30} & 0,5 & 0,05 & 0,01 & 0,04 & 0,03 & $-0,04$ & $-0,01$ & $-0,02$ & 100,00 \\
\hline & 1 & 0,14 & 0,03 & 0,12 & 0,10 & $-0,09$ & 0,00 & $-0,03$ & 99,97 \\
\hline & 2 & 0,57 & 0,07 & 0,52 & 0,60 & $-0,31$ & 0,08 & $-0,07$ & 99,23 \\
\hline & 3 & 1,16 & $-0,01$ & 1,05 & 1,85 & $-0,75$ & 0,15 & $-0,24$ & 96,18 \\
\hline & 5 & 2,38 & $-0,59$ & 2,21 & 5,24 & $-2,07$ & 0,00 & $-1,01$ & 86,26 \\
\hline & 8 & 3,43 & $-2,32$ & 3,20 & 9,59 & $-4,59$ & $-1,09$ & $-2,92$ & 69,95 \\
\hline & 10 & 3,62 & $-3,77$ & 3,77 & 11,59 & $-6,43$ & $-2,22$ & $-4,48$ & 61,98 \\
\hline \multirow{7}{*}{50} & 0,5 & 0,03 & 0,00 & 0,02 & 0,01 & $-0,02$ & $-0,01$ & $-0,01$ & 100,00 \\
\hline & 1 & 0,07 & 0,01 & 0,06 & 0,04 & $-0,05$ & $-0,01$ & $-0,02$ & 100,00 \\
\hline & 2 & 0,27 & 0,05 & 0,23 & 0,19 & $-0,16$ & 0,02 & $-0,04$ & 99,95 \\
\hline & 3 & 0,87 & 0,06 & 0,67 & 0,56 & $-0,39$ & 0,06 & $-0,11$ & 99,73 \\
\hline & 5 & 1,87 & 0,00 & 1,77 & 3,28 & $-1,21$ & 0,29 & $-0,37$ & 95,93 \\
\hline & 8 & 4,45 & $-0,83$ & 3,48 & 8,55 & $-3,18$ & 0,13 & $-1,48$ & 86,53 \\
\hline & 10 & 4,91 & $-1,80$ & 4,25 & 11,69 & $-4,77$ & $-0,40$ & $-2,60$ & 79,86 \\
\hline \multirow{7}{*}{100} & 0,5 & 0,01 & 0,00 & 0,01 & 0,01 & $-0,01$ & 0,00 & $-0,01$ & 100,00 \\
\hline & 1 & 0,03 & 0,01 & 0,03 & 0,02 & $-0,02$ & 0,00 & $-0,01$ & 100,00 \\
\hline & 2 & 0,10 & 0,02 & 0,09 & 0,06 & $-0,08$ & 0,00 & $-0,03$ & 100,00 \\
\hline & 3 & 0,25 & 0,04 & 0,22 & 0,16 & $-0,15$ & 0,02 & $-0,04$ & 100,00 \\
\hline & 5 & 0,87 & 0,13 & 0,79 & 0,72 & $-0,47$ & 0,12 & $-0,09$ & 99,86 \\
\hline & 8 & 2,43 & 0,16 & 2,22 & 3,41 & $-1,45$ & 0,40 & $-0,35$ & 98,26 \\
\hline & 10 & 3,96 & $-0,01$ & 3,53 & 6,75 & $-2,41$ & 0,62 & $-0,71$ & 95,33 \\
\hline \multirow{7}{*}{200} & 0,5 & 0,01 & 0,00 & 0,00 & 0,00 & $-0,01$ & 0,00 & 0,00 & 100,00 \\
\hline & 1 & 0,02 & 0,00 & 0,01 & 0,01 & $-0,01$ & 0,00 & 0,00 & 100,00 \\
\hline & 2 & 0,05 & 0,00 & 0,04 & 0,03 & $-0,04$ & $-0,01$ & $-0,02$ & 100,00 \\
\hline & 3 & 0,10 & 0,02 & 0,09 & 0,07 & $-0,07$ & 0,00 & $-0,02$ & 100,00 \\
\hline & 5 & 0,30 & 0,04 & 0,26 & 0,19 & $-0,21$ & 0,01 & $-0,06$ & 100,00 \\
\hline & 8 & 0,87 & 0,11 & 0,76 & 0,62 & $-0,59$ & 0,07 & $-0,15$ & 99,95 \\
\hline & 10 & 1,67 & 0,23 & 1,46 & 1,47 & $-0,96$ & 0,22 & $-0,20$ & 99,79 \\
\hline
\end{tabular}


Tabela C.2: Erro quadrático médio das estimativas no estudo de simulação

\begin{tabular}{|c|c|c|c|c|c|c|c|c|c|}
\hline & & & \multicolumn{3}{|c|}{ Priori I : Jeffreys } & \multicolumn{3}{|c|}{ Priori II : Uniforme } & \multirow[b]{2}{*}{$\%$} \\
\hline $\mathrm{n}$ & $\lambda$ & e.m.v. & Máximo & Média & Mediana & Máximo & Média & Mediana & \\
\hline \multirow{7}{*}{30} & 0,5 & 0,32 & 0,28 & 0,31 & 0,30 & 0,25 & 0,28 & 0,27 & 100,00 \\
\hline & 1 & 0,57 & 0,43 & 0,55 & 0,72 & 0,35 & 0,44 & 0,40 & 99,97 \\
\hline & 2 & 2,19 & 0,92 & 2,42 & 3,83 & 0,61 & 1,10 & 0,80 & 99,23 \\
\hline & 3 & 4,61 & 1,27 & 4,12 & 8,05 & 0,98 & 1,77 & 1,13 & 96,18 \\
\hline & 5 & 11,27 & 1,80 & 9,62 & 14,64 & 2,18 & 2,58 & 1,73 & 86,26 \\
\hline & 8 & 26,02 & 3,06 & 22,43 & 20,87 & 4,65 & 3,21 & 3,30 & 69,95 \\
\hline & 10 & 18,17 & 4,37 & 23,27 & 23,20 & 6,48 & 3,87 & 4,76 & 61,98 \\
\hline \multirow{7}{*}{50} & 0,5 & 0,22 & 0,21 & 0,22 & 0,22 & 0,20 & 0,20 & 0,20 & 100,00 \\
\hline & 1 & 0,34 & 0,30 & 0,33 & 0,32 & 0,28 & 0,30 & 0,29 & 100,00 \\
\hline & 2 & 0,96 & 0,66 & 0,92 & 1,37 & 0,52 & 0,70 & 0,61 & 99,95 \\
\hline & 3 & 16,80 & 1,14 & 5,78 & 3,93 & 0,81 & 1,32 & 1,01 & 99,73 \\
\hline & 5 & 7,02 & 2,06 & 7,97 & 14,27 & 1,56 & 2,91 & 1,81 & 95,93 \\
\hline & 8 & 79,11 & 2,97 & 17,74 & 24,43 & 3,38 & 4,25 & 2,75 & 86,53 \\
\hline & 10 & 40,59 & 3,60 & 25,00 & 28,90 & 4,92 & 4,66 & 3,57 & 79,86 \\
\hline \multirow{7}{*}{100} & 0,5 & 0,15 & 0,15 & 0,15 & 0,15 & 0,14 & 0,15 & 0,15 & 100,00 \\
\hline & 1 & 0,21 & 0,20 & 0,21 & 0,20 & 0,19 & 0,20 & 0,19 & 100,00 \\
\hline & 2 & 0,45 & 0,40 & 0,45 & 0,43 & 0,38 & 0,40 & 0,39 & 100,00 \\
\hline & 3 & 0,96 & 0,73 & 0,92 & 0,84 & 0,63 & 0,72 & 0,68 & 100,00 \\
\hline & 5 & 3,92 & 1,73 & 3,68 & 6,69 & 1,25 & 1,97 & 1,57 & 99,86 \\
\hline & 8 & 9,95 & 3,36 & 9,42 & 19,38 & 2,25 & 4,39 & 2,88 & 98,26 \\
\hline & 10 & 35,64 & 4,07 & 18,75 & 28,35 & 3,12 & 5,79 & 3,58 & 95,33 \\
\hline \multirow{7}{*}{200} & 0,5 & 0,10 & 0,10 & 0,10 & 0,10 & 0,10 & 0,10 & 0,10 & 100,00 \\
\hline & 1 & 0,15 & 0,14 & 0,14 & 0,14 & 0,14 & 0,14 & 0,14 & 100,00 \\
\hline & 2 & 0,28 & 0,27 & 0,28 & 0,27 & 0,26 & 0,27 & 0,26 & 100,00 \\
\hline & 3 & 0,49 & 0,45 & 0,49 & 0,47 & 0,43 & 0,45 & 0,44 & 100,00 \\
\hline & 5 & 1,14 & 0,98 & 1,12 & 1,07 & 0,89 & 0,97 & 0,94 & 100,00 \\
\hline & 8 & 3,10 & 2,30 & 2,97 & 4,37 & 1,81 & 2,43 & 2,11 & 99,95 \\
\hline & 10 & 11,05 & 3,51 & 7,61 & 13,52 & 2,46 & 4,03 & 3,12 & 99,79 \\
\hline
\end{tabular}


Tabela C.3: Intervalo de confiança assintótico com confiança de $95 \%$

\begin{tabular}{|c|c|c|c|c|c|c|}
\hline $\mathrm{n}$ & $\lambda$ & LI & LS & $0 \in I$ & $\lambda \in I$ & $\%$ \\
\hline \hline \multirow{5}{*}{30} & 0,5 & 0,00 & 1,10 & 50,00 & 95,84 & 100,00 \\
& 1 & 0,33 & 1,96 & 3,86 & 97,08 & 99,97 \\
& 2 & 0,50 & 4,65 & 2,49 & 96,78 & 99,23 \\
& 3 & $-0,11$ & 8,43 & 9,89 & 96,48 & 96,18 \\
& 5 & $-3,38$ & 18,13 & 34,87 & 95,87 & 86,26 \\
& 8 & $-11,92$ & 34,77 & 67,32 & 94,58 & 69,95 \\
& 10 & $-12,42$ & 39,66 & 80,81 & 92,94 & 61,98 \\
\hline \hline \multirow{5}{*}{50} & 0,5 & 0,11 & 0,94 & 25,67 & 95,64 & 100,00 \\
& 1 & 0,49 & 1,65 & 0,08 & 95,96 & 100,00 \\
& 2 & 1,02 & 3,51 & 0,06 & 97,35 & 99,95 \\
& 3 & $-0,89$ & 8,63 & 0,98 & 96,74 & 99,73 \\
& 5 & 0,20 & 13,55 & 8,89 & 96,59 & 95,93 \\
& 8 & $-24,00$ & 48,91 & 29,87 & 95,44 & 86,53 \\
& 10 & $-13,79$ & 43,60 & 45,45 & 95,74 & 79,86 \\
\hline \hline \multirow{5}{*}{100} & 0,5 & 0,23 & 0,80 & 3,46 & 94,93 & 100,00 \\
& 1 & 0,64 & 1,43 & 0,00 & 95,79 & 100,00 \\
& 2 & 1,32 & 2,88 & 0,00 & 96,03 & 100,00 \\
& 3 & 1,88 & 4,62 & 0,01 & 96,68 & 100,00 \\
& 5 & 2,51 & 9,22 & 0,42 & 96,75 & 99,86 \\
& 8 & 1,98 & 18,88 & 3,84 & 96,58 & 98,26 \\
& 10 & $-3,06$ & 30,99 & 8,19 & 95,75 & 95,33 \\
\hline \hline \multirow{5}{*}{0} & 0,5 & 0,30 & 0,71 & 0,00 & 95,24 & 100,00 \\
& 1 & 0,74 & 1,29 & 0,00 & 95,10 & 100,00 \\
& 2 & 1,52 & 2,58 & 0,00 & 95,78 & 100,00 \\
& 3 & 2,21 & 4,00 & 0,00 & 95,78 & 100,00 \\
& 3,40 & 7,76 & 12,98 & 0,04 & 96,73 & 99,95 \\
& 4,94 & 18,40 & 0,38 & 96,66 & 99,79 \\
\hline
\end{tabular}


Tabela C.4: Intervalo de Credibilidade com probabilidade de $95 \%$

\begin{tabular}{|c|c|c|c|c|c|c|c|c|c|}
\hline & & \multicolumn{4}{|c|}{ Priori I : Jeffreys } & \multicolumn{4}{|c|}{ Priori II : Uniforme } \\
\hline $\mathrm{n}$ & $\lambda$ & LI & LS & $0 \in I$ & $\lambda \in I$ & LI & LS & $0 \in I$ & $\lambda \in I$ \\
\hline \multirow{7}{*}{30} & 0,5 & 0,03 & 1,14 & 46,10 & 95,00 & 0,01 & 1,06 & 50,50 & 95,70 \\
\hline & 1,0 & 0,44 & 2,15 & 3,50 & 95,30 & 0,36 & 1,90 & 4,70 & 95,30 \\
\hline & 2,0 & 1,03 & 73,23 & 0,00 & 95,40 & 0,83 & 4,11 & 0,00 & 94,90 \\
\hline & 3,0 & 1,55 & 1006,70 & 0,00 & 91,30 & 1,13 & 7,99 & 0,00 & 93,40 \\
\hline & 5,0 & 2,23 & 2615,92 & 0,00 & 96,00 & 1,46 & 14,41 & 0,00 & 94,20 \\
\hline & 8,0 & 2,88 & 4778,46 & 0,00 & 96,00 & 1,69 & 22,19 & 0,00 & 88,90 \\
\hline & 10,0 & 3,24 & 7151,83 & 0,00 & 97,40 & 1,77 & 27,24 & 0,00 & 90,90 \\
\hline \multirow{7}{*}{50} & 0,5 & 0,12 & 0,94 & 24,80 & 94,50 & 0,11 & 0,91 & 26,70 & 94,70 \\
\hline & 1,0 & 0,54 & 1,70 & 0,20 & 94,60 & 0,50 & 1,61 & 0,30 & 95,30 \\
\hline & 2,0 & 1,19 & 3,63 & 0,00 & 94,00 & 1,06 & 3,29 & 0,00 & 94,20 \\
\hline & 3,0 & 1,75 & 65,27 & 0,00 & 94,50 & 1,50 & 5,62 & 0,00 & 95,30 \\
\hline & 5,0 & 2,71 & 1020,09 & 0,00 & 93,70 & 2,09 & 12,17 & 0,00 & 93,80 \\
\hline & 8,0 & 3,76 & 3687,09 & 0,00 & 96,00 & 2,57 & 22,65 & 0,00 & 92,60 \\
\hline & 10,0 & 4,26 & 5891,26 & 0,00 & 96,30 & 2,75 & 28,69 & 0,00 & 92,10 \\
\hline \multirow{7}{*}{100} & 0,5 & 0,24 & 0,80 & 3,20 & 94,60 & 0,23 & 0,79 & 3,70 & 94,90 \\
\hline & 1,0 & 0,66 & 1,44 & 0,00 & 94,40 & 0,64 & 1,41 & 0,00 & 94,80 \\
\hline & 2,0 & 1,39 & 2,93 & 0,00 & 94,70 & 1,33 & 2,81 & 0,00 & 95,00 \\
\hline & 3,0 & 2,03 & 4,69 & 0,00 & 93,70 & 1,90 & 4,43 & 0,00 & 93,40 \\
\hline & 5,0 & 3,21 & 238,13 & 0,00 & 94,10 & 2,86 & 8,68 & 0,00 & 94,50 \\
\hline & 8,0 & 4,60 & 913,08 & 0,00 & 94,70 & 3,80 & 16,87 & 0,00 & 94,70 \\
\hline & 10,0 & 5,60 & 2882,27 & 0,00 & 91,90 & 4,34 & 25,27 & 0,00 & 93,00 \\
\hline \multirow{7}{*}{200} & 0,5 & 0,30 & 0,70 & 0,00 & 94,50 & 0,30 & 0,69 & 0,00 & 94,30 \\
\hline & 1,0 & 0,75 & 1,29 & 0,00 & 94,00 & 0,74 & 1,28 & 0,00 & 93,70 \\
\hline & 2,0 & 1,55 & 2,60 & 0,00 & 97,00 & 1,52 & 2,55 & 0,00 & 96,50 \\
\hline & 3,0 & 2,29 & 4,07 & 0,00 & 95,00 & 2,22 & 3,96 & 0,00 & 95,60 \\
\hline & 5,0 & 3,59 & 7,30 & 0,00 & 95,30 & 3,41 & 6,98 & 0,00 & 94,60 \\
\hline & 8,0 & 5,38 & 13,50 & 0,00 & 95,50 & 4,94 & 12,47 & 0,00 & 95,10 \\
\hline & 10,0 & 6,50 & 104,99 & 0,00 & 95,00 & 5,83 & 16,93 & 0,00 & 95,70 \\
\hline
\end{tabular}


Tabela C.5: HPD com probabilidade de $95 \%$

\begin{tabular}{|c|c|c|c|c|c|c|c|c|c|}
\hline \multirow[b]{2}{*}{$\mathrm{n}$} & & \multicolumn{4}{|c|}{ Priori I : Jeffreys } & \multicolumn{4}{|c|}{ Priori II : Uniforme } \\
\hline & & LI & LS & $0 \in I$ & $\lambda \in I$ & LI & LS & $0 \in I$ & $\lambda \in I$ \\
\hline \multirow{7}{*}{30} & 0,5 & 0,02 & 1,11 & 49,00 & 95,60 & $-0,01$ & 1,04 & 54,00 & 96,50 \\
\hline & 1,0 & 0,39 & 2,05 & 4,00 & 96,00 & 0,32 & 1,82 & 6,00 & 96,30 \\
\hline & 2,0 & 0,86 & 21,60 & 0,00 & 97,10 & 0,69 & 3,76 & 0,00 & 94,10 \\
\hline & 3,0 & 1,09 & 257,46 & 0,00 & 95,90 & 0,86 & 6,70 & 0,00 & 90,60 \\
\hline & 5,0 & 1,26 & 664,20 & 0,00 & 96,80 & 0,99 & 11,41 & 0,00 & 90,10 \\
\hline & 8,0 & 1,26 & 1209,19 & 0,00 & 94,40 & 1,01 & 16,84 & 0,00 & 83,40 \\
\hline & 10,0 & 1,17 & 1804,99 & 0,00 & 96,30 & 0,99 & 20,16 & 0,00 & 84,50 \\
\hline \multirow{7}{*}{50} & 0,5 & 0,11 & 0,93 & 25,80 & 94,80 & 0,10 & 0,89 & 27,40 & 95,00 \\
\hline & 1,0 & 0,52 & 1,67 & 0,20 & 95,50 & 0,47 & 1,58 & 0,30 & 95,30 \\
\hline & 2,0 & 1,10 & 3,48 & 0,00 & 95,40 & 0,97 & 3,15 & 0,00 & 93,90 \\
\hline & 3,0 & 1,54 & 21,04 & 0,00 & 96,50 & 1,32 & 5,20 & 0,00 & 93,50 \\
\hline & 5,0 & 2,06 & 264,33 & 0,00 & 96,10 & 1,68 & 10,41 & 0,00 & 91,20 \\
\hline & 8,0 & 2,31 & 936,63 & 0,00 & 96,80 & 1,84 & 18,07 & 0,00 & 88,60 \\
\hline & 10,0 & 2,33 & 1492,75 & 0,00 & 95,60 & 1,87 & 22,26 & 0,00 & 87,60 \\
\hline \multirow{7}{*}{100} & 0,5 & 0,23 & 0,80 & 3,60 & 95,10 & 0,22 & 0,78 & 4,00 & 95,00 \\
\hline & 1,0 & 0,65 & 1,43 & 0,00 & 95,10 & 0,62 & 1,39 & 0,00 & 94,30 \\
\hline & 2,0 & 1,35 & 2,88 & 0,00 & 95,40 & 1,28 & 2,76 & 0,00 & 94,20 \\
\hline & 3,0 & 1,94 & 4,57 & 0,00 & 93,80 & 1,81 & 4,30 & 0,00 & 92,80 \\
\hline & 5,0 & 2,93 & 66,26 & 0,00 & 96,20 & 2,61 & 8,16 & 0,00 & 93,70 \\
\hline & 8,0 & 3,82 & 243,03 & 0,00 & 96,40 & 3,25 & 14,90 & 0,00 & 91,70 \\
\hline & 10,0 & 4,24 & 737,98 & 0,00 & 95,20 & 3,52 & 21,32 & 0,00 & 89,60 \\
\hline \multirow{7}{*}{200} & 0,5 & 0,30 & 0,70 & 0,00 & 94,60 & 0,30 & 0,69 & 0,00 & 94,30 \\
\hline & 1,0 & 0,75 & 1,29 & 0,00 & 94,00 & 0,73 & 1,27 & 0,00 & 93,50 \\
\hline & 2,0 & 1,53 & 2,58 & 0,00 & 97,00 & 1,50 & 2,52 & 0,00 & 96,80 \\
\hline & 3,0 & 2,24 & 4,02 & 0,00 & 95,40 & 2,17 & 3,90 & 0,00 & 95,10 \\
\hline & 5,0 & 3,47 & 7,14 & 0,00 & 95,20 & 3,30 & 6,82 & 0,00 & 94,20 \\
\hline & 8,0 & 5,06 & 13,00 & 0,00 & 95,60 & 4,64 & 11,98 & 0,00 & 95,10 \\
\hline & 10,0 & 5,98 & 40,07 & 0,00 & 96,60 & 5,36 & 15,98 & 0,00 & 94,50 \\
\hline
\end{tabular}


Tabela C.6: Porcentagem de aceitação da hipótese nula com o Teste da Razão de Verossimilhanças

\begin{tabular}{|c|c|c|c|}
\hline $\mathrm{n}$ & $\lambda$ & $H_{0}: \lambda=0$ & $H_{0}: \lambda=\lambda^{*}$ \\
\hline \hline \multirow{5}{*}{30} & 0 & 94,19 & 94,19 \\
& 0,1 & 92,13 & 94,41 \\
& 0,25 & 80,42 & 94,80 \\
& 0,3 & 73,80 & 94,54 \\
& 0,5 & 44,88 & 93,84 \\
& 0,75 & 16,24 & 94,18 \\
& 1 & 3,95 & 93,89 \\
\hline \hline \multirow{5}{*}{50} & 0 & 94,50 & 94,50 \\
& 0,1 & 91,45 & 94,82 \\
& 0,25 & 72,10 & 94,28 \\
& 0,3 & 61,47 & 94,45 \\
& 0,5 & 23,14 & 94,91 \\
& 0,75 & 3,39 & 94,58 \\
& 1 & 0,23 & 94,72 \\
\hline \hline \multirow{51}{*}{100} & 0 & 94,96 & 94,96 \\
& 0,1 & 86,61 & 94,76 \\
& 0,25 & 51,07 & 95,02 \\
& 0,3 & 35,71 & 94,68 \\
& 0,5 & 3,10 & 94,57 \\
& 0,75 & 0,00 & 94,95 \\
& 1 & 0,00 & 94,88 \\
\hline \hline \multirow{5}{*}{0} & 0 & 94,76 & 94,76 \\
& 0,1 & 79,59 & 94,83 \\
& 0,25 & 21,04 & 94,70 \\
& 0,3 & 8,83 & 94,86 \\
& 0,5 & 0,02 & 94,97 \\
& 0,00 & 94,64 \\
& 0,00 & 94,49 \\
\hline
\end{tabular}


Tabela C.7: Porcentagem de aceitação (ou rejeição) da hipótese nula $H_{0}: \lambda=0$, com o Fator de Bayes

\begin{tabular}{|c|c|c|c|c|c|}
\hline \multirow[b]{2}{*}{$\mathrm{n}$} & \multirow[b]{2}{*}{$\lambda$} & \multicolumn{2}{|c|}{ Priori I : Jeffreys } & \multicolumn{2}{|c|}{ Priori II : Uniforme } \\
\hline & & $B F_{0}>2$ & $B F_{0}<0.5$ & $B F_{0}>2$ & $B F_{0}<0.5$ \\
\hline \multirow{7}{*}{30} & 0,00 & 92,33 & 1,52 & 74,30 & 3,93 \\
\hline & 0,10 & 89,49 & 2,76 & 68,38 & 5,78 \\
\hline & 0,25 & 76,26 & 8,06 & 50,40 & 15,90 \\
\hline & 0,30 & 69,12 & 11,90 & 42,38 & 21,52 \\
\hline & 0,50 & 38,61 & 33,74 & 16,42 & 49,73 \\
\hline & 0,75 & 11,71 & 67,74 & 2,85 & 80,67 \\
\hline & 1,00 & 2,39 & 89,30 & 0,29 & 95,26 \\
\hline \multirow{7}{*}{50} & 0,00 & 94,17 & 1,22 & 80,75 & 3,74 \\
\hline & 0,10 & 90,89 & 2,45 & 74,62 & 5,76 \\
\hline & 0,25 & 70,65 & 12,08 & 46,59 & 21,83 \\
\hline & 0,30 & 60,61 & 18,32 & 36,59 & 30,97 \\
\hline & 0,50 & 23,51 & 53,27 & 8,25 & 68,75 \\
\hline & 0,75 & 2,64 & 89,68 & 0,48 & 95,54 \\
\hline & 1,00 & 0,15 & 98,84 & 0,00 & 99,69 \\
\hline \multirow{7}{*}{100} & 0,00 & 96,43 & 0,74 & 87,71 & 2,26 \\
\hline & 0,10 & 90,61 & 3,07 & 76,66 & 6,68 \\
\hline & 0,25 & 56,55 & 23,04 & 34,45 & 36,59 \\
\hline & 0,30 & 41,10 & 35,90 & 21,36 & 51,74 \\
\hline & 0,50 & 4,84 & 85,76 & 1,17 & 92,98 \\
\hline & 0,75 & 0,06 & 99,70 & 0,00 & 99,93 \\
\hline & 1,00 & 0,00 & 100,00 & 0,00 & 100,00 \\
\hline \multirow{7}{*}{200} & 0,00 & 97,77 & 0,47 & 91,50 & 1,52 \\
\hline & 0,10 & 87,28 & 4,64 & 73,26 & 9,80 \\
\hline & 0,25 & 30,83 & 49,01 & 14,96 & 64,17 \\
\hline & 0,30 & 14,44 & 69,02 & 5,56 & 81,94 \\
\hline & 0,50 & 0,05 & 99,56 & 0,00 & 99,93 \\
\hline & 0,75 & 0,00 & 100,00 & 0,00 & 100,00 \\
\hline & 1,00 & 0,00 & 100,00 & 0,00 & 100,00 \\
\hline
\end{tabular}


Tabela C.8: Porcentagem de aceitação (ou rejeição) da hipótese nula $H_{0}: \lambda=\lambda^{*}$, com o Fator de Bayes

\begin{tabular}{|c|c|c|c|c|c|}
\hline \multirow[b]{2}{*}{$\mathrm{n}$} & \multirow[b]{2}{*}{$\lambda$} & \multicolumn{2}{|c|}{ Priori I : Jeffreys } & \multicolumn{2}{|c|}{ Priori II : Uniforme } \\
\hline & & $B F>2$ & $B F<0.5$ & $B F>2$ & $B F<0.5$ \\
\hline \multirow{7}{*}{30} & 0,00 & 92,33 & 1,52 & 74,30 & 3,93 \\
\hline & 0,10 & 92,03 & 1,73 & 73,29 & 4,24 \\
\hline & 0,25 & 91,77 & 1,97 & 75,60 & 4,49 \\
\hline & 0,30 & 92,62 & 1,61 & 76,54 & 3,72 \\
\hline & 0,50 & 92,25 & 1,47 & 79,93 & 3,36 \\
\hline & 0,75 & 92,20 & 1,74 & 84,10 & 2,89 \\
\hline & 1,00 & 91,84 & 1,84 & 87,77 & 2,30 \\
\hline \multirow{7}{*}{50} & 0,00 & 94,17 & 1,22 & 80,75 & 3,74 \\
\hline & 0,10 & 94,40 & 1,23 & 81,28 & 3,26 \\
\hline & 0,25 & 94,40 & 0,99 & 82,14 & 3,13 \\
\hline & 0,30 & 94,54 & 1,17 & 82,69 & 2,94 \\
\hline & 0,50 & 94,74 & 1,07 & 85,50 & 2,55 \\
\hline & 0,75 & 94,23 & 1,21 & 88,58 & 2,21 \\
\hline & 1,00 & 94,25 & 1,22 & 90,40 & 1,75 \\
\hline \multirow{7}{*}{100} & 0,00 & 96,43 & 0,74 & 87,71 & 2,26 \\
\hline & 0,10 & 96,26 & 0,84 & 88,28 & 2,35 \\
\hline & 0,25 & 96,11 & 0,70 & 88,51 & 2,39 \\
\hline & 0,30 & 96,28 & 0,90 & 88,41 & 2,21 \\
\hline & 0,50 & 96,08 & 0,82 & 90,02 & 2,10 \\
\hline & 0,75 & 96,54 & 0,81 & 92,69 & 1,60 \\
\hline & 1,00 & 96,53 & 0,75 & 94,20 & 1,16 \\
\hline \multirow{7}{*}{200} & 0,00 & 97,77 & 0,47 & 91,50 & 1,52 \\
\hline & 0,10 & 97,65 & 0,44 & 92,11 & 1,47 \\
\hline & 0,25 & 97,56 & 0,52 & 92,21 & 1,48 \\
\hline & 0,30 & 97,70 & 0,55 & 92,49 & 1,48 \\
\hline & 0,50 & 97,72 & 0,35 & 93,90 & 1,21 \\
\hline & 0,75 & 97,54 & 0,52 & 94,83 & 1,00 \\
\hline & 1,00 & 97,12 & 0,50 & 95,63 & 0,87 \\
\hline
\end{tabular}




\section{Apêndice D}

\section{Programas}

\section{D.1 Programa em WinBUGS para o modelo de três parâmetros}

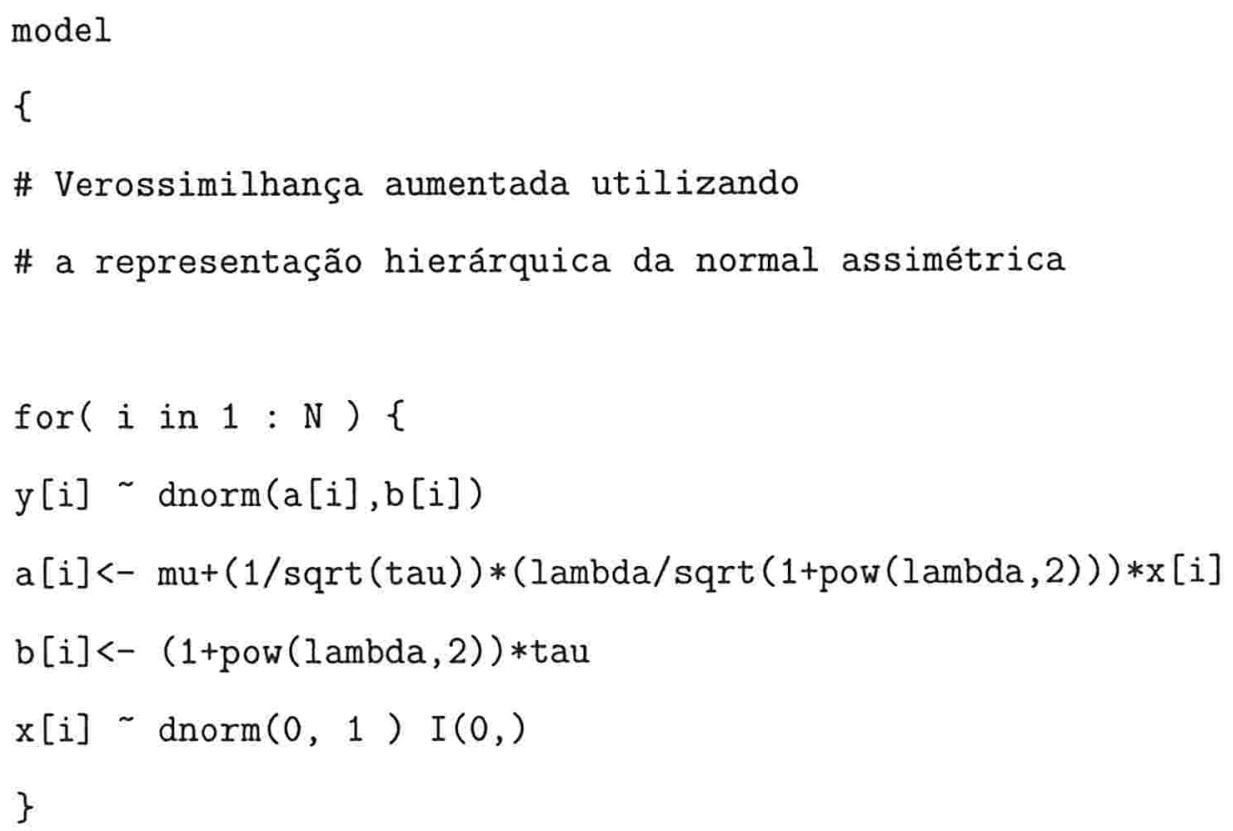


\# Especificação a priori para os parâmetros de posição e escala

$\mathrm{mu} \sim \operatorname{dnorm}(0.0,0.01)$

tau $\operatorname{dgamma}(0.01,0.01)$

\# Especificação a priori para o parâmetro de assimetria

\# Priori I : Aproximação para a priori de Jeffreys

lambda $\operatorname{dnorm}(0$, ww)

wW $<-0.4052847 * \mathrm{w}$

w $\sim \operatorname{dgamma}(0.25,0.25)$

\# Priori II : Uniforme

\# lambda $\operatorname{dnorm}(0, w w)$

\# $\mathrm{wW}<-2 * \mathrm{w}$

\# $\mathrm{w} \sim \operatorname{dgamma}(1,1)$

\}

\section{D.2 Programas em $\mathrm{R}$}

Nesta seção apresentamos os programas em $R$ para a implementação do algoritmo de Gibbs para o modelo de três parâmetros e para o modelo de regressão linear

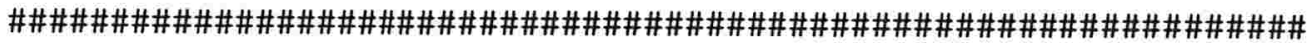
\#\#\#\#\#\#\#\#\#\#\#\#\#\# Amostrador de Gibbs \#\#\#\#\#\#\#\#\#\#\#\#\#\#\#\#\#\#\#\#\#\#

library(mvtnorm) 
\#\#\#\#\#\#\#\# Função para mudar da parametrização \#\#\#\#\#\#\#\#\#\#\#\#\# \#\#\#\#\#\#\#\#\# direta á parametrização nova \#\#\#\#\#\#\#\#\#\#\#\#\#\#\#\#\#\#\#

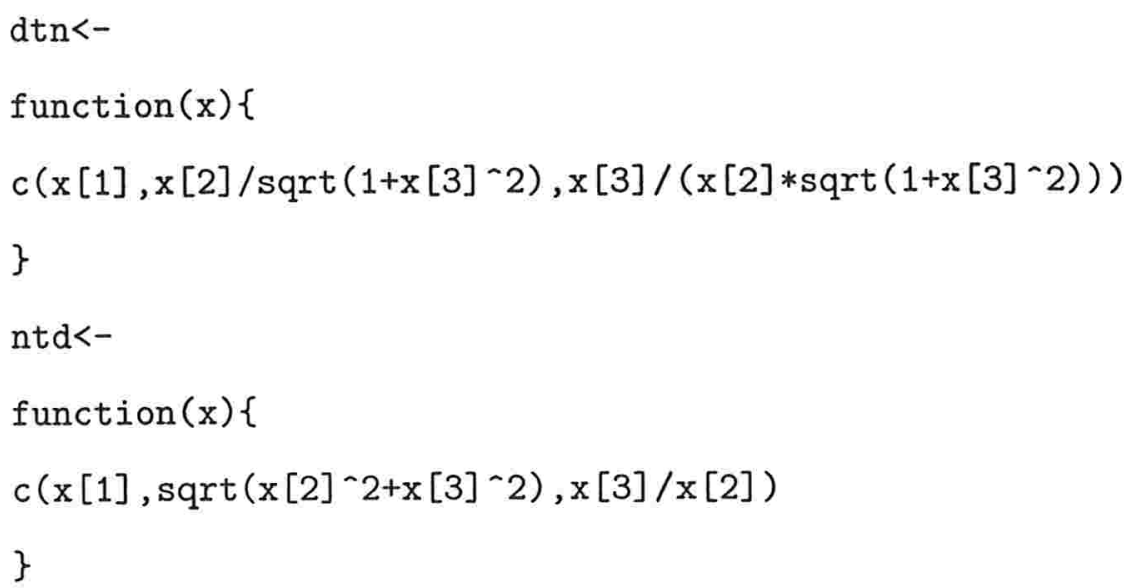

\#\#\# Função para gerar da cauda de uma distribuição normal \#\#\#\# \#\# Metodo de Marsaglia-Non-Uniform Random Variate Generation \#\# \#\#\#\#\#\#\#\# Luc Devroye Capítulo 9 - pag 380-381 \#\#\#\#\#\#\#\#\#\#\#\#

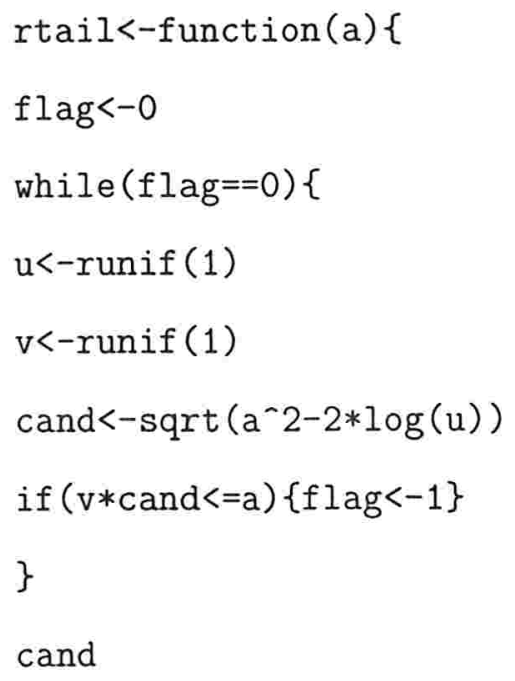


\}

\#\#\#\#\#\#\#\# Função para gerar de uma normal truncada \#\#\#\#\#\#\#\#\#\#\#

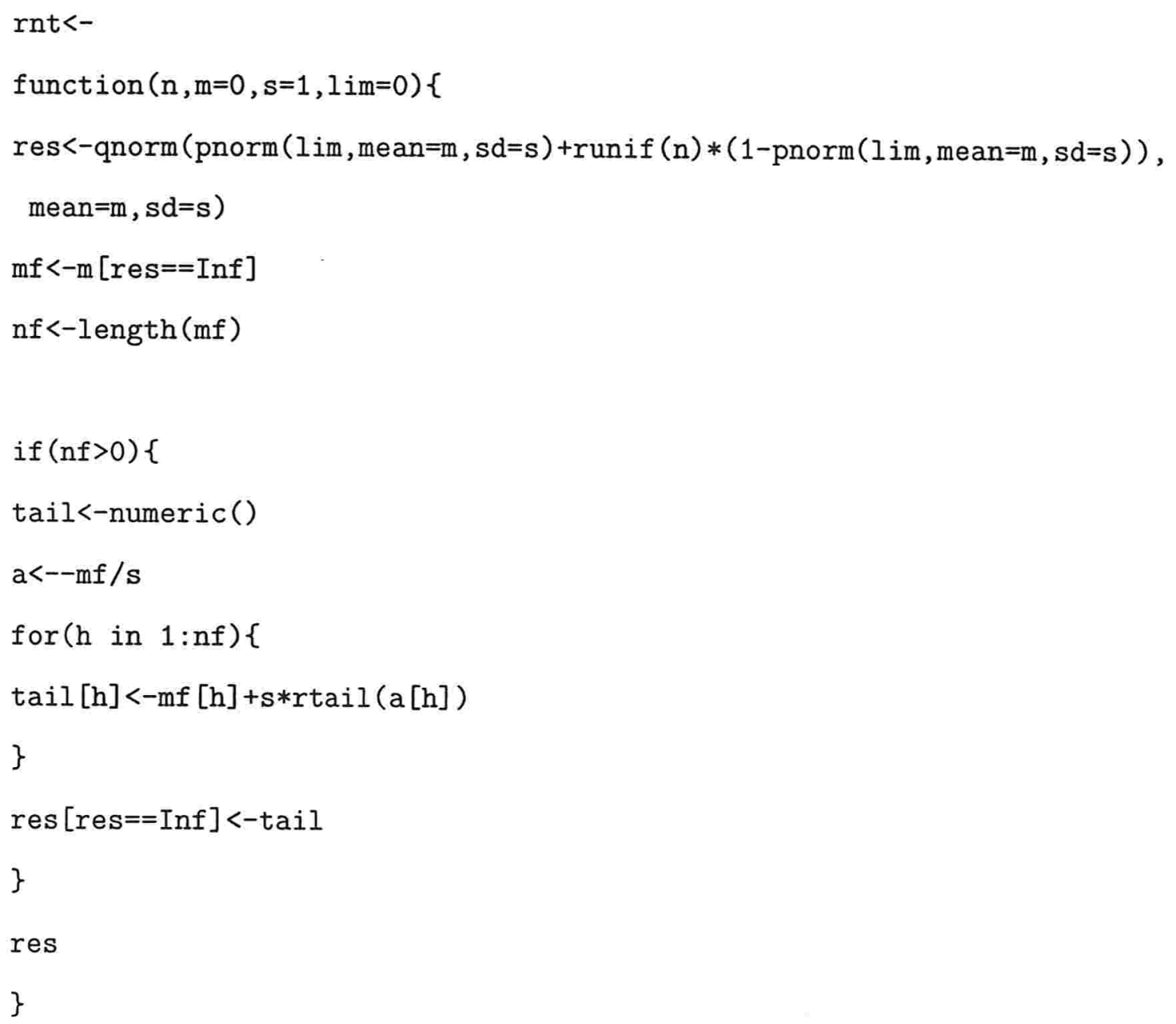

\#\#\#\#\#\#\#\# Função que implementa o Algoritmo de Gibbs \#\#\#\#\#\#\#\#\# \#\#\#\#\#\#\#\#\# para o modelo de três parâmetros \#\#\#\#\#\#\#\#\#\#\#\#\#\#\#\#\# 
gibbs $3<-f u n c t i o n(z, i n i=d \operatorname{tn}(c(0,1,5)), n s i m=10000$, sigma.t, gl.t $)\{$

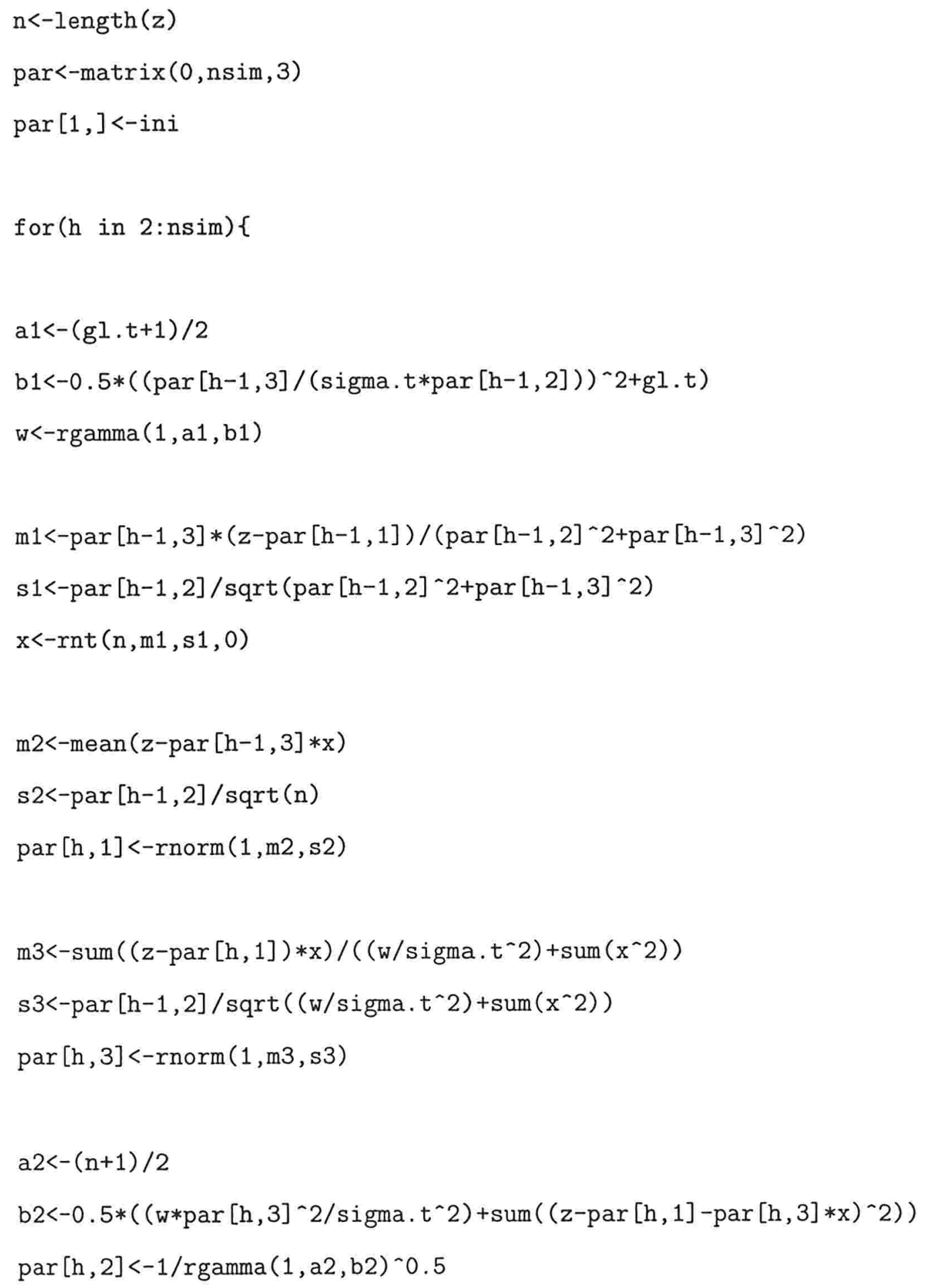


\}

par

\}

\#\#\#\#\#\#\#\# Função que implementa o Algoritmo de Gibbs \#\#\#\#\#\#\#\#\# \#\#\#\#\#\#\#\# para o modelo de regressão linear simples \#\#\#\#\#\#\#\#\#\#

gibbsR<-

function $(Y, X, \operatorname{ini}=\operatorname{dtn}(c(1,1,1,5)), n s i m=10000$, sigma.t,gl.t $)\{$

$\mathrm{n}<-$ length $(\mathrm{Y})$

$\mathrm{p}<-\mathrm{ncol}(\mathrm{X})$

par<-matrix $(0$, nsim, $p+2)$

$\operatorname{par}[1]<$,- ini

for (h in $2: \mathrm{nsim})\{$

$\mathrm{a} 1<-(\mathrm{gl} \cdot \mathrm{t}+1) / 2$

$\mathrm{b} 1<-0.5 *((\operatorname{par}[\mathrm{h}-1, \mathrm{p}+2] /(\operatorname{sigma} . t * \operatorname{par}[\mathrm{h}-1, \mathrm{p}+1])) \wedge 2+\mathrm{gl} . \mathrm{t})$

$\mathrm{w}<-\operatorname{rgamma}(1, \mathrm{a} 1, \mathrm{~b} 1)$

$\mathrm{m} 1<-\mathrm{c}\left(\operatorname{par}[\mathrm{h}-1, \mathrm{p}+2] *(\mathrm{Y}-\mathrm{X} \% * \% \operatorname{par}[\mathrm{h}-1,1: \mathrm{p}]) /\left(\operatorname{par}[\mathrm{h}-1, \mathrm{p}+1]^{\wedge} 2+\operatorname{par}[\mathrm{h}-1, \mathrm{p}+2]^{\wedge} 2\right)\right)$

$\mathrm{s} 1<-\operatorname{par}[\mathrm{h}-1, \mathrm{p}+1] / \operatorname{sqrt}\left(\operatorname{par}[\mathrm{h}-1, \mathrm{p}+1]^{\wedge} 2+\operatorname{par}[\mathrm{h}-1, \mathrm{p}+2]^{\wedge} 2\right)$ 


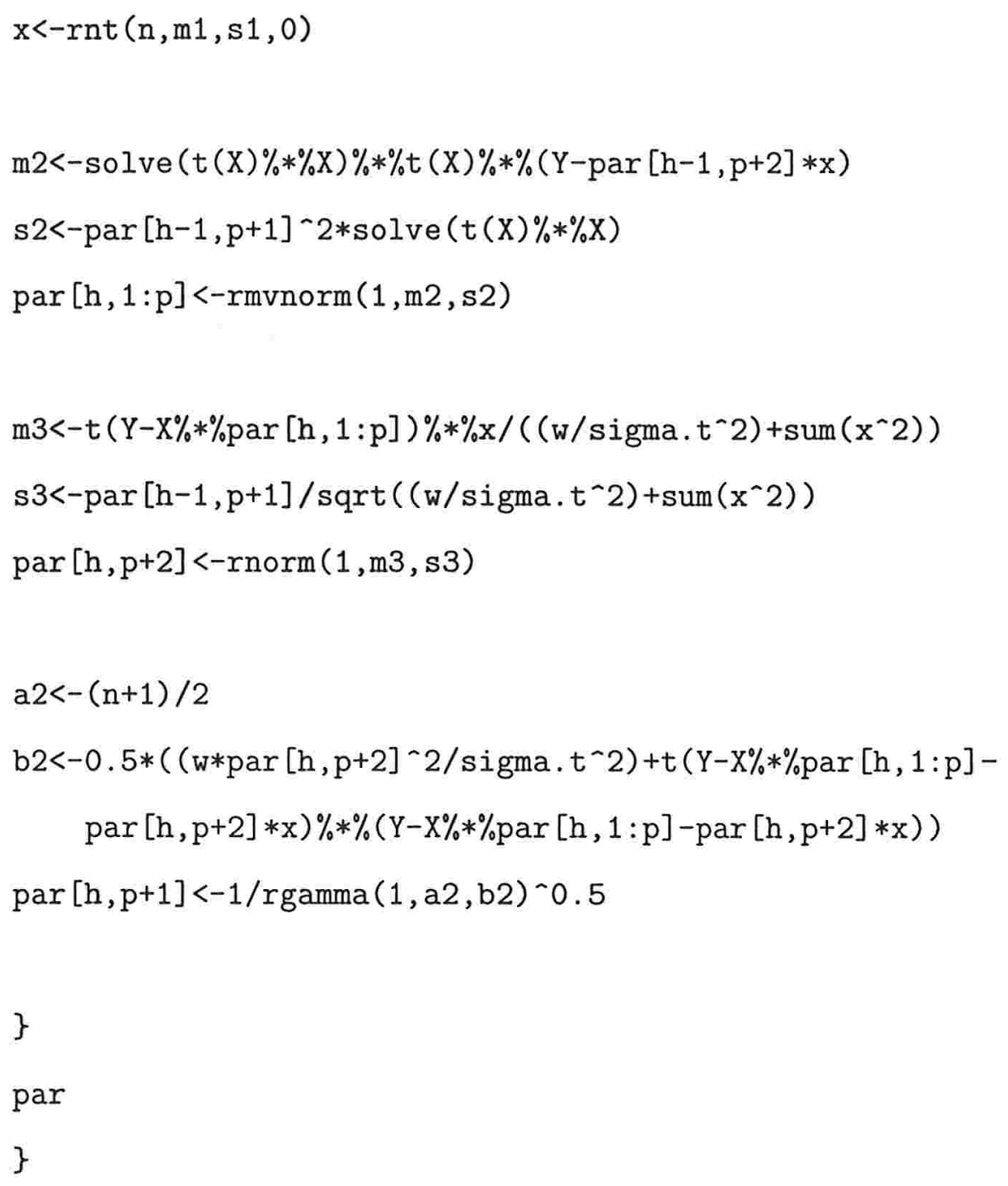




\section{Referências Bibliográficas}

Aigner, D., Lovell, C. \& Schmidt, P. (1977). Formulation and estimation of stochastic frontier production function model, Journal of Econometrics 12: 12-37.

Azzalini, A. (1985). A class of distributions wich includes the normal ones, Scandinavian Journal of Statistics 12: 171-178.

Azzalini, A. (2004). The skew-normal distribution and related multivariate families. http://tango.stat.unipd.it/SN/review.ps.

Azzalini, A. \& Capitanio, A. (1999). Statistical applications of the multivariate skew-normal distribution, Journal of the Royal Statistical Society: Series B 61(3): 579-602.

Azzalini, A. \& Dalla Valle, A. (1996). The multivariate skew-normal distribution, Biometrika 83: 715-726.

Berger, J. \& Bernardo, J. (1992). On the development of reference priors, Bayesian Statistics 4: 35-60.

Chaibub Neto, E. \& Branco, M. (2003). Bayesian reference analysis for binomial calibration problem, RT-MAE 2003-12: IME -USP.

Chen, M., Shao, Q. \& Ibrahim, J. (2001). Monte Carlo Methods in Bayesian Computation, Springer. 
Fang, K. T., Kotz, S. \& Ng, K. W. (1990). Symmetric Multivariate and Related Distributions, Chapman and Hall.

Firth, D. (1993). Bias reduction of maximum likelihood estimates, Biometrika 82: 2738.

Genton, M., He, L. \& Liu, X. (2001). Moments of skew-normal random vectors and their quadratic forms, Statistics and Probability Letters 51: 319-325.

Henze, N. (1986). A probabilistic representation of the skew-normal distribution, Scandinavian Journal of Statistics 13: 271-275.

Johnson, N., Kotz, S. \& Balakrishnan, N. (1994). Continuous Univariate Distributions, Vol. 1, Wiley.

Lachos, V. (2004). Modelos Lineares Mistos Assimétricos, PhD thesis, IME-USP.

Liseo, B. \& Loperfido, N. (2004). A note on reference priors for the scalar skew-normal distribution. Journal of Statistical Planning and Inference to appear.

Liu, C. (2003a). Alternating subspace spanning resampling to accelarate Markov Chain Monte Carlo simulation, Journal of the American Statistical Society 98: $110-117$.

Liu, C. (2003b). An example of algorithm mining: covariance adjustment to accelarate EM and Gibbs, Development of Modern Statistics and Related Topics. edited by Heping Zhang and Jian Huang.

Liu, C., Rubin, D. \& Wu, Y. (1998). Parameter expansion to accelarate EM : the PX-EM algorithm, Biometrika 85: 755-770.

Liu, J. \& Wu, Y. (1999). Parameter expansion for data augmentation, Journal of the American Statistical Society 94: 247-265. 
O'Hagan, A. \& Leonard, T. (1976). Bayes estimation subject to uncertainty about parameter constraints, Biometrika 63: 201-202.

Owen, D. (1980). A table of normal integrals, Communications in Statistics : Simulation and Computation 9(4): 389-419.

Paulino, C., Turkman, M. \& Murteira, B. (2003). Estatística Bayesiana, Fundação Calouste Gulbenkian, Lisboa.

Pewsey, A. (2000). Problems of inference for Azzalini's skew-normal distribution, Journal of Applied Statistics 27: 859-870.

Roberts, C. (1966). A correlation model useful in the study of twins, Journal of the American Statistical Society 61: 1184-1190.

Sahu, S., Dey, D. \& Branco, M. (2003). A new class of multivariate skew distributions with aplications to bayesian regression models, The Canadian Journal of Statistics 31(2): 129-150.

Sartori, N. (2003). Bias prevention of maximum likelihood estimates: skew normal and skew $t$ distributions, Technical report, Dip. Scienze Statistiche, Università di Padova, Italy.

Vidal, I., Iglesias, P., Branco, M. \& Arellano-Valle, R. (2004). Bayesian sensitivity analysis and model comparison for skew elliptical models. Journal of Statistical Planning and Inference to appear. 